\title{
Evolution of cosmological perturbations in the universe dominated by resonant scalar fields
}

\author{
Takashi Hamazaki ${ }^{1}$ \\ Kamiyugi 3-3-4-606 Hachioji-city \\ Tokyo 192-0373 Japan
}

\begin{abstract}
Recently a Hamiltonian formulation for the evolution of the universe dominated by multiple oscillatory scalar fields was developed by the present author and was applied to the investigation of the evolution of cosmological perturbations on superhorizon scales in the case that scalar fields have incommensurable masses.

In the present paper, the analysis is extended to the case in which the masses of scalar fields satisfy resonance conditions approximately. In this case, the action-angle variables for the system can be classified into fast changing variables and slowly changing variables. We show that after an appropriate canonical transformation, the part of the Hamiltonian that depends on the fast changing angle variables can be made negligibly small, so that the dynamics of the system can be effectively determined by a truncated Hamiltonian that describes a closed dynamics of the slowly changing variables. Utilizing this formulation, we show that the system is unstable if this truncated Hamiltonian system has hyperbolic fixed point and as a consequence, the Bardeen parameter for a perturbation of the sytem grows.

PACS number(s):98.80.Cq
\end{abstract}

\footnotetext{
${ }^{1}$ email address: yj4t-hmzk@asahi-net.or.jp
} 


\section{$\S 1 \quad$ Introduction and Summary}

The inflationary universe model is the most successful model in explaining the origin of the present cosmological structures such as galaxies and clusters of galaxies. In this model, quantum fluctuations of an inflaton field, a scalar field driving the inflationary expansion, provide seed perturbations which grow and form the present cosmological structures by gravitational instability. During the slow rolling phase of inflation, these seed perturbations are streched beyond the Hubble horizon and their wavelengths stay larger than the horizon scale until the perturbations come back inside the Hubble horizon during the Friedmann stage after the inflation. The amplitudes of perturbations at this second horizon crossing, which have a direct relevance to the CMB anisotropy observations and provide the initial condition for detailed astrophysical models of galaxy formation, are determined by the so-called Bardeen parameter. Hence, in order to obtain information on the inflationary stage of the universe from observations of the present universe, we have to determine the behavior of the Bardeen parameter of perturbations during the superhorizon stage.

If the cosmic matter has a regular equation of state and is dominated by a single component, this Bardeen parameter is conserved with a good accuracy on superhorizon scales [2]. However, in a realistic model, during the period between the first horizon crossing in the inflationary regime and the socond horizon crossing in the Friedmann regime, the inflaton field oscillates coherently around a local minimum of a potential and their energy is gradually transformed into matter and radiation which constitute the present universe. During this reheating phase, the equation of state becomes singular periodically [6], and entropy modes can be produced [7, 11]. Further, in an inflationary model with a multiplecomponent inflaton field, isocurvature modes can appear [11, 12]. Therefore, the Bardeen parameter may not be converved even approximately in realistic models, and a detailed analysis of its behavior is mandatory.

On superhorizon scales, this problem can be reduced to the analysis of a spatially homogeneous model for the following reasons. First, the evolution of cosmological perturbations on superhorizon scales is well described by that in the long wavelength limit with a good accuracy [6] ]. Further, the long wavelength limit of a solution to the perturbation equation can be easily constructed from a homogeneous perturbation of the background universe model 3, 4, 5] ]. Hence, we only have to analyse a spatially homogeneous system, which is much simpler than the analysis of the exact perturbation equation with a finite wave number. If the background system is exactly solvable, this reduction solves the problem completely. However, in the case of multiple scalar fields in an expanding universe, it is not the case. Further, the system exhibits quite complicated oscillatory behavior.

There is, however, one powerful method to treat such an oscillatory system. It is the Hamiltonian formulation in terms of the action-angle variables [15]. In fact, in a previous paper by the present author [8] ], we have developed a Hamiltonian formulation for a universe model dominated by multiple osillatory scalar fields and have shown that it works well at least in the case in which the scalar fields have incommensurable masses. In this formulation, we introduce an expansion parameter $\epsilon$ that represents the ratio of the cosmic expansion rate to the masses of the scalar fields, and decompose the Hamiltonian is into an unperturbed part that depends only on the action variables and a perturbative part that is of the order $\epsilon$ and bounded by a constant multiple of $1 / t$, where $t$ is a time parameter of the system given by $a^{3 / 2}$ in terms of the cosmic scale factor $a$. Next, we look 
for a canonical transformation that transforms the perturbative part to a quantity of higher order with respect to $\epsilon$ and $1 / t$. As was shown in Ref. [8], we can construct such a canonical transformation iteratively, and by repeated applications of such transformations, we can transform the perturbative part to a quantity of the order of an arbitrary power of $\epsilon$ and $1 / t$. Furthermore, the new Hamiltonian system obtained by these transformations becomes solvable if the small perturbative part is neglected. By this method, we have proved that the Bardeen parameter is conserved with a good accuracy under the assumption that the masses of the scalar fields do not satisfy resonant relations.

In the reheating phase, a dynamical instability caused by the parametric resonance plays a crucial role in the energy transfer from a macroscopic homogeneous mode to finite wavelength modes [9]. This instability can have a significant effect on the evolution of cosmological perturbations [10, 13] ]. In fact, a numerical example showing a non-conservation of the Bardeen parameter was presented in [11]. In order to treat this problem, it is necessary to extend our Hamiltonian formulation to the resonant case. From this point of view, in this paper, we undertake this extension and with the help of it, we investigate the dynamical behavior of the universe dominated by multiple oscillatory scalar fields whose masses satisfy a resonance condition at least approximately.

The present paper is organized as follows. First, in the next section, on the basis of the paper [4], we explain how to construct a solution to the perturbation equation in the long wavelength limit from an exactly spatially homogeneous perturbation of the background universe model. Then, in $\S 3$, we put the spatially homogeneous system of multiple scalar fields in an expanding universe into the Hamiltonian form and introduce the action-angle variables.

In $\S 4$, for the case in which the masses of scalar fields satisfy resonance conditions approximately, we decompose the action-angle variables to fast changing variables and slowly changing variables, and reduce the dynamics of the system to that of slowly changing variables by a canonical transformation. Then, with the help of this formulation, we estimate the growth rate of perturbations of the system in the case in which the time parameter $t$ of the system is smaller than $1 / \epsilon$. On the basis of this estimate and the analysis of some soluble examples, we argue that the Bardeen parameter of the system can grow if the system has a hyperbolic fixed point. Next, in $\S 5$, we analyze the evolution of a perturbation of the system in the time range $t>1 / \epsilon$ and show that the Bardeen parameter is conserved with a good accuracy in this time range. $\$ 6$ is devoted to discussions. In order to make the presentation clear, the proofs of most of the mathematical statements and the calculation of the growth rates of perturbations in concrete models are given the Appendices.

Throughout the paper, the natural units $c=\hbar=1$ are adopted, and $8 \pi G$ is denoted as $\kappa^{2}$. Further, the notation adopted in the article[1] is used for perturbation variables, and their definitions are sometimes omitted except for those newly defined in this paper. 


\section{$\S 2$ Evolution of cosmological perturbations in the long- wavelength limit}

As mentioned in the introduction, we can determine the dynamical behavior of cosmological perturbations on superhorizon scales by studying an exactly spatially homogeneous system. Since the perturbative analysis of the latter system can be used to determine its Lyapunov exponent, which is an index for a dynamical instability including chaos of a Hamiltonian system, we can also analyse the dynamical instability and integrability of the inflaton dynamics by such a study.

In this section, we summarize the main results of the paper [4] in the case in which the universe is dominated by multi-component scalar fields, and explain how to construct a solution to the perturbation equation in the long-wavelength limit from an exactly homogeneous perturbation of the model. We assume that the universe is spatially flat $(K=0)$ throughout the paper. Hence, the background metric is given by

$$
d s^{2}=-d \tau^{2}+a(\tau)^{2} d \boldsymbol{x}^{2}
$$

We consider the universe dominated by multi-component scalar fields whose energy-momentum tensor is given by

$$
T_{\nu}^{\mu}=\nabla^{\mu} \phi \cdot \nabla_{\nu} \phi-\frac{1}{2} \delta_{\nu}^{\mu}\left(\nabla^{\lambda} \phi \cdot \nabla_{\lambda} \phi+2 U\right)
$$

In the long wavelength limit, the gauge-invariant variable $Y_{i}$ representing the fluctuation of the scalar field $\phi_{i}$ in the flat time slice can be expressed as

$$
\begin{aligned}
Y_{i} & =\chi_{i}+\frac{\dot{\phi}_{i}}{H} \int d \tau \frac{H^{2}}{2 U} W\left(\frac{\dot{\phi}}{H}, \chi\right) ; \\
W\left(X_{1}, X_{2}\right) & :=X_{1} \cdot \dot{X}_{2}-\dot{X}_{1} \cdot X_{2}
\end{aligned}
$$

where $\chi_{i}$ is the combination of the exactly homogeneous perturbation of $\phi_{i}, \delta \phi_{i}$, and the perturbation of the cosmic scale factor $a, \delta a$, given by

$$
\chi_{i}=\delta \phi_{i}-\frac{\dot{\phi}_{i}}{H} \frac{\delta a}{a}
$$

From the equations of motion, it follows that this quantity always satisfies the equation

$$
\frac{a^{3} H^{2}}{U} W\left(\frac{\dot{\phi}}{H}, \chi\right)=\text { const. }
$$

In general, the general solution to the homogenous dynamical system can be expressed in terms of the scale factor $a$ and a set of integration constants as

$$
\phi_{i}=\phi_{i}(a, C) .
$$

For this expression, $\chi_{i}$ can be simpliy written as

$$
\chi_{i}=\frac{\partial \phi_{i}(a, C)}{\partial C} .
$$


As explained in the introduciton, one of the most important quantities describing the evolution behavior of perturbations is the Bardeen parameter defined by

$$
\zeta=\mathcal{R}-\frac{a H}{k} \sigma_{g}
$$

where $\mathcal{R}$ and $\sigma_{g}$ are perturbations of the three curvature and the shear of each constant time slice, respectively. In the present case, the Bardeen parameter $\zeta$ can be written in terms of the gauge-invariant variable $Y_{i}$ as

$$
\zeta=-H \frac{\dot{\phi} \cdot Y}{(\dot{\phi})^{2}} .
$$

Hence, from (2.3),$\zeta$ is represented in the long-wavelength limit as

$$
\zeta=-H \frac{\dot{\phi} \cdot \chi}{(\dot{\phi})^{2}}-\int d \tau \frac{H^{2}}{2 U} W\left(\frac{\dot{\phi}}{H}, \chi\right) .
$$

From (2.5), the second term on the right-hand side of this equation is proportional to $\int d \tau / a^{3}$, which rapidly approaches a constant as the cosmic time $\tau$ increases. Hence, in order to see whether the Bardeen parameter is conserved or not in the superhorizon stage, we can concentrate on the first term. For this reason, from this point, we assume that $\zeta$ is expressed as

$$
\zeta=-H \frac{\dot{\phi} \cdot \chi}{(\dot{\phi})^{2}}
$$

\section{$\S 3$ Evolution equations of corresponding exactly ho- mogeneous universe}

If we use the cosmic scale factor $a$ as the time variable, the action for the homogeneous dynamical system introduced in the previous section can be put into the following Hamiltonian form:

$$
\begin{aligned}
S & =\int \sum_{i} p_{\phi_{i}} d \phi_{i}-h_{a} d a \\
h_{a} & =\frac{2 \sqrt{3}}{\kappa} \Omega a^{2}\left(\frac{1}{2} \frac{1}{\Omega^{2} a^{6}} \sum_{i} p_{\phi_{i}}^{2}+U(\phi)\right)^{1 / 2}, \\
U(\phi) & =\frac{1}{2} \sum_{i} m_{i}^{2} \phi_{i}^{2}+U_{\mathrm{int}}(\phi),
\end{aligned}
$$

where $U_{\text {int }}$ is assumed to be a sum of monomials in $\phi$ of degrees not less than 3 . After changing the time variable to

$$
t=\left(\frac{a}{a_{0}}\right)^{3 / 2}
$$


this action can be expressed in terms of the non-dimensional canonical variables

$$
\Phi=\frac{\phi}{\phi_{0}}, \quad P_{\phi}=\frac{p_{\phi}}{a_{0}^{3} m_{0} \phi_{0} \Omega}
$$

and the dimensionless parameters

$$
\mu_{i}=\frac{m_{i}}{m_{0}}, \quad \epsilon=\frac{\sqrt{3}}{2} \kappa \phi_{0}
$$

as

$$
\begin{aligned}
S^{\prime} & =\frac{S}{\Omega a_{0}^{3} m_{0} \phi_{0}^{2}} \\
& =\int \sum_{i} P_{\phi_{i}} d \Phi_{i}-\frac{2}{\epsilon}\left(\frac{1}{2} \frac{1}{t^{2}} \sum_{i} P_{\phi_{i}}^{2}+\frac{1}{2} t^{2} \sum_{i} \mu_{i}^{2} \Phi_{i}^{2}+t^{2} \frac{1}{m_{0}^{2} \phi_{0}^{2}} U_{\mathrm{int}}\left(\phi_{0} \Phi\right)\right)^{1 / 2} d t(3 .
\end{aligned}
$$

Now, let us introduce the action-angle variables $\left(J_{i}, \theta_{i}\right)$ by

$$
\begin{aligned}
\Phi_{i} & =\sqrt{\frac{2}{\mu_{i}}} \sqrt{J_{i}} \frac{1}{t} \cos \theta_{i} \\
P_{\phi_{i}} & =-\sqrt{2 \mu_{i}} \sqrt{J_{i}} t \sin \theta_{i}
\end{aligned}
$$

which corresponds to the canonical transformation generated by the generating function

$$
W(\Phi, \theta, t)=-\frac{1}{2} \sum_{i} \mu_{i} t^{2} \Phi_{i}^{2} \tan \theta_{i}
$$

Then, the Hamiltonian of the system is transformed to

$$
H=\frac{2}{\epsilon}\left(\sum_{i} \mu_{i} J_{i}+\frac{t^{2}}{m_{0}^{2} \phi_{0}^{2}} U_{\mathrm{int}}\right)^{1 / 2}-\sum_{i} \frac{J_{i}}{t} \sin 2 \theta_{i} .
$$

Further, in terms of these new variable, the Bardeen parameter is expressed as

$$
\begin{aligned}
\zeta= & \frac{2}{3} \frac{\epsilon}{t}\left(\mu \cdot J+\frac{t^{2}}{m_{0}^{2} \phi_{0}^{2}} U_{\mathrm{int}}\right)^{1 / 2} \frac{1}{\sum_{i} \mu_{i} J^{i}\left(1-\cos 2 \theta^{i}\right)} \\
& \sum_{i}\left(\frac{1}{2} \delta J^{i} \sin 2 \theta^{i}-J^{i}\left(1-\cos 2 \theta^{i}\right) \delta \theta^{i}\right)
\end{aligned}
$$

\section{$\S 4 \quad$ Evolution for $t \leq 1 / \epsilon$}

In this section, we evaluate the growth rate of a perturbation of the system defined by the Hamiltonian (3.11) during the period $1 \leq t \leq 1 / \epsilon$, assuming that the masses of scalar fields satisfy a resonant condition approximately. Before going to general arguments, we first explain the basic ideas by two simple models consisting of two scalar fields. 
The first model is defined by

$$
U_{\text {int }}(\phi)=\lambda \phi_{1}^{2} \phi_{2}, \quad 2 \mu_{1} \approx \mu_{2}
$$

If we change the canonical variables from $\left(\theta_{i}, J_{i}\right)(i=0,1)$ to $\left(q_{i}, p_{i}\right)(i=0,1)$ by the linear symplectic transformation

$$
\begin{aligned}
& \theta_{1}=q_{0} \quad \theta_{2}=2 q_{0}+q_{1} \\
& J_{1}=p_{0}-2 p_{1} \quad J_{2}=p_{1},
\end{aligned}
$$

we obtain

$$
\mu_{1} J_{1}+\mu_{2} J_{2}=\omega_{0} p_{0}+\omega_{1} p_{1}
$$

where

$$
\omega_{0}=\mu_{1} \quad \omega_{1}=-2 \mu_{1}+\mu_{2}
$$

$U_{\text {int }}$ can be written

$$
\begin{aligned}
\frac{t^{2}}{m_{0}^{2} \phi_{0}^{2}} U_{\text {int }} & =\frac{\eta}{t} J_{1} J_{2}^{1 / 2}\left\{\cos \left(2 \theta_{1}-\theta_{2}\right)+\cos \left(2 \theta_{1}+\theta_{2}\right)+2 \cos \theta_{2}\right\} \\
& =\frac{\eta}{t}\left(p_{0}-2 p_{1}\right) p_{1}^{1 / 2}\left\{\cos q_{1}+\cos \left(4 q_{0}+q_{1}\right)+2 \cos \left(2 q_{0}+q_{1}\right)\right\},
\end{aligned}
$$

where

$$
\eta=\frac{\lambda \phi_{0}}{m_{0}^{2}} \frac{1}{\mu_{1} \sqrt{\mu_{2}} \sqrt{2}} .
$$

Hence, the contribution of this interaction term to the Hamiltonian is of order $1 / t$, and when averaged over $q_{0}$ and $q_{1}$, it becomes of order $1 / t^{2}$. It generally holds on the interaction terms of the third degree of $\phi$. This feature will play an important role in the argument in $\S 5$.

The second model is defined by

$$
U_{\text {int }}(\phi)=\lambda \phi_{1}^{2} \phi_{2}^{2}, \quad \mu_{1} \approx \mu_{2} .
$$

By the linear symplectic transformation

$$
\begin{aligned}
& \theta_{1}=q_{0} \quad \theta_{2}=q_{0}+q_{1} \\
& J_{1}=p_{0}-p_{1} \quad J_{2}=p_{1},
\end{aligned}
$$

we obtain

$$
\mu_{1} J_{1}+\mu_{2} J_{2}=\omega_{0} p_{0}+\omega_{1} p_{1}
$$

where

$$
\omega_{0}=\mu_{1} \quad \omega_{1}=-\mu_{1}+\mu_{2} .
$$

The interaction term is now written

$$
\begin{aligned}
\frac{t^{2}}{m_{0}^{2} \phi_{0}^{2}} U_{\text {int }}= & \frac{\eta}{t^{2}} J_{1} J_{2}\left\{1+\frac{1}{2} \cos \left(2 \theta_{1}-2 \theta_{2}\right)+\frac{1}{2} \cos \left(2 \theta_{1}+2 \theta_{2}\right)+\cos 2 \theta_{1}+\cos 2 \theta_{2}\right\} \\
= & \frac{\eta}{t^{2}}\left(p_{0}-p_{1}\right) p_{1} \\
& \left\{1+\frac{1}{2} \cos 2 q_{1}+\frac{1}{2} \cos \left(4 q_{0}+2 q_{1}\right)+\cos 2 q_{0}+\cos \left(2 q_{0}+2 q_{1}\right)\right\}
\end{aligned}
$$


where

$$
\eta=\frac{\lambda \phi_{0}^{2}}{m_{0}^{2}} \frac{1}{\mu_{1} \mu_{2}}
$$

Hence, in this model, the contribution of this interaction term to the Hamiltonian is of order $1 / t^{2}$, and its dominant part does not vanish even after it is averaged with respect to $q_{0}$ and $q_{1}$, in contrast to the first model.

In both of these models, $\omega_{1}$ represents the deviation from the exact resonance, which is given by $2 \mu_{1}=\mu_{2}$ for the first model and by $\mu_{1}=\mu_{2}$ for the second model, respectively. Hence, $\omega_{1}$ is much smaller than $\omega_{0}$, and as a consequence, the motion of $q_{0}$ is much faster than that of $q_{1}$ in general. For this reason, we call $q_{0}$ and $q_{1}$ the fast angle variable and the slow angle variable, respectively.

This behavior of the variables $q_{i}$ suggests that the dynamics of the slow variable is well described by a Hamiltonian $\bar{H}$ obtained from $H$ by averaging it with respect to the fast angle variable $q_{0}$ :

$$
\bar{H}=\frac{1}{2 \pi} \int_{0}^{2 \pi} d q_{0} H .
$$

For the first model, this averaged Hamiltonian can be expressed as

$$
\bar{H}=\frac{2}{\epsilon}(\boldsymbol{\omega} \cdot \boldsymbol{p})^{1 / 2}+\frac{\eta}{\epsilon} \frac{1}{t} \frac{1}{(\boldsymbol{\omega} \cdot \boldsymbol{p})^{1 / 2}}\left(p_{0}-2 p_{1}\right) p_{1}^{1 / 2} \cos q_{1}+O\left(\frac{\eta^{2}}{\epsilon} \frac{1}{t^{2}}\right)
$$

and for the second model, as

$$
\bar{H}=\frac{2}{\epsilon}(\boldsymbol{\omega} \cdot \boldsymbol{p})^{1 / 2}+\frac{\eta}{\epsilon} \frac{1}{t^{2}} \frac{1}{(\boldsymbol{\omega} \cdot \boldsymbol{p})^{1 / 2}}\left(p_{0}-p_{1}\right) p_{1}\left\{1+\frac{1}{2} \cos 2 q_{1}\right\}+O\left(\frac{\eta^{2}}{\epsilon} \frac{1}{t^{4}}\right),
$$

where $\boldsymbol{\omega} \cdot \boldsymbol{p}=\omega_{0} p_{0}+\omega_{1} p_{1}$.

This simple procedure, however, does not give a useful approximation, since the higherorder terms with respect to $1 / t$ still contains the large parameter $1 / \epsilon$, as is seen from the above expressions.

Now, we will show for a generic system consisting of $n(\geq 2)$ oscillatory scalar fields that this difficulty can be resolved by taking the average after applying an appropriate canonical transformation to the Hamiltonian. For that purpose, we transform the original action-angle variables to the fast canonical variables $\left(\boldsymbol{q}_{0}, \boldsymbol{p}_{0}\right)$ and the slow variables $\left(\boldsymbol{q}_{1}, \boldsymbol{p}_{1}\right)$, as in the above examples.

For the time being, suppose that the masses of the system satisfy $n_{1}$ resonance relations of the form $\boldsymbol{k} \cdot \boldsymbol{\mu}=0$, where $\boldsymbol{k}$ is a vector with irreducible integer coefficients. Let $R=\left(R_{i j}\right)$ be a unimodular integral matrix such that its last $n_{1}$ rows are given by the $n_{1}$ vectors $\boldsymbol{k}$ defining the resonant relations, and consider the canonical transformation generated by $W=p_{i} R_{i j} \theta_{j}$,

$$
\begin{aligned}
q_{j} & =\frac{\partial W}{\partial p_{j}}=R_{j k} \theta_{k}, \\
J_{j} & =\frac{\partial W}{\partial \theta_{j}}=p_{i} R_{i j} .
\end{aligned}
$$

Here, note that due to the unimodularity of $R$, we can assume that each of the new angle variables $q_{i}$ also has the period $2 \pi$. 
Now, let us decompose these new variables $(\boldsymbol{q}, \boldsymbol{p})$ into two sets of variables as

$$
\begin{aligned}
& \left(\boldsymbol{q}_{0}\right)_{j}=q_{j}, \quad\left(\boldsymbol{p}_{0}\right)_{j}=p_{j} \quad\left(j=1, \cdots, n_{0}\right), \\
& \left(\boldsymbol{q}_{1}\right)_{i}=q_{n_{0}+i}, \quad\left(\boldsymbol{p}_{1}\right)_{i}=p_{n_{0}+i} \quad\left(i=1, \cdots, n_{1}\right),
\end{aligned}
$$

where $n_{0}+n_{1}=n$. Then, the set $\left(\boldsymbol{q}_{0}, \boldsymbol{p}_{0}\right)$ becomes the fast variable and the set $\left(\boldsymbol{q}_{1}, \boldsymbol{p}_{1}\right)$ becomes the slow variable, because $\boldsymbol{\mu} \cdot \boldsymbol{J}$ can be written as

$$
\boldsymbol{\mu} \cdot \boldsymbol{J}=\boldsymbol{p}_{0} \cdot \boldsymbol{\omega}_{0}
$$

where

$$
\left(\boldsymbol{\omega}_{0}\right)_{i}:=R_{i j} \mu_{j} \quad\left(i=1, \cdots, n_{0}\right) .
$$

In the above, we have imposed exact resonant conditions on the mass parameters. However, such strong conditions are rarely satisfied. In fact, it is known that for $d>n-1$, the set of points in the $\boldsymbol{\omega}$ space satisfying the condition

$$
\inf _{\boldsymbol{k} \neq \mathbf{0}, \boldsymbol{k} \in \mathbb{Z}^{n}}|\boldsymbol{k}|^{d}|(\boldsymbol{k} \cdot \boldsymbol{\omega})|=0
$$

has measure zero, where

$$
|\boldsymbol{k}|:=\left|k_{1}\right|+\left|k_{2}\right|+\cdots+\left|k_{n}\right| .
$$

Therefore, from this point, we only require that the mass parameters satisfy resonance conditions approximately. To be precise, we assume that

$$
\begin{aligned}
& \inf _{\boldsymbol{k}_{0} \neq \mathbf{0}, \boldsymbol{k} \in \mathbb{Z}^{n}}|\boldsymbol{k}|^{d}\left|\boldsymbol{k}_{0} \cdot \boldsymbol{\omega}_{0}+\boldsymbol{k}_{1} \cdot \boldsymbol{\omega}_{1}\right|=C>0, \\
& \inf _{\boldsymbol{k} \neq \mathbf{0}, \boldsymbol{k} \in \mathbb{Z}^{n}}|\boldsymbol{k}|^{d}\left|\boldsymbol{k}_{0} \cdot \boldsymbol{\omega}_{0}+\boldsymbol{k}_{1} \cdot \boldsymbol{\omega}_{1}\right| \leq \epsilon C,
\end{aligned}
$$

where $C$ is a constant of order unity. When $\boldsymbol{\omega}$ satisfies (4.24) for some positive constants $d, C$, we say that $\boldsymbol{\omega}$ is of the class $D_{0}(d, C)$.

Next, we show that there exists a canonical transformation such that in a system obtained by that transformation, the dynamics of the slow variables can be determined independent of the fast angle variables with a good accuracy. For that purpose, first note that the Hamiltonian of the system can be written in terms of the variables $\boldsymbol{q}_{0}, \boldsymbol{p}_{0}, \boldsymbol{q}_{1}$ and $\boldsymbol{p}_{1}$ as

$$
H=\frac{2}{\epsilon}\left(\boldsymbol{\omega}_{0} \cdot \boldsymbol{p}_{0}+\boldsymbol{\omega}_{1} \cdot \boldsymbol{p}_{1}\right)^{1 / 2}+A\left(\boldsymbol{q}_{1}, \boldsymbol{p}, t\right)+B(\boldsymbol{q}, \boldsymbol{p}, t),
$$

by Taylor expanding $H$ with respect to $1 / t$, where

$$
\left(\boldsymbol{\omega}_{1}\right)_{i}:=R_{n_{0}+i j} \mu_{j} \quad\left(i=1, \cdots, n_{0}\right) .
$$

We say that this Hamiltonian is of the type $C_{m}\left(\sigma, M_{1}, M_{2}, \rho\right)$, if the following conditions are satisfied for some positive constants $\sigma, M_{1}, M_{2}$, and $\rho$ :

(i) $\boldsymbol{\omega} \cdot \boldsymbol{p}=\boldsymbol{\omega}_{0} \cdot \boldsymbol{p}_{0}+\boldsymbol{\omega}_{1} \cdot \boldsymbol{p}_{1}$ is bounded as

$$
|\boldsymbol{\omega} \cdot \boldsymbol{p}| \geq \sigma
$$


(ii) $t A$ can be extended to an analytic function in the domain $D_{1}(\rho)$ in $\mathbb{C}^{n+n_{1}+1}$ defined by

$$
\begin{aligned}
D(\rho):= & \left\{\left(\boldsymbol{p}, \boldsymbol{q}, \frac{1}{t}\right) ; \operatorname{Re} \boldsymbol{p} \in D+\rho,|\operatorname{Im} \boldsymbol{p}| \leq \rho,|\operatorname{Im} \boldsymbol{q}| \leq \rho,\right. \\
\left.\frac{1}{|t|} \leq 1+\rho,\left|\operatorname{Im} \frac{1}{t}\right| \leq \rho\right\} & \\
D_{1}(\rho):=\left.D(\rho)\right|_{\boldsymbol{q}_{0}=\mathbf{0}} &
\end{aligned}
$$

where $D+\rho$ denotes the $\rho$-neighbourhood of an interval $D$, and in this domain, satisfies the inequality

$$
|t A| \leq M_{1}
$$

Further, $t A$ is periodic with respect to $\boldsymbol{q}_{1}$ and real if $\left(\boldsymbol{q}_{1}, \boldsymbol{p}, 1 / t\right)$ are real.

(iii) $t^{m} B$ can be extended to an analytic function in the domain $D(\rho) \subset \mathbb{C}^{2 n+1}$ defined above and satisfies the inequality

$$
\left|t^{m} B\right| \leq \epsilon^{m-1} M_{2}
$$

Further, $t^{m} B$ is periodic with respect to $\boldsymbol{q}$, real if $(\boldsymbol{q}, \boldsymbol{p}, 1 / t)$ is real, and satisfies

$$
\frac{1}{(2 \pi)^{n_{0}}} \int_{0}^{2 \pi} \cdots \int_{0}^{2 \pi} d^{n_{0}} \boldsymbol{q}_{0} t^{m} B=0 .
$$

Under this notation, the following proposition holds.

Proposition 4.1 Let $m$ be some positive interger, and consider the Hamitonian $H^{(m)}$ written in terms of fast canonical variables $\left(\boldsymbol{q}_{0}^{(m)}, \boldsymbol{p}_{0}^{(m)}\right)$ and slow canonical variables $\left(\boldsymbol{q}_{1}^{(m)}, \boldsymbol{p}_{1}^{(m)}\right)$ as

$$
H^{(m)}=\frac{2}{\epsilon}\left(\boldsymbol{\omega}_{0} \cdot \boldsymbol{p}_{0}^{(m)}+\boldsymbol{\omega}_{1} \cdot \boldsymbol{p}_{1}^{(m)}\right)^{1 / 2}+A_{m}\left(\boldsymbol{q}_{1}^{(m)}, \boldsymbol{p}^{(m)}, t\right)+B_{m}\left(\boldsymbol{q}^{(m)}, \boldsymbol{p}^{(m)}, t\right) .
$$

Suppose that $\boldsymbol{\omega}$ is of the class $D_{0}(d, C)$ and that this Hamiltonian is of the type $C_{m}\left(\sigma_{m}, M_{1}^{(m)}, M_{2}^{(m)}, \rho_{m}\right)$. Then, for any $\delta>0$, there exists $\epsilon_{0}>0$ such that, for an arbitrary $\epsilon$ satisfying

$$
0<\epsilon<\epsilon_{0},
$$

there exists a function $S_{m}(\boldsymbol{q}, \boldsymbol{p}, t)$ satisfying the following conditions:

(i) $S_{m}$ is periodic with respect to $\boldsymbol{q}$ and real if $(\boldsymbol{q}, \boldsymbol{p}, t)$ is real.

(ii) $t^{m} S_{m}$ can be extended to an analytic function in the multi-dimensional complex domain $D\left(\rho_{m+1}\right)$, where $\rho_{m+1}=\rho_{m}-\delta$, and in this domain, satisfies the inequality

$$
\left|t^{m} S_{m}\right| \leq \epsilon^{m} L_{1}^{(m)}
$$

for some positive constant $L_{1}^{(m)}$. 
(iii) Let $H^{(m+1)}\left(\boldsymbol{q}^{(m+1)}, \boldsymbol{p}^{(m+1)}, t\right)$ be a Hamitonian obtained from $H^{(m)}$ by the canonical transformation generated by $S_{m}\left(\boldsymbol{q}^{(m)}, \boldsymbol{p}^{(m+1)}, t\right)$ :

$$
\begin{aligned}
& \boldsymbol{p}^{(m)}=\boldsymbol{p}^{(m+1)}+\frac{\partial S_{m}}{\partial \boldsymbol{q}^{(m)}} \\
& \boldsymbol{q}^{(m+1)}=\boldsymbol{q}^{(m)}+\frac{\partial S_{m}}{\partial \boldsymbol{p}^{(m+1)}} \\
& H^{(m+1)}=H^{(m)}+\frac{\partial S_{m}}{\partial t} \\
& \quad=\frac{2}{\epsilon}\left(\boldsymbol{\omega} \cdot \boldsymbol{p}^{(m+1)}\right)^{1 / 2}+A_{m+1}\left(\boldsymbol{q}_{1}^{(m+1)}, \boldsymbol{p}^{(m+1)}, t\right)+B_{m+1}\left(\boldsymbol{q}^{(m+1)}, \boldsymbol{p}^{(m+1)}, t\right)
\end{aligned}
$$

Then, $H^{(m+1)}$ is of the type $C_{m+1}\left(\sigma_{m+1}, M_{1}^{(m+1)}, M_{2}^{(m+1)}, \rho_{m+1}\right)$ for some positive constants $\sigma_{m+1}, M_{1}^{(m+1)}$, and $M_{2}^{(m+1)}$, and the change of the $A$-term in the Hamiltonians satisfies the inequality

$$
\left|t^{m+1}\left\{A_{m+1}\left(\boldsymbol{q}_{1}, \boldsymbol{p}, t\right)-A_{m}\left(\boldsymbol{q}_{1}, \boldsymbol{p}, t\right)\right\}\right| \leq \frac{\epsilon^{m}}{2} M_{2}^{(m+1)},
$$

for $\left(\boldsymbol{q}_{1}, \boldsymbol{p}, 1 / t\right) \in D_{1}\left(\rho_{m+1}\right)$.

(For the proof, see the appendix $A$ ).

Since the generating function $S_{m}$ in this proposition is of the order $\epsilon^{m} / t^{m}$, we have $B_{m} \sim$ $\epsilon^{m-1} / t^{m}$ and $B_{m+1} \sim \epsilon^{m} / t^{m+1}$. This implies that we can make the part of the Hamiltonian that depends on the fast variables $\boldsymbol{q}_{0}$ aribitrarily small by taking the original Hamiltonian as the starting point $(m=1)$ and applying canonical tranformations given in the proposition repeatedly. Therefore, we can expect that the evolution of the slow variables $\left(\boldsymbol{q}_{1}, \boldsymbol{p}_{1}\right)$ can be determined with a quite good accuracy from an effective Hamiltonian $\bar{H}^{(m)}\left(\boldsymbol{q}_{1}^{(m)}, \boldsymbol{p}^{(m)}, t\right)$ obtained by dicarding $B_{m}$ term from $H^{(m)}$, if we take $m$ sufficiently large. In this trancated system, $\boldsymbol{p}_{0}^{(m)}$ becomes constant, and the behavior of $\boldsymbol{q}_{0}^{(m)}$ can be obtained by a simple time integration of a function that has a definite $t$-dependence when $\boldsymbol{p}_{0}^{(m)}$ and a corresponding solution for $\left(\boldsymbol{q}_{1}^{(m)}, \boldsymbol{p}_{1}^{(m)}\right)$ are given. The evolution of the original variables can be calculated from this solution by applying the known canonical transformation connecting these two sets of variables.

Next, we show that this expectation is correct by estimating the errors produced by the trunction. Let us use the symbol $\Delta$ to represent the difference of a quantity for the exact system and the corresponding quantity for the truncated system. Further, let us denote a quantity for the truncated system by the upper case letter of the lower case symbol representing the corresponding quantity for the exact system. For example, the errors of canonical variables are denoted as

$$
\begin{aligned}
\Delta Q & =q-Q \\
\Delta P & =p-P
\end{aligned}
$$

and the errors of solutions to the perturbation equations for both systems are denoted as

$$
\begin{aligned}
\Delta \delta \boldsymbol{Q} & =\delta \boldsymbol{q}-\delta \boldsymbol{Q}, \\
\Delta \delta \boldsymbol{P} & =\delta \boldsymbol{p}-\delta \boldsymbol{P} .
\end{aligned}
$$


Further, let us denote the sets of slow variables, $\left(\boldsymbol{q}_{1}, \boldsymbol{p}_{1}\right)$ and $\left(\boldsymbol{Q}_{1}, \boldsymbol{P}_{1}\right)$, by $\boldsymbol{z}$ and $\boldsymbol{Z}$, respectively. Finally, for a function $f(t)$, let us define $\|f\|(t)$ by

$$
\|f\|(t)=\sup _{1 \leq s \leq t}|f(s)| .
$$

Before evaluating $\Delta \boldsymbol{Z}, \Delta \delta \boldsymbol{Z}$ and $\delta \boldsymbol{Z}$, we give the general technique to evaluate an upper bound on the norm of the solution $\boldsymbol{X}$ to the first order differential equation:

$$
\frac{d}{d t} \boldsymbol{X}=\Omega \boldsymbol{X}+\boldsymbol{S}
$$

where $\boldsymbol{X}, \boldsymbol{S}$ are $N$ column vectors and $\Omega$ is $N \times N$ matrix. For the detail, see the appendix of the paper [7]. If we define the norm of the solution $\boldsymbol{X}$ by

$$
|\boldsymbol{X}|^{2}:=\boldsymbol{X}^{\dagger} \boldsymbol{X}
$$

we decompose $\Omega$ into a sum of $\Omega_{1}, \Omega_{2}$ : $\Omega_{1}$ from the perturbed part is of order $1 / t$ and $\Omega_{2}$ from the unperturbed part is of order $\epsilon$ and $\lambda_{m i}$ is the largest eigenvalue of the hermitian matrix $\Omega_{H i}:=\Omega_{i}^{\dagger}+\Omega_{i},|\boldsymbol{X}|$ is bounded as

$$
\frac{d}{d t}|\boldsymbol{X}| \leq \frac{\lambda}{2}|\boldsymbol{X}|+|\boldsymbol{S}|
$$

where

$$
\lambda:=\lambda_{m 1}+\lambda_{m 2} .
$$

If $\lambda$ is an eigenvalue of $\Omega_{H i},-\lambda$ is also an eigenvalue of $\Omega_{H i}$, because $\Omega_{i}$ can be written in the form of $I S_{i}$ where $S_{i}$ is the symmetric matrix and $I$ is defined by (4.79). So $\lambda_{m i}$ are non-negative. Therefore the norm of the solution is bounded as

$$
|\boldsymbol{X}| \leq \exp \left[\int_{1} \frac{\lambda}{2} d t\right]\left[|\boldsymbol{X}(1)|+\int_{1} d t|\boldsymbol{S}|\right] .
$$

In evaluation of the contribution from the source term, we use

$$
\left|\sum_{i=1}^{k} \boldsymbol{S}_{i}\right| \leq \sum_{i=1}^{k}\left|\boldsymbol{S}_{i}\right|
$$

which is obtained from the Cauchy Schwarz inequality

$$
\left|\boldsymbol{A}^{\dagger} \boldsymbol{B}\right| \leq|\boldsymbol{A}||\boldsymbol{B}|
$$

Since the different definition of the norm

$$
|\boldsymbol{X}|_{m}:=\max _{1 \leq i \leq N}\left|(\boldsymbol{X})_{i}\right|
$$

satisfies

$$
\frac{1}{\sqrt{N}}|\boldsymbol{X}| \leq|\boldsymbol{X}|_{m} \leq|\boldsymbol{X}|
$$

we can identify $|\boldsymbol{X}|$ and $|\boldsymbol{X}|_{m}$ assuming that the number of degrees of freedom $N$ is of order unity, so we simply omit the subscripts $m$.

Then, the truncation error for the fourth-order system can be estimated as follows. From now on we omit constant coefficients of order unity except $\Gamma$. 
Proposition 4.2A Let $\Gamma$ be an upper bound of the eigenvalues of the the hermitian matrix $t \Omega_{H 1} / 2$ defined by

$$
\begin{aligned}
\Omega_{H 1} & :=\Omega_{1}^{\dagger}+\Omega_{1}, \\
\Omega_{1} & :=\left(\begin{array}{cc}
\frac{\partial^{2} A}{\partial \boldsymbol{Q}_{1} \partial \boldsymbol{P}_{1}} & \frac{\partial^{2} A}{\partial \boldsymbol{P}_{1} \partial \boldsymbol{P}_{1}} \\
-\frac{\partial^{2} A}{\partial \boldsymbol{Q}_{1} \partial \boldsymbol{Q}_{1}} & -\frac{\partial^{2} A}{\partial \boldsymbol{P}_{1} \partial \boldsymbol{Q}_{1}}
\end{array}\right),
\end{aligned}
$$

where $A$ is the $A$-term of the fourth-order Hamiltonian $H=H^{(4)}$, and all the elements of $\Omega_{1}$ are bounded by $M_{1}^{(4)} / \delta^{2} t$ in the domain $D_{1}\left(\rho_{4}-\delta\right)$. This quantity $\Gamma$ gives an upper bound on the growth rates of the errors $\Delta \boldsymbol{Q}_{1}, \Delta \boldsymbol{P}_{1}, \Delta \delta \boldsymbol{Q}_{1}$, and $\Delta \delta \boldsymbol{P}_{1}$, and the growth of the perturbations $\delta \boldsymbol{Q}_{1}, \delta \boldsymbol{P}_{1}$.

Let us define $\beta$ by

$$
\beta=\frac{1}{\Gamma+1},
$$

Then, for an arbitrary positive $\epsilon$, in the time interval

$$
1 \leq t \leq \frac{1}{\epsilon^{\beta}}
$$

the truncation errors of canonical variables are given by

$$
\left|\Delta \boldsymbol{P}_{0}\right| \leq \epsilon^{3}, \quad|\Delta \boldsymbol{Z}| \leq \epsilon^{2}, \quad\left|\frac{\Delta \boldsymbol{Q}_{0}}{t}\right| \leq \epsilon^{2},
$$

and the errors of perturbation variables are

$$
\begin{aligned}
\left\|\frac{\Delta \delta \boldsymbol{Q}_{0}}{t}\right\| & \leq \epsilon^{2}\left|\delta \boldsymbol{Q}_{0}(1)\right|+\epsilon\left\|\delta \boldsymbol{P}_{0}\right\|+\epsilon\|\delta \boldsymbol{Z}\|, \\
\left\|\Delta \delta \boldsymbol{P}_{0}\right\| & \leq \epsilon^{3}\left|\delta \boldsymbol{Q}_{0}(1)\right|+\epsilon^{2}\left\|\delta \boldsymbol{P}_{0}\right\|+\epsilon^{3}\|\delta \boldsymbol{Z}\|, \\
\|\Delta \delta \boldsymbol{Z}\| & \leq \epsilon^{2}\left|\delta \boldsymbol{Q}_{0}(1)\right|+\epsilon\left\|\delta \boldsymbol{P}_{0}\right\|+\epsilon\|\delta \boldsymbol{Z}\|,
\end{aligned}
$$

under the initial conditions:

$$
\begin{aligned}
& \Delta \boldsymbol{P}_{0}(1)=\Delta \boldsymbol{Z}(1)=\Delta \boldsymbol{Q}_{0}(1)=0, \\
& \Delta \delta \boldsymbol{Q}_{0}(1)=\Delta \delta \boldsymbol{P}_{0}(1)=\Delta \delta \boldsymbol{Z}(1)=0 .
\end{aligned}
$$

(For the proof see Appendix B.1.)

This proposition shows that the truncation errors can be made small in the fourth-order system $(m=4)$. In order to obtain the information on the behavior of the original variables, we also have to estimate how this truncation error affects the original variables through the canonical transformation which connects the fourth-order system and the original system. The next proposition gives that estimate. 
Proposition $4.2 B$ The difference between $\left(\boldsymbol{q}^{(1)}, \boldsymbol{p}^{(1)}\right)$ obtained from $\left(\boldsymbol{q}^{(4)}, \boldsymbol{p}^{(4)}\right)$ by the canonical transformation and $\left(\boldsymbol{Q}^{(1)}, \boldsymbol{P}^{(1)}\right)$ obtained from $\left(\boldsymbol{Q}^{(4)}, \boldsymbol{P}^{(4)}\right)$ by the same canonical transformation has the upper bound

$$
\left|\Delta \boldsymbol{P}_{0}^{(1)}\right| \leq \epsilon^{3}, \quad\left|\Delta \boldsymbol{Z}^{(1)}\right| \leq \epsilon^{2}, \quad\left|\frac{\Delta \boldsymbol{Q}_{0}^{(1)}}{t}\right| \leq \epsilon^{2},
$$

and the corresponding difference in the perturbation variables is estimated as

$$
\begin{aligned}
\left\|\frac{\Delta \delta \boldsymbol{Q}_{0}^{(1)}}{t}\right\| & \leq \epsilon^{2}\left|\delta \boldsymbol{Q}_{0}^{(4)}(1)\right|+\epsilon\left\|\delta \boldsymbol{P}_{0}^{(4)}\right\|+\epsilon\left\|\delta \boldsymbol{Z}^{(4)}\right\|, \\
\left\|\Delta \delta \boldsymbol{P}_{0}^{(1)}\right\| & \leq \epsilon^{2}\left|\delta \boldsymbol{Q}_{0}^{(4)}(1)\right|+\epsilon\left\|\delta \boldsymbol{P}_{0}^{(4)}\right\|+\epsilon^{2}\left\|\delta \boldsymbol{Z}^{(4)}\right\|, \\
\left\|\Delta \delta \boldsymbol{Z}^{(1)}\right\| & \leq \epsilon^{2}\left|\delta \boldsymbol{Q}_{0}^{(4)}(1)\right|+\epsilon\left\|\delta \boldsymbol{P}_{0}^{(4)}\right\|+\epsilon\left\|\delta \boldsymbol{Z}^{(4)}\right\|,
\end{aligned}
$$

in the same time interval as in the previous proposition. (For the proof see Appendix B.2.)

These two propositions show that the truncation errors can be made small even with respect to the original variables, if we truncate the system at the fourth-order. This order is minimal in the sense that the trunction at a lower-order system produces errors of order unity in the perturbation variables. Conversely, if we go to higher-order systems, we can obtain a better approximation. Further, we can prolong the time interval in which the approximation is good. In fact, we can show that in the $m$-th order system with $m \geq 4$, the same estimates for the truncation errors as in the above propositions hold in the interval

$$
1 \leq t \leq \frac{1}{\epsilon^{(m-3) /(\Gamma+1)}} .
$$

In particular, for $m$ larger than $\Gamma+4$, the approximation is good in the interval $1<t<1 / \epsilon$.

Finally, let us evaluate the growth of perturbations of a truncated higher-order system. The Hamiltonian of such a system can be in general expressed as

$$
H=\frac{2}{\epsilon}\left(\boldsymbol{\omega}_{0} \cdot \boldsymbol{P}_{0}+\boldsymbol{\omega}_{1} \cdot \boldsymbol{P}_{1}\right)^{1 / 2}+A\left(\boldsymbol{Q}_{1}, \boldsymbol{P}, t\right),
$$

where

$$
|t A| \leq M, \quad \boldsymbol{\omega}_{1}=O(\epsilon) .
$$

As mentioned before, the fast action variables $\boldsymbol{P}_{0}$ for this system become constants of motion, and the equations of motion for the slow variables $\left(\boldsymbol{P}_{1}, \boldsymbol{Q}_{1}\right)$ do not contain small parameter $\epsilon$ essentially. Therefore, in contrast to $\boldsymbol{P}_{0}$, the slow action variables $\boldsymbol{P}_{1}$ are not conserved in general, and as a consequence, the Bardeen parameter can grow considerably in the time interval $1 \leq t \leq 1 / \epsilon$. (For estimate of the growth rates of the Bardeen parameter in the concrete examples, see the Appendix E.) This can be confirmed by the following estimate of the upper bound of the perturbation variables $\delta \boldsymbol{Q}$ and $\delta \boldsymbol{P}$. 
Proposition 4.3 In the time interval $1 \leq t \leq 1 / \epsilon$, the perturbation variables $\delta \boldsymbol{Q}$ and $\delta \boldsymbol{P}$ are bounded as

$$
\begin{aligned}
|\delta \boldsymbol{Z}| \leq & t^{\Gamma}\left[|\delta \boldsymbol{Z}(1)|+\left|\delta \boldsymbol{P}_{0}(1)\right|(t-1)\right] \\
\left|\delta \boldsymbol{P}_{0}\right|= & \left|\delta \boldsymbol{P}_{0}(1)\right| \\
\left|\delta \boldsymbol{Q}_{0}\right| \leq & \left|\delta \boldsymbol{Q}_{0}(1)\right|+\frac{1}{\epsilon}\left|\delta \boldsymbol{P}_{0}(1)\right|(t-1) \\
& +|\delta \boldsymbol{Z}(1)| \frac{t^{\Gamma+1}-1}{\Gamma+1}+\left|\delta \boldsymbol{P}_{0}(1)\right|\left(\frac{t^{\Gamma+2}-1}{\Gamma+2}-\frac{t^{\Gamma+1}-1}{\Gamma+1}\right),
\end{aligned}
$$

where $\Gamma$ is the quantity defined in the proposition $4.2 \mathrm{~B}$. In particular, the growth rate of the perturbation variables is not exponential and at most a power of $t$.

proof The evolution of the slow variables $\delta \boldsymbol{Q}_{1}$ and $\delta \boldsymbol{P}_{1}$ is determined by the equation

$$
\begin{aligned}
\frac{d}{d t}\left(\begin{array}{c}
\delta \boldsymbol{Q}_{1} \\
\delta \boldsymbol{P}_{1}
\end{array}\right)= & \left(\begin{array}{cc}
\frac{\partial^{2} A}{\partial \boldsymbol{Q}_{1} \partial \boldsymbol{P}_{1}} & -\frac{\boldsymbol{\omega}_{1} \cdot \boldsymbol{\omega}_{1}}{2 \epsilon(\boldsymbol{\omega} \cdot \boldsymbol{P})^{3 / 2}}+\frac{\partial^{2} A}{\partial \boldsymbol{P}_{1} \partial \boldsymbol{P}_{1}} \\
-\frac{\partial^{2} A}{\partial \boldsymbol{Q}_{1} \partial \boldsymbol{Q}_{1}} & -\frac{\partial^{2} A}{\partial \boldsymbol{P}_{1} \partial \boldsymbol{Q}_{1}}
\end{array}\right)\left(\begin{array}{c}
\delta \boldsymbol{Q}_{1} \\
\delta \boldsymbol{P}_{1}
\end{array}\right) \\
& +\delta \boldsymbol{P}_{0}\left(\begin{array}{c}
-\frac{\boldsymbol{\omega}_{1} \cdot \boldsymbol{\omega}_{0}}{2 \epsilon(\boldsymbol{\omega} \cdot \boldsymbol{P})^{3 / 2}}+\frac{\partial^{2} A}{\partial \boldsymbol{P}_{0} \partial \boldsymbol{P}_{1}} \\
-\frac{\partial^{2} A}{\partial \boldsymbol{P}_{0} \partial \boldsymbol{Q}_{1}}
\end{array}\right) .
\end{aligned}
$$

By evaluating the coefficients in the right-hand side, we obtain

$$
\frac{d}{d t}|\delta \boldsymbol{Z}| \leq\left(\frac{\Gamma}{t}+\epsilon\right)|\delta \boldsymbol{Z}|+\left|\delta \boldsymbol{P}_{0}(1)\right|
$$

where we have used $\delta \boldsymbol{P}_{0}=\delta \boldsymbol{P}_{0}(1)$. By integrating this inequality, we obtain

$$
|\delta \boldsymbol{Z}| \leq t^{\Gamma} \exp [\epsilon(t-1)]\left[|\delta \boldsymbol{Z}(1)|+\left|\delta \boldsymbol{P}_{0}(1)\right|(t-1)\right] .
$$

Since we are considering the time interval $1 \leq t \leq 1 / \epsilon$, we have

$$
|\delta \boldsymbol{Z}| \leq t^{\Gamma}\left[|\delta \boldsymbol{Z}(1)|+\left|\delta \boldsymbol{P}_{0}(1)\right|(t-1)\right]
$$

Next, $\delta \boldsymbol{Q}_{0}$ obeys the equation

$$
\begin{aligned}
\frac{d}{d t} \delta \boldsymbol{Q}_{0}= & -\frac{1}{2} \frac{\boldsymbol{\omega}_{0}}{\epsilon} \frac{1}{(\boldsymbol{\omega} \cdot \boldsymbol{P})^{3 / 2}}\left(\boldsymbol{\omega}_{0} \cdot \delta \boldsymbol{P}_{0}+\boldsymbol{\omega}_{1} \cdot \delta \boldsymbol{P}_{1}\right) \\
& +\left(\delta \boldsymbol{P}_{0} \cdot \frac{\partial}{\partial \boldsymbol{P}_{0}}+\delta \boldsymbol{Q}_{1} \cdot \frac{\partial}{\partial \boldsymbol{Q}_{1}}+\delta \boldsymbol{P}_{1} \cdot \frac{\partial}{\partial \boldsymbol{P}_{1}}\right) \frac{\partial A}{\partial \boldsymbol{P}_{0}}
\end{aligned}
$$

Integrating the right-hand side of this equation with respect to $t$ yields

$$
\begin{aligned}
\left|\delta \boldsymbol{Q}_{0}\right| & \leq\left|\delta \boldsymbol{Q}_{0}(1)\right|+\frac{1}{\epsilon} \int_{1} d t\left|\delta \boldsymbol{P}_{0}(1)\right|+\int_{1} d t\left|\delta \boldsymbol{P}_{1}\right|+\int_{1} d t \frac{1}{t}\left[\left|\delta \boldsymbol{P}_{0}(1)\right|+|\delta \boldsymbol{Z}|\right] \\
& \leq\left|\delta \boldsymbol{Q}_{0}(1)\right|+\frac{1}{\epsilon} \int_{1} d t\left|\delta \boldsymbol{P}_{0}(1)\right|+\int_{1} d t|\delta \boldsymbol{Z}|
\end{aligned}
$$


From (4.74) and this equation, we obtain (4.70).

Next, we show that the above general estimate of the upper bound on the growth of perturbations is rather good, by presenting an example in which the upper bound is nearly saturated. Let us consider a system in which the Hamiltonian flow in the phase space $\left(\boldsymbol{Q}_{1}, \boldsymbol{P}_{1}\right)$ has a equilibrium fixed point. For simplicity, we assume the exact resonance condition $\boldsymbol{\omega}_{1}=\mathbf{0}$. Then, in terms of the phase space variable $\boldsymbol{Z}$ defined by

$$
\boldsymbol{Z}^{i}=\boldsymbol{Q}_{1}^{i} \quad\left(1 \leq i \leq n_{1}\right), \quad \boldsymbol{Z}^{n_{1}+i}=\boldsymbol{P}_{1}^{i} \quad\left(1 \leq i \leq n_{1}\right)
$$

the Hamiltonian equations of motion can be written

$$
\frac{d \boldsymbol{Z}}{d t}=I \frac{\partial H}{\partial \boldsymbol{Z}}
$$

where $I$ is the matrix of degree $2 n_{1}$ expressed in terms of the unit matrix $E$ of degree $n_{1}$ as

$$
I=\left(\begin{array}{cc}
0 & E \\
-E & 0
\end{array}\right) .
$$

We consider the Hamiltonian of the form

$$
H=\frac{2}{\epsilon}\left(\boldsymbol{\omega}_{0} \cdot \boldsymbol{P}_{0}\right)^{1 / 2}+\frac{1}{t^{\gamma}} a\left(\boldsymbol{Q}_{1}, \boldsymbol{P}\right),
$$

where $\gamma=1$ or $\gamma=2$. When the resonant interactions come from interaction terms of the third order in the scalar fields $\phi, \gamma=1$, and when they come from interaction terms of the fourth order in $\phi, \gamma=2$. We focus on the dynamical behavior near the fixed point $\left(\boldsymbol{P}_{0}(1), \boldsymbol{Z}(1)\right)$ and expand $a\left(\boldsymbol{P}_{0}, \boldsymbol{Z}\right)$ as

$$
a=a_{0}+\boldsymbol{b}^{T} \underline{\boldsymbol{P}_{0}}+\frac{1}{2}{\underline{\boldsymbol{P}_{0}}}^{T} C \underline{\boldsymbol{P}_{0}}+\underline{\boldsymbol{Z}}^{T} D \underline{\boldsymbol{P}_{0}}+\frac{1}{2} \underline{\boldsymbol{Z}}^{T} F \underline{\boldsymbol{Z}},
$$

dropping the terms of degree not less than 3 with respect to deviations from the equilibrium; $\underline{\boldsymbol{P}_{0}}=\boldsymbol{P}_{0}-\boldsymbol{P}_{0}(1), \underline{\boldsymbol{Z}}=\boldsymbol{Z}-\boldsymbol{Z}(1)$, where $T$ implies to take the transposition of a matrix, $\overline{a_{0}}$ is a constant, $\boldsymbol{b}$ and $\boldsymbol{P}_{0}$ are $n_{0}$-dimensional vectors, $C$ is an $n_{0} \times n_{0}$ symmetric matrix, $D$ is a $2 n_{1} \times n_{0}$ matrix, and $F$ is a $2 n_{1} \times 2 n_{1}$ symmetric matrix. Since $\boldsymbol{Z}(1)$ is the fixed points, $a$ does not contain $\boldsymbol{g}^{T} \underline{\boldsymbol{Z}}$. Then, the evolution equations of the slow variables $\underline{\boldsymbol{Z}}$ can be written in the matrix form as

$$
\frac{d \underline{\boldsymbol{Z}}}{d t}=\frac{1}{t^{\gamma}}\left[I F \underline{\boldsymbol{Z}}+I D \underline{\boldsymbol{P}_{0}}\right]
$$

Taking the variation of this equation and using the relations

$$
\delta \boldsymbol{P}_{0}=\delta \boldsymbol{P}_{0}(1)
$$

we obtain

$$
\frac{d}{d t} \delta \boldsymbol{Z}=\frac{1}{t^{\gamma}}\left[I F \delta \boldsymbol{Z}+I D \delta \boldsymbol{P}_{0}(1)\right]
$$

Here, note that the $2 n_{1} \times 2 n_{1}$ matrix $X:=I F$ satisfies the equation

$$
X^{T}=I X I .
$$


From this and $I^{2}=-1$, it follows that the characteristic polynomial

$$
\Delta(\lambda)=\operatorname{det}(X-\lambda E)
$$

is an even function of $\lambda$ :

$$
\Delta(\lambda)=\Delta(-\lambda) .
$$

In addition, since $\Delta(\lambda)$ is real polynomial, we have

$$
\Delta(\lambda)=\Delta\left(\lambda^{*}\right) .
$$

Hence, if $a+b i,(a, b$ real $)$ is an eigenvalue of $X$, all of $\pm a \pm b i$ are also eigenvalues of $X$. From this, it follows that if $X$ has an eigenvalue with a non-vanishing real part, the fixed point is unstable. We say that such a fixed point is hyperbolic. In constrast, if all eigenvalues of $X$ are pure imaginary, the fixed point is stable. We say that such a fixed point is elliptic. In the case in which the fixed point is neither hyperbolic nor elliptic, i.e., some of the eigenvalues of $X$ vanish and the other eigenvalues are pure imaginary, the flow around the fixed point is stable in the linear analysis but may become unstable if higher-order terms are taken into account.

To be precise, this stability argument applies to this autonomous systems. In the present case, due to the existence of the time-dependent factor $1 / t^{\gamma}$, the real stability depends on the value of $\gamma$. Since the system under consideration is linear, we can check it directly by solving the equation. First, by diagonalizing the matrix $X$ as

$$
S^{-1} X S=\Lambda,
$$

where $\Lambda$ is a diagonal matrix, the general solution for $\delta Z$ can be written

$$
\delta \boldsymbol{Z}=S \exp \left[\int_{1} \frac{d t}{t^{\gamma}} \Lambda\right] S^{-1} \boldsymbol{K}-X^{-1} I D \delta \boldsymbol{P}_{0}(1),
$$

where

$$
\boldsymbol{K}=\delta \boldsymbol{Z}(1)+X^{-1} I D \delta \boldsymbol{P}_{0}(1) .
$$

Next, the equation for $\delta \boldsymbol{Q}_{0}$ is given by

$$
\frac{d}{d t} \delta \boldsymbol{Q}_{0}=-\frac{1}{2} \frac{\boldsymbol{\omega}_{0}}{\epsilon} \frac{\boldsymbol{\omega}_{0} \cdot \delta \boldsymbol{P}_{0}(1)}{\left(\boldsymbol{\omega}_{0} \cdot \boldsymbol{P}_{0}(1)\right)^{1 / 2}}+\frac{1}{t^{\gamma}}\left(C \delta \boldsymbol{P}_{0}(1)+\delta \boldsymbol{Z}^{T} D\right) .
$$

Hence, $\delta \boldsymbol{Q}_{0}$ can be expressed as

$$
\delta \boldsymbol{Q}_{0}=\delta \boldsymbol{Q}_{0}(1)-\frac{\boldsymbol{\omega}_{0}}{2 \epsilon} \frac{\boldsymbol{\omega}_{0} \cdot \delta \boldsymbol{P}_{0}(1)}{\left(\boldsymbol{\omega}_{0} \cdot \boldsymbol{P}_{0}(1)\right)^{1 / 2}}(t-1)+\boldsymbol{R},
$$

where

$$
\boldsymbol{R}=C \delta \boldsymbol{P}_{0}(1) \int_{1} \frac{d t}{t^{\gamma}}+\int_{1} \frac{d t}{t^{\gamma}} \delta \boldsymbol{Z}^{T} D
$$

When $\gamma=1$, inserting the above expression for $\delta \boldsymbol{Z}$ into this equation, we obtain

$$
|\boldsymbol{R}| \leq \frac{t^{\lambda}-1}{\lambda}+\ln t
$$


for $\lambda>0$, and

$$
|\boldsymbol{R}| \leq \ln t
$$

for $\lambda=0$, where $\lambda$ is the maximum of the real parts of the eigenvalues of $X$. Thus, $\delta \boldsymbol{Q}_{0}$ is always unstable. In contrast, when $\gamma=2,|\boldsymbol{R}|$ is bounded from above as

$$
|\boldsymbol{R}| \leq e^{\lambda}+1
$$

and the stability of $\delta \boldsymbol{Q}_{0}$ depends on the value of $\delta \boldsymbol{P}_{0}$.

Finally, we give an exactly soluble example such that there appear both hyperbolic fixed points and elliptic fixed points and the phase flow pattern is similar to that of the pendulum in a conservative field. It is given by the Hamiltonian

$$
H=\frac{2}{\epsilon}\left(\omega_{0} P_{0}\right)^{1 / 2}+\frac{1}{t^{2}}\left(\frac{1}{2} a P_{1}^{2}+b \cos Q_{1}\right)+\frac{c}{t^{2}},
$$

where $a, b, c$ are positive functions of $P_{0}$.

Since there exists a conserved quantity,

$$
C=\frac{1}{2} a P_{1}^{2}+b \cos Q_{1}=\text { const, }
$$

in this model, we can determine the phase flow pattern in the $\left(Q_{1}, P_{1}\right)$ plane easily. In particular, we find that the phase plain is divided into a region of oscillatory motions and a region of rotationary motions by the separatrix defined by

$$
P_{1}= \pm 2 \sqrt{\frac{b}{a}} \sin \frac{Q_{1}}{2}
$$

Further, we find two types of fixed points. One is elliptic points, $P_{1}=0, Q_{1}=(2 k+1) \pi$, where $k$ is an integer, each of which is surrounded by flows corresponding to oscillatory motions. The other is hyperbolic fixed points, $P_{1}=0, Q_{1}=2 \pi k$, where $k$ is an integer. These fixed points are connected by the heteroclinic orbits (4.100). This implies that near the heteroclinic orbits, the perturbations $\delta Q$ and $\delta P$, therefore the Bardeen parameter grows.

\section{$\S 5 \quad$ Evolution for $t \geq 1 / \epsilon$}

We have treated the case in which $\boldsymbol{\omega}$ is of the class $D_{0}(d, C)$ and satisfies the resonance relations at least approximately (4.25) for $t \leq 1 / \epsilon$. When there exists deviation from the exact resonance, as the time proceeds, the instability characteristic of resonance disappears and the evolution of the system is reduced to the superposition of oscillations with amplitudes and frequencies of different orders. We demonstrate this fact by investigating the case in which $\boldsymbol{\omega}$ is of the classes $D_{0}(d, C)$ and $D(d, \epsilon C)$ for $t \geq 1 / \epsilon$ : we say that $\boldsymbol{\omega}$ is of the class $D(d, C)$ if

$$
\inf _{\boldsymbol{k} \neq \mathbf{0}, \boldsymbol{k} \in \mathbb{Z}^{n}}|\boldsymbol{k}|^{d}\left|\boldsymbol{k}_{0} \cdot \boldsymbol{\omega}_{0}+\boldsymbol{k}_{1} \cdot \boldsymbol{\omega}_{1}\right|=C,
$$

is satisfied for some positive constants $d, C$. 
By redefining $t, H$ by $\epsilon t, H / \epsilon$ respectively, we investigate the evolutionary behavior for $t \geq 1$ of the system defined by the Hamiltonian of the type $C_{1,1}\left(\sigma, M_{11}, M_{12}, M_{2}, \rho\right)$ : we say that this Hamiltonian

$$
\begin{aligned}
H & =\frac{2}{\epsilon^{2}}(\boldsymbol{\omega} \cdot \boldsymbol{p})^{1 / 2}+A\left(\boldsymbol{q}_{1}, \boldsymbol{p}, t\right)+B(\boldsymbol{q}, \boldsymbol{p}, t), \\
A\left(\boldsymbol{q}_{1}, \boldsymbol{p}, t\right) & =D(\boldsymbol{p}, t)+E\left(\boldsymbol{q}_{1}, \boldsymbol{p}, t\right),
\end{aligned}
$$

is of the type $C_{m, l}\left(\sigma, M_{11}, M_{12}, M_{2}, \rho\right)$, if the following conditions are satisfied for some positive constants $\sigma, M_{11}, M_{12}, M_{2}$, and $\rho$ :

(i) $\boldsymbol{\omega} \cdot \boldsymbol{p}=\boldsymbol{\omega}_{0} \cdot \boldsymbol{p}_{0}+\boldsymbol{\omega}_{1} \cdot \boldsymbol{p}_{1}$ is bounded as

$$
|\boldsymbol{\omega} \cdot \boldsymbol{p}| \geq \sigma
$$

(ii) $t^{2} D$ can be extended to an analytic function in the domain $D_{2}(\rho):=\left.D(\rho)\right|_{\boldsymbol{q}=\mathbf{0}}$ in $\mathbb{C}^{n+1}$ and satisfies the inequality

$$
\left|t^{2} D\right| \leq \epsilon M_{11}
$$

Further, $t^{2} D$ is real if $(\boldsymbol{p}, 1 / t)$ are real.

(iii) $t^{l} E$ can be extended to an analytic function in the domain $D_{1}(\rho) \subset \mathbb{C}^{n+n_{1}+1}$ and satisfies the inequality

$$
\left|t^{l} E\right| \leq \epsilon^{l-1} M_{12}
$$

Further, $t^{l} E$ is periodic with respect to $\boldsymbol{q}_{1}$, real if $\left(\boldsymbol{q}_{1}, \boldsymbol{p}, 1 / t\right)$ is real, and satisfies

$$
\frac{1}{(2 \pi)^{n_{1}}} \int_{0}^{2 \pi} \cdots \int_{0}^{2 \pi} d^{n_{1}} \boldsymbol{q}_{1} t^{l} E=0 .
$$

(iv) $t^{m} B$ can be extended to an analytic function in the domain $D(\rho) \subset \mathbb{C}^{2 n+1}$ and satisfies the inequality

$$
\left|t^{m} B\right| \leq \epsilon^{2(m-1)} M_{2}
$$

Further, $t^{m} B$ is periodic with respect to $\boldsymbol{q}$, real if $(\boldsymbol{q}, \boldsymbol{p}, 1 / t)$ is real, and satisfies

$$
\frac{1}{(2 \pi)^{n_{0}}} \int_{0}^{2 \pi} \cdots \int_{0}^{2 \pi} d^{n_{0}} \boldsymbol{q}_{0} t^{m} B=0 .
$$

Since the interaction terms of the third degree of $\phi$ do not contribute $D, D$ begins with terms of order $\epsilon / t^{2}$.

First we show that we can make $\boldsymbol{q}_{0}$ depenent part $B$ small by the canonical transformation $S$. We say that the Hamiltonian is of the type $C_{m}^{2}\left(\sigma, M_{1}, M_{2}, \rho\right)$ if the conditions with $\epsilon$ replaced with $\epsilon^{2}$ in the definition of the type $C_{m}\left(\sigma, M_{1}, M_{2}, \rho\right)$ are satisfied. 
Proposition 5.1 Let $m$ be some positive interger, and consider the Hamitonian $H^{(m)}$ written in terms of fast canonical variables $\left(\boldsymbol{q}_{0}^{(m)}, \boldsymbol{p}_{0}^{(m)}\right)$ and slow canonical variables $\left(\boldsymbol{q}_{1}^{(m)}, \boldsymbol{p}_{1}^{(m)}\right)$ as

$$
H^{(m)}=\frac{2}{\epsilon^{2}}\left(\boldsymbol{\omega}_{0} \cdot \boldsymbol{p}_{0}^{(m)}+\boldsymbol{\omega}_{1} \cdot \boldsymbol{p}_{1}^{(m)}\right)^{1 / 2}+A_{m}\left(\boldsymbol{q}_{1}^{(m)}, \boldsymbol{p}^{(m)}, t\right)+B_{m}\left(\boldsymbol{q}^{(m)}, \boldsymbol{p}^{(m)}, t\right) .
$$

Suppose that $\boldsymbol{\omega}$ is of the class $D_{0}(d, C)$ and that this Hamiltonian is of the type $C_{m}^{2}\left(\sigma_{m}, M_{1}^{(m)}, M_{2}^{(m)}, \rho_{m}\right)$. Then, for any $\delta>0$, there exists $\epsilon_{0}>0$ such that, for an arbitrary $\epsilon$ satisfying

$$
0<\epsilon<\epsilon_{0},
$$

there exists a function $S_{m}(\boldsymbol{q}, \boldsymbol{p}, t)$ satisfying the following conditions:

(i) $S_{m}$ is periodic with respect to $\boldsymbol{q}$ and real if $(\boldsymbol{q}, \boldsymbol{p}, t)$ is real.

(ii) $t^{m} S_{m}$ can be extended to an analytic function in the multi-dimensional complex domain $D\left(\rho_{m+1}\right)$, where $\rho_{m+1}=\rho_{m}-\delta$, and in this domain, satisfies the inequality

$$
\left|t^{m} S_{m}\right| \leq \epsilon^{2 m} L_{1}^{(m)}
$$

for some positive constant $L_{1}^{(m)}$.

(iii) Let $H^{(m+1)}\left(\boldsymbol{q}^{(m+1)}, \boldsymbol{p}^{(m+1)}, t\right)$ be a Hamitonian obtained from $H^{(m)}$ by the canonical transformation generated by $S_{m}\left(\boldsymbol{q}^{(m)}, \boldsymbol{p}^{(m+1)}, t\right)$ :

$$
\begin{aligned}
& \boldsymbol{p}^{(m)}=\boldsymbol{p}^{(m+1)}+\frac{\partial S_{m}}{\partial \boldsymbol{q}^{(m)}} \\
& \boldsymbol{q}^{(m+1)}=\boldsymbol{q}^{(m)}+\frac{\partial S_{m}}{\partial \boldsymbol{p}^{(m+1)}} \\
& H^{(m+1)}=H^{(m)}+\frac{\partial S_{m}}{\partial t} \\
& \quad=\frac{2}{\epsilon^{2}}\left(\boldsymbol{\omega} \cdot \boldsymbol{p}^{(m+1)}\right)^{1 / 2}+A_{m+1}\left(\boldsymbol{q}_{1}^{(m+1)}, \boldsymbol{p}^{(m+1)}, t\right)+B_{m+1}\left(\boldsymbol{q}^{(m+1)}, \boldsymbol{p}^{(m+1)}\right.
\end{aligned}
$$

Then, $H^{(m+1)}$ is of the type $C_{m+1}^{2}\left(\sigma_{m+1}, M_{1}^{(m+1)}, M_{2}^{(m+1)}, \rho_{m+1}\right)$ for some positive constants $\sigma_{m+1}, M_{1}^{(m+1)}$, and $M_{2}^{(m+1)}$, and the change of the $A$-term in the Hamiltonians satisfies the inequality

$$
\left|t^{m+1}\left\{A_{m+1}\left(\boldsymbol{q}_{1}, \boldsymbol{p}, t\right)-A_{m}\left(\boldsymbol{q}_{1}, \boldsymbol{p}, t\right)\right\}\right| \leq \frac{\epsilon^{2 m}}{2} M_{2}^{(m+1)},
$$

for $\left(\boldsymbol{q}_{1}, \boldsymbol{p}, 1 / t\right) \in D_{1}\left(\rho_{m+1}\right)$.

The proof is obtained by replacing $\epsilon$ with $\epsilon^{2}$ in the proof of the Proposition 4.1.

We show that the fast action variables $\boldsymbol{p}_{0}$ are perpetually stable and oscillate around the initial values with amplitudes of order $\epsilon^{2}$ and with frequencies of order $1 / \epsilon^{2}$.

Proposition 5.2 There exists some constant $C_{0}$ such that

$$
\left|\boldsymbol{p}_{0}^{(1)}-\boldsymbol{p}_{0}^{(1)}(1)\right| \leq \epsilon^{2} C_{0} .
$$


proof We consider the transformed Hamiltonian given by

$$
H^{(2)}=\frac{2}{\epsilon^{2}}\left(\boldsymbol{\omega} \cdot \boldsymbol{p}^{(2)}\right)^{1 / 2}+A_{2}+B_{2}
$$

where

$$
\begin{aligned}
\left|t A_{2}\right| & \leq M_{1}^{(2)} \\
\left|t^{2} B_{2}\right| & \leq \epsilon^{2} M_{2}^{(2)}
\end{aligned}
$$

which gives

$$
\frac{d \boldsymbol{p}_{0}^{(2)}}{d t}=-\frac{\partial B_{2}}{\partial \boldsymbol{q}_{0}^{(2)}}
$$

We obtain

$$
\left|\boldsymbol{p}_{0}^{(2)}-\boldsymbol{p}_{0}^{(2)}(1)\right| \leq \int_{1} d t \frac{\epsilon^{2}}{t^{2}} \frac{M_{2}^{(2)}}{\delta} \leq \epsilon^{2} \frac{M_{2}^{(2)}}{\delta} .
$$

By using the transformation law

$$
\left|\boldsymbol{p}^{(1)}-\boldsymbol{p}^{(2)}\right| \leq\left|\frac{\partial S_{1}}{\partial \boldsymbol{q}^{(1)}}\right| \leq \frac{\epsilon^{2}}{t} \frac{L_{1}^{(1)}}{\delta},
$$

we obtain

$$
\begin{aligned}
& \left|\boldsymbol{p}_{0}^{(1)}-\boldsymbol{p}_{0}^{(1)}(1)\right| \\
& \leq\left|\boldsymbol{p}_{0}^{(1)}-\boldsymbol{p}_{0}^{(2)}\right|+\left|\boldsymbol{p}_{0}^{(2)}-\boldsymbol{p}_{0}^{(2)}(1)\right|+\left|\boldsymbol{p}_{0}^{(2)}(1)-\boldsymbol{p}_{0}^{(1)}(1)\right| \\
& \leq \epsilon^{2} \frac{L_{1}^{(1)}}{\delta}+\epsilon^{2} \frac{M_{2}^{(2)}}{\delta}+\epsilon^{2} \frac{L_{1}^{(1)}}{\delta}
\end{aligned}
$$

which completes the proof.

Next we show that we can make $\boldsymbol{q}_{1}$ dependent part $E$ small by the canonical transformation $T$.

Proposition 5.3 Let $m, l$ be some positive intergers, and consider the Hamitonian $H^{(m, l)}$ written in terms of fast canonical variables $\left(\boldsymbol{q}_{0}^{(m, l)}, \boldsymbol{p}_{0}^{(m, l)}\right)$ and slow canonical variables $\left(\boldsymbol{q}_{1}^{(m, l)}, \boldsymbol{p}_{1}^{(m, l)}\right)$ as

$H^{(m, l)}=\frac{2}{\epsilon^{2}}\left(\boldsymbol{\omega}_{0} \cdot \boldsymbol{p}_{0}^{(m, l)}+\boldsymbol{\omega}_{1} \cdot \boldsymbol{p}_{1}^{(m, l)}\right)^{1 / 2}+D_{m, l}\left(\boldsymbol{p}^{(m, l)}, t\right)+E_{m, l}\left(\boldsymbol{q}_{1}^{(m, l)}, \boldsymbol{p}^{(m, l)}, t\right)+B_{m, l}\left(\boldsymbol{q}^{(m, l)}, \boldsymbol{p}^{(m, l)}, t\right)$.

Suppose that $\boldsymbol{\omega}$ is of the class $D(d, \epsilon C)$ and that this Hamiltonian is of the type $C_{m, l}\left(\sigma_{m, l}, M_{11}^{(m, l)}, M_{12}^{(m, l)}, M_{2}^{(m, l)}, \rho_{m, l}\right)$. Then, for any $\delta>0$, there exists $\epsilon_{0}>0$ such that, for an arbitrary $\epsilon$ satisfying

$$
0<\epsilon<\epsilon_{0},
$$

there exists a function $T_{m, l}\left(\boldsymbol{q}_{1}, \boldsymbol{p}, t\right)$ satisfying the following conditions:

(i) $T_{m, l}$ is periodic with respect to $\boldsymbol{q}_{1}$ and real if $\left(\boldsymbol{q}_{1}, \boldsymbol{p}, t\right)$ is real. 
(ii) $t^{l} T_{m, l}$ can be extended to an analytic function in the multi-dimensional complex domain $D_{1}\left(\rho_{m, l+1}\right)$, where $\rho_{m, l+1}=\rho_{m, l}-\delta$, and in this domain, satisfies the inequality

$$
\left|t^{l} T_{m, l}\right| \leq \epsilon^{l} L_{2}^{(m, l)}
$$

for some positive constant $L_{2}^{(m, l)}$.

(iii) Let $H^{(m, l+1)}\left(\boldsymbol{q}^{(m, l+1)}, \boldsymbol{p}^{(m, l+1)}, t\right)$ be a Hamitonian obtained from $H^{(m, l)}$ by the canonical transformation generated by $T_{m, l}\left(\boldsymbol{q}_{1}^{(m, l)}, \boldsymbol{p}^{(m, l+1)}, t\right)$ :

$$
\begin{aligned}
& \boldsymbol{p}_{0}^{(m, l)}=\boldsymbol{p}_{0}^{(m, l+1)} \\
& \boldsymbol{p}_{1}^{(m, l)}=\boldsymbol{p}_{1}^{(m, l+1)}+\frac{\partial T_{m, l}}{\partial \boldsymbol{q}_{1}^{(m, l)}} \\
& \boldsymbol{q}^{(m, l+1)}=\boldsymbol{q}^{(m, l)}+\frac{\partial T_{m, l}}{\partial \boldsymbol{p}^{(m, l+1)}} \\
& H^{(m, l+1)}=H^{(m, l)}+\frac{\partial T_{m, l}}{\partial t} \\
& =\frac{2}{\epsilon^{2}}\left(\boldsymbol{\omega} \cdot \boldsymbol{p}^{(m, l+1)}\right)^{1 / 2}+D_{m, l+1}\left(\boldsymbol{p}^{(m, l+1)}, t\right)+E_{m, l+1}\left(\boldsymbol{q}_{1}^{(m, l+1)}, \boldsymbol{p}^{(m, l+1)}, t\right) \\
& \quad+B_{m, l+1}\left(\boldsymbol{q}^{(m, l+1)}, \boldsymbol{p}^{(m, l+1)}, t\right)
\end{aligned}
$$

Then, $H^{(m, l+1)}$ is of the type $C_{m, l+1}\left(\sigma_{m, l+1}, M_{11}^{(m, l+1)}, M_{12}^{(m, l+1)}, M_{2}^{(m, l+1)}, \rho_{m, l+1}\right)$ for some positive constants $\sigma_{m, l+1}, M_{11}^{(m, l+1)}, M_{12}^{(m, l+1)}$, and $M_{2}^{(m, l+1)}$, and the change of the $D$-term in the Hamiltonians satisfies the inequality

$$
\left|t^{l+1}\left\{D_{m, l+1}(\boldsymbol{p}, t)-D_{m, l}(\boldsymbol{p}, t)\right\}\right| \leq \frac{\epsilon^{l}}{2} M_{12}^{(m, l+1)},
$$

for $(\boldsymbol{p}, 1 / t) \in D_{2}\left(\rho_{m, l+1}\right)$.

(For the proof, see the appendix $C$ ).

We show that the slow action variables $\boldsymbol{p}_{1}$ are perpetually stable and oscillate around the initial values with amplitudes of order $\epsilon$ and with frequencies of order $1 / \epsilon$. Notice that $T$ is independent of $\boldsymbol{q}_{0}$.

Proposition 5.4 There exists some constant $C_{1}$ such that

$$
\left|\boldsymbol{p}_{1}^{(1)}-\boldsymbol{p}_{1}^{(1)}(1)\right| \leq \epsilon C_{1} .
$$

proof We consider the transformed Hamiltonian $H^{(m, l)}(m=2, l=2)$ given by

$$
H^{(2,2)}=\frac{2}{\epsilon^{2}}\left(\boldsymbol{\omega} \cdot \boldsymbol{p}^{(2,2)}\right)^{1 / 2}+D_{22}+E_{22}+B_{22},
$$

where

$$
\begin{aligned}
& \left|t^{2} D_{22}\right| \leq \epsilon M_{11}^{(2,2)} \\
& \left|t^{2} E_{22}\right| \leq \epsilon M_{12}^{(2,2)} \\
& \left|t^{2} B_{22}\right| \leq \epsilon^{2} M_{2}^{(2,2)}
\end{aligned}
$$


The evolution equations for the slow action variables $\boldsymbol{p}_{1}$ are given by

$$
\frac{d \boldsymbol{p}_{1}^{(2,2)}}{d t}=-\frac{\partial E_{22}}{\partial \boldsymbol{q}_{1}^{(2,2)}}-\frac{\partial B_{22}}{\partial \boldsymbol{q}_{1}^{(2,2)}} \sim \frac{\epsilon}{t^{2}},
$$

which yields

$$
\left|\boldsymbol{p}_{1}^{(2,2)}-\boldsymbol{p}_{1}^{(2,2)}(1)\right| \leq \epsilon C,
$$

for some positive constant $C$.

$$
\begin{aligned}
& \left|\boldsymbol{p}_{1}^{(1,1)}(t)-\boldsymbol{p}_{1}^{(1,1)}(1)\right| \\
\leq & \left|\boldsymbol{p}_{1}^{(1,1)}(t)-\boldsymbol{p}_{1}^{(2,2)}(t)\right|+\left|\boldsymbol{p}_{1}^{(2,2)}(t)-\boldsymbol{p}_{1}^{(2,2)}(1)\right|+\left|\boldsymbol{p}_{1}^{(2,2)}(1)-\boldsymbol{p}_{1}^{(1,1)}(1)\right| \\
\leq & \epsilon C_{1} .
\end{aligned}
$$

We investigate the dynamical behavior of the cosmological perturbations. We obtain the Hamiltonian $H^{(3,3)}$ of the type $C_{3,3}\left(\sigma_{3,3}, M_{11}^{(3,3)}, M_{12}^{(3,3)}, M_{2}^{(3,3)}, \rho_{3,3}\right)$ by carrying out the canonical transformations in the order $S_{1}, S_{2}, T_{3,1}, T_{3,2}$.

Later we omit the superscript $(3,3)$ and constant coefficients of order 1 .

From now on we assume that

$$
\left|\delta \boldsymbol{q}_{0}(1)\right| \sim\left|\delta \boldsymbol{p}_{0}(1)\right| \sim\left|\delta \boldsymbol{q}_{1}(1)\right| \sim\left|\delta \boldsymbol{p}_{1}(1)\right| \sim 1
$$

since in the linear perturbation, the scale of the perturbation variables is arbitrary. Then we obtain the proposition below.

Proposition 5.5 The transformed perturbation variables are estimated as

$$
\begin{aligned}
& \left|\delta \boldsymbol{p}_{0}-\delta \boldsymbol{p}_{0}(1)\right| \leq \epsilon^{2} \\
& \left|\delta \boldsymbol{p}_{1}-\delta \boldsymbol{p}_{1}(1)\right| \leq \epsilon \\
& \delta \boldsymbol{q}_{0}=-\frac{1}{2} \frac{\boldsymbol{\omega}_{0}}{\epsilon^{2}}(t-1) \frac{1}{(\boldsymbol{\omega} \cdot \boldsymbol{p}(1))^{3 / 2}}\left(\boldsymbol{\omega}_{0} \cdot \delta \boldsymbol{p}_{0}(1)+\boldsymbol{\omega}_{1} \cdot \delta \boldsymbol{p}_{1}(1)\right)+\boldsymbol{R}_{0} \\
& \delta \boldsymbol{q}_{1}=-\frac{1}{2} \frac{\boldsymbol{\omega}_{1}}{\epsilon^{2}}(t-1) \frac{1}{(\boldsymbol{\omega} \cdot \boldsymbol{p}(1))^{3 / 2}}\left(\boldsymbol{\omega}_{0} \cdot \delta \boldsymbol{p}_{0}(1)+\boldsymbol{\omega}_{1} \cdot \delta \boldsymbol{p}_{1}(1)\right)+\boldsymbol{R}_{1}
\end{aligned}
$$

where the residual part $\boldsymbol{R}_{0}, \boldsymbol{R}_{1}$ is bounded as

$$
\begin{aligned}
& \left|\boldsymbol{R}_{0}\right| \leq C_{0}(t-1) \\
& \left|\boldsymbol{R}_{1}\right| \leq \epsilon C_{1}(t-1)
\end{aligned}
$$

for some positive constants $C_{0}, C_{1}$.

For the proof see the appendix (subsection D.1).

We have evaluated the canonically transformed variables $\delta \boldsymbol{q}^{(3,3)}, \delta \boldsymbol{p}^{(3,3)}$. Then we evaluate the difference between such transformed variables and the original variables $\delta \boldsymbol{q}^{(1,1)}$, $\delta \boldsymbol{p}^{(1,1)}$. 


\section{Proposition 5.6}

$$
\begin{aligned}
& \left|\delta \boldsymbol{q}^{(1,1)}-\delta \boldsymbol{q}^{(3,3)}\right| \leq 1 \\
& \left|\delta \boldsymbol{p}^{(1,1)}-\delta \boldsymbol{p}^{(3,3)}\right| \leq 1 .
\end{aligned}
$$

For the proof see the appendix (subsection D.2).

According to propositions 5.5, 5.6, for $t \geq 1 / \epsilon$, the Bardeen parameter $\zeta$ stays constant in a good accuracy.

\section{$\S 6$ Discussion}

In this paper we have constructed the method for analyzing the resonance phenomena of the Hamiltonian system obtained from the multiple oscillatory scalar fields in the expanding universe. We have shown that the perturbations including the Bardeen parameter can grow when the resonant interactions between the homogeneous modes exist. If the truncated Hamiltonian system has hyperbolic fixed points in the phase space of the slow variables, the perturbations grow at the speed of a power of $t$ in the three leg interaction systems, while the growth of perturbations is bounded from above in no less than four leg interaction systems. In the models where $\boldsymbol{\omega}_{1}=0$, we have found the network constructed by the heteroclinic orbits around which perturbations are unstable. On this network, the orbits become irregular, unperiodic, complicated and probabilistic. In order to evaluate the amplitude of the Bardeen parameter which is directly related the cosmic structure formation, we must calculate the measure occupied by the stochastic network in the whole phase space of the homogeneous modes.

Recentlty the relaxation in reheating is investigated numerically and is interpreted in terms of the turbulence phenomena of the dynamical system of large number of degrees of freedom [14]. In order to understand the turbulence phenomena, our fixed point analysis of the Hamiltonian system may be useful.

\section{Acknowledgments}

The author would like to thank Professor H. Kodama for reading his manuscript carefully, helping him improve his English and giving him useful comments The author would also like to thank Professor V.I.Arnold for his writing the excellent textbooks and/or reviews [15] ], from which the author learned quite a lot about the Hamiltonian dynamical system.

\section{§A Appendix; Proof of Proposition 4.1}

We consider the canonical transformation induced by the generating function given by

$$
S_{m}\left(\boldsymbol{p}^{(m+1)}, \boldsymbol{q}^{(m)}, t\right)=\sum_{\boldsymbol{k}_{0} \neq \mathbf{0}} S_{\boldsymbol{k}}\left(\boldsymbol{p}^{(m+1)}, t\right) e^{i \boldsymbol{k} \cdot \boldsymbol{q}^{(m)}}
$$


where $\boldsymbol{k} \cdot \boldsymbol{q}^{(m)}=\boldsymbol{k}_{0} \cdot \boldsymbol{q}_{0}^{(m)}+\boldsymbol{k}_{1} \cdot \boldsymbol{q}_{1}^{(m)}$ and the sum is taken over $\boldsymbol{k}$ such that $\boldsymbol{k}_{0} \neq \mathbf{0}$. $B_{m}$ is decomposed as

$$
B_{m}\left(\boldsymbol{p}^{(m+1)}, \boldsymbol{q}^{(m)}, t\right)=\sum_{\boldsymbol{k}_{0} \neq \mathbf{0}} b_{\boldsymbol{k}}\left(\boldsymbol{p}^{(m+1)}, t\right) e^{i \boldsymbol{k} \cdot \boldsymbol{q}^{(m)}},
$$

where the sum is taken over $\boldsymbol{k}$ satisfying $\boldsymbol{k}_{0} \neq \mathbf{0}$.

The transformed Hamiltonian is

$$
\begin{aligned}
& H^{(m+1)}=H^{(m)}+\frac{\partial S_{m}}{\partial t} \\
& =\frac{2}{\epsilon}\left(\boldsymbol{\omega} \cdot \boldsymbol{p}^{(m+1)}\right)^{1 / 2}+\frac{1}{\epsilon} \frac{1}{\left(\boldsymbol{\omega} \cdot \boldsymbol{p}^{(m+1)}\right)^{1 / 2}} \boldsymbol{\omega} \cdot \frac{\partial S_{m}\left(\boldsymbol{q}^{(m)}, \boldsymbol{p}^{(m+1)}, t\right)}{\partial \boldsymbol{q}^{(m)}}+R_{1} \\
& +A_{m}\left(\boldsymbol{q}_{1}^{(m+1)}, \boldsymbol{p}^{(m+1)}, t\right)+R_{2} \\
& +B_{m}\left(\boldsymbol{q}^{(m)}, \boldsymbol{p}^{(m+1)}, t\right)+R_{3}+\frac{\partial S_{m}\left(\boldsymbol{q}^{(m)}, \boldsymbol{p}^{(m+1)}, t\right)}{\partial t}
\end{aligned}
$$

where $R_{1}, \cdots R_{3}$ are defined as

$$
\begin{aligned}
& R_{1}=\frac{2}{\epsilon}\left(\boldsymbol{\omega} \cdot \boldsymbol{p}^{(m)}\right)^{1 / 2}-\frac{2}{\epsilon}\left(\boldsymbol{\omega} \cdot \boldsymbol{p}^{(m+1)}\right)^{1 / 2}-\frac{1}{\epsilon} \frac{\boldsymbol{\omega}}{\left(\boldsymbol{\omega} \cdot \boldsymbol{p}^{(m+1)}\right)^{1 / 2}} \cdot\left(\boldsymbol{p}^{(m)}-\boldsymbol{p}^{(m+1)}\right), \\
& R_{2}=A_{m}\left(\boldsymbol{q}_{1}^{(m)}, \boldsymbol{p}^{(m)}, t\right)-A_{m}\left(\boldsymbol{q}_{1}^{(m+1)}, \boldsymbol{p}^{(m+1)}, t\right) \\
& R_{3}=B_{m}\left(\boldsymbol{q}^{(m)}, \boldsymbol{p}^{(m)}, t\right)-B_{m}\left(\boldsymbol{q}^{(m)}, \boldsymbol{p}^{(m+1)}, t\right)
\end{aligned}
$$

We determine the generating function $S_{m}$ so that the leading term depending on the fast angle variables $\boldsymbol{q}_{0}^{(m)}$ can be eliminated;

$$
\frac{1}{\epsilon} \frac{1}{\left(\boldsymbol{\omega} \cdot \boldsymbol{p}^{(m+1)}\right)^{1 / 2}} \boldsymbol{\omega} \cdot \frac{\partial S_{m}\left(\boldsymbol{q}^{(m)}, \boldsymbol{p}^{(m+1)}, t\right)}{\partial \boldsymbol{q}^{(m)}}=-B_{m}\left(\boldsymbol{q}^{(m)}, \boldsymbol{p}^{(m+1)}, t\right) .
$$

By comparing the Fourier components in the both hand sides, we obtain

$$
S_{\boldsymbol{k}}\left(\boldsymbol{p}^{(m+1)}, t\right)=i \epsilon\left(\boldsymbol{\omega} \cdot \boldsymbol{p}^{(m+1)}\right)^{1 / 2} \frac{1}{(\boldsymbol{\omega} \cdot \boldsymbol{k})} b_{\boldsymbol{k}}\left(\boldsymbol{p}^{(m+1)}, t\right)
$$

Before we prove the analyticity and evaluate the upper bound of $S_{m}\left(\boldsymbol{q}^{(m)}, \boldsymbol{p}^{(m+1)}, t\right)$, we use the following lemma.

\section{Lemma}

As for the Fourier series

$$
F(\boldsymbol{q})=\sum_{\boldsymbol{k}} F_{\boldsymbol{k}} e^{i \boldsymbol{k} \cdot \boldsymbol{q}}
$$

where

$$
\boldsymbol{k} \cdot \boldsymbol{q}=\sum_{i=1}^{n} k_{i} q_{i}
$$

(1) If $F(\boldsymbol{q})$ is analytic and satisfies

$$
|F(\boldsymbol{q})| \leq C
$$


on the domain $|\operatorname{Im} \boldsymbol{q}| \leq \rho$, then

$$
\left|F_{k}\right| \leq C e^{-|\boldsymbol{k}| \rho},
$$

where

$$
|\boldsymbol{k}|=\left|k_{1}\right|+\cdots+\left|k_{n}\right| .
$$

(2) If on the domain $|\operatorname{Im} \boldsymbol{q}| \leq \rho, F_{\boldsymbol{k}}$ satrisfies

$$
\left|F_{k}\right| \leq C e^{-|k| \rho},
$$

then $F(\boldsymbol{q})$ is analytic on the domain $|\operatorname{Im} \boldsymbol{q}| \leq \rho$, and for an arbitrary $\delta(0<\delta<\rho)$, on the domain $|\operatorname{Im} \boldsymbol{q}| \leq \rho-\delta$

$$
|F(\boldsymbol{q})| \leq \frac{4^{n} C}{\delta^{n}},
$$

where we assume $\delta \leq 3$.

Proof of Lemma

(1) The Fourier coefficients $F_{\boldsymbol{k}}$ are given by

$$
F_{\boldsymbol{k}}=\frac{1}{(2 \pi)^{n}} \int_{0}^{2 \pi} \cdots \int_{0}^{2 \pi} d^{n} \boldsymbol{q} e^{-i \boldsymbol{k} \cdot \boldsymbol{q}} F(\boldsymbol{q}) .
$$

By Cauchy's theorem, the path of integration in the above integral can be shifted to $q_{i}=x_{i} \pm i \rho, 0 \leq x_{i} \leq 2 \pi$ where we choose the sign equal to $-k_{i}$, we get

$$
\left|F_{k}\right| \leq C e^{-|\boldsymbol{k}| \rho} .
$$

(2) For an arbitrary positive $\delta,(0<\delta<\rho)$, on the domain $|\operatorname{Im} \boldsymbol{q}| \leq \rho-\delta$,

$$
\left|e^{i \boldsymbol{k} \cdot \boldsymbol{q}}\right| \leq e^{|\boldsymbol{k}|(\rho-\delta)} .
$$

On the domain $|\operatorname{Im} \boldsymbol{q}| \leq \rho-\delta$

$$
\begin{aligned}
|F| & \leq \sum_{\boldsymbol{k}}\left|F_{\boldsymbol{k}}\right|\left|e^{i \boldsymbol{k} \cdot \boldsymbol{q}}\right| \\
& \leq \sum_{\boldsymbol{k}} C e^{-|\boldsymbol{k}| \rho} e^{|\boldsymbol{k}|(\rho-\delta)} \\
& =\sum_{\boldsymbol{k}} C e^{-|\boldsymbol{k}| \delta} \\
& =C\left(1+2 \sum_{k>0} e^{-k \delta}\right)^{n} \\
& =C\left(\frac{1+e^{-\delta}}{1-e^{-\delta}}\right)^{n} \\
& <C\left(\frac{4}{\delta}\right)^{n},
\end{aligned}
$$

where we use the inequality

$$
\frac{1+e^{-\delta}}{1-e^{-\delta}}<\left(\frac{4}{\delta}\right)
$$


for $0<\delta \leq 3$. Namely on the domain $|\operatorname{Im} \boldsymbol{q}| \leq \rho-\delta, F(\boldsymbol{q})$ converges uniformly and absolutely, therefore $F(\boldsymbol{q})$ is analytic. Since $\delta$ is arbitrary, $F(\boldsymbol{q})$ is analytic on the domain $|\operatorname{Im} \boldsymbol{q}| \leq \rho$.

Proof End of Lemma

We evaluate the right hand side of (A.8). For arbitrary $\boldsymbol{p}^{(m+1)} \in D\left(\rho_{m}\right)$, we put $\tau_{m}$ as the maximum value of $\boldsymbol{\omega} \cdot \boldsymbol{p}^{(m+1)}$;

$$
\sigma_{m} \leq \boldsymbol{\omega} \cdot \boldsymbol{p}^{(m+1)} \leq \tau_{m}
$$

Since $t^{m} B_{m}$ is analytic and bounded by $\epsilon^{m-1} M_{2}^{(m)}$ on the domain $D\left(\rho_{m}\right)$,

$$
\left|t^{m} b_{\boldsymbol{k}}\left(\boldsymbol{p}^{(m+1)}, t\right)\right| \leq \epsilon^{m-1} M_{2}^{(m)} e^{-|\boldsymbol{k}| \rho_{m}}
$$

As $|\boldsymbol{k}|$ increases, while $b_{\boldsymbol{k}}$ decay exponentially, the contribution from the denominator grows like power;

$$
\frac{1}{(\boldsymbol{\omega} \cdot \boldsymbol{k})} \leq \frac{|\boldsymbol{k}|^{d}}{C} .
$$

The exponential decay defeats the power grow. We use the inequality

$$
|\boldsymbol{k}|^{d} \leq e^{|\boldsymbol{k}| \delta}\left(\frac{d}{e}\right)^{d} \frac{1}{\delta^{d}}
$$

for an arbitrary positive $\delta$. The proof is as follows. $f(x)=x-d \ln x$ has a minimum at $x=d$. Therefore

$$
\frac{e^{x}}{x^{d}} \geq \frac{e^{d}}{d^{d}}
$$

For $x=|\boldsymbol{k}| \delta$, we obtain this inequality. We evaluate the Fourier components $S_{\boldsymbol{k}}$ as

$$
\begin{aligned}
\left|t^{m} S_{\boldsymbol{k}}\left(\boldsymbol{p}^{(m+1)}, t\right)\right| & \leq \epsilon \tau_{m}^{1 / 2} \frac{|\boldsymbol{k}|^{d}}{C} \epsilon^{m-1} M_{2}^{(m)} \exp \left(-|\boldsymbol{k}| \rho_{m}\right) \\
& \leq \epsilon^{m} \tau_{m}^{1 / 2} \frac{M_{2}^{(m)}}{C}\left(\frac{d}{e}\right)^{d} \frac{1}{\delta^{d}} \exp \left\{-|\boldsymbol{k}|\left(\rho_{m}-\delta\right)\right\} .
\end{aligned}
$$

Therefore $t^{m} S_{m}$ is analytic on the domain $D\left(\rho_{m}\right)$ and bounded as

$$
\left|t^{m} S_{m}\right| \leq \epsilon^{m} L_{1}^{(m)}
$$

where

$$
L_{1}^{(m)}=\tau_{m}^{1 / 2} \frac{M_{2}^{(m)}}{C}\left(\frac{d}{e}\right)^{d} \frac{4^{n}}{\delta^{n+d}},
$$

on the domain $D\left(\rho_{m}-2 \delta\right)$ for an arbitrary positive $\delta \leq 3$. We put $\rho_{m+1}=\rho_{m}-3 \delta$ and on the domain $D\left(\rho_{m+1}\right)$

$$
\begin{aligned}
\left|\boldsymbol{p}^{(m)}-\boldsymbol{p}^{(m+1)}\right| & \leq\left|\frac{\partial S_{m}}{\partial \boldsymbol{q}^{(m)}}\right| \leq \epsilon^{m} \frac{L_{1}^{(m)}}{\delta}\left(1+\rho_{m+1}\right)^{m} \\
\left|\boldsymbol{q}^{(m+1)}-\boldsymbol{q}^{(m)}\right| & \leq\left|\frac{\partial S_{m}}{\partial \boldsymbol{p}^{(m+1)}}\right| \leq \epsilon^{m} \frac{L_{1}^{(m)}}{\delta}\left(1+\rho_{m+1}\right)^{m} .
\end{aligned}
$$


Therefore if $\epsilon$ satisfies

$$
\epsilon^{m} \frac{L_{1}^{(m)}}{\delta}\left(1+\rho_{m+1}\right)^{m} \leq 3 \delta,
$$

when $\left(\boldsymbol{p}^{(m+1)}, \boldsymbol{q}^{(m+1)}\right) \in D\left(\rho_{m+1}\right),\left(\boldsymbol{p}^{(m)}, \boldsymbol{q}^{(m)}\right) \in D\left(\rho_{m}\right)$.

Next on the domain $D\left(\rho_{m+1}\right)$, we evaluate the residual part $R$ defined as

$$
R=R_{1}+R_{2}+R_{3}+\frac{\partial S_{m}\left(\boldsymbol{q}^{(m)}, \boldsymbol{p}^{(m+1)}, t\right)}{\partial t} .
$$

For evaluation, we use the Taylor expansion;

$$
\left|F(1)-F(0)-\sum_{k=1}^{n-1} \frac{1}{k !} F^{(k)}(0)\right| \leq \frac{1}{n !}\left[F^{(n)}\right],
$$

where $[A]$ means the maximum value of $|A|$. As the function $F(\xi)$, we take

$$
F(\xi)=f\left(\boldsymbol{x}_{0}+\xi\left(\boldsymbol{x}-\boldsymbol{x}_{0}\right)\right),
$$

where $\boldsymbol{x}$ is the multidimensional vector.

$$
\begin{aligned}
& F^{(1)}(0)=\left.\frac{\partial f}{\partial \boldsymbol{x}^{i}}\right|_{0}\left(\boldsymbol{x}-\boldsymbol{x}_{0}\right)^{i}, \\
& F^{(2)}(0)=\left.\frac{\partial^{2} f}{\partial \boldsymbol{x}^{i} \partial \boldsymbol{x}^{j}}\right|_{0}\left(\boldsymbol{x}-\boldsymbol{x}_{0}\right)^{i}\left(\boldsymbol{x}-\boldsymbol{x}_{0}\right)^{j} .
\end{aligned}
$$

The residual part is

$$
\frac{1}{n !}\left[F^{(n)}\right]=\frac{1}{n !}\left[\frac{\partial^{n} F}{\partial \boldsymbol{x}^{i_{1}} \cdots \partial \boldsymbol{x}^{i_{n}}}\left(\boldsymbol{x}-\boldsymbol{x}_{0}\right)^{i_{1}} \cdots\left(\boldsymbol{x}-\boldsymbol{x}_{0}\right)^{i_{n}}\right] .
$$

As for $R_{1}$,

$$
\left|R_{1}\right| \leq\left[-\frac{1}{4 \epsilon} \frac{\boldsymbol{\omega}_{i} \boldsymbol{\omega}_{j}}{(\boldsymbol{\omega} \cdot \boldsymbol{p})^{3 / 2}}\left(\boldsymbol{p}^{(m)}-\boldsymbol{p}^{(m+1)}\right)^{i}\left(\boldsymbol{p}^{(m)}-\boldsymbol{p}^{(m+1)}\right)^{j}\right] .
$$

Then we evaluate

$$
\left|t^{2 m} R_{1}\right| \leq \frac{1}{4 \epsilon} \frac{1}{\sigma_{m}^{3 / 2}}\left\{\boldsymbol{\omega} n \frac{\epsilon^{m} L_{1}^{(m)}}{\delta}\right\}^{2}
$$

As for $R_{2}$,

$$
\left|R_{2}\right| \leq\left[\frac{\partial A_{m}}{\partial \boldsymbol{q}_{1}} \cdot\left(\boldsymbol{q}_{1}^{(m)}-\boldsymbol{q}_{1}^{(m+1)}\right)+\frac{\partial A_{m}}{\partial \boldsymbol{p}} \cdot\left(\boldsymbol{p}^{(m)}-\boldsymbol{p}^{(m+1)}\right)\right] .
$$

Then we evaluate

$$
\left|t^{m+1} R_{2}\right| \leq 2 n \frac{M_{1}^{(m)}}{\delta} \epsilon^{m} \frac{L_{1}^{(m)}}{\delta} .
$$

As for $R_{3}$,

$$
\left|R_{3}\right| \leq\left[\frac{\partial B_{m}\left(\boldsymbol{q}^{(m)}, \boldsymbol{p}, t\right)}{\partial \boldsymbol{p}} \cdot\left(\boldsymbol{p}^{(m)}-\boldsymbol{p}^{(m+1)}\right)\right] .
$$


Then we evaluate

$$
\left|t^{2 m} R_{3}\right| \leq n \epsilon^{m-1} \frac{M_{2}^{(m)}}{\delta} \epsilon^{m} \frac{L_{1}^{(m)}}{\delta} .
$$

In addition,

$$
\left|t^{m+1} \frac{\partial S_{m}}{\partial t}\right| \leq \epsilon^{m} L_{1}^{(m)}\left(m+\frac{1+\rho_{m+1}}{\delta}\right) .
$$

Therefore we obtain

$$
\left|t^{m+1} R\right| \leq \epsilon^{m} M
$$

where

$$
\begin{aligned}
M= & \epsilon^{m-1}\left(1+\rho_{m+1}\right)^{m-1} \frac{1}{4 \sigma_{m}^{3 / 2}}\left[\boldsymbol{\omega} n \frac{L_{1}^{(m)}}{\delta}\right]^{2}+2 n \frac{M_{1}^{(m)} L_{1}^{(m)}}{\delta^{2}} \\
& +\epsilon^{m-1} n\left(1+\rho_{m+1}\right)^{m-1} \frac{M_{2}^{(m)} L_{1}^{(m)}}{\delta^{2}}+L_{1}^{(m)}\left(m+\frac{1+\rho_{m+1}}{\delta}\right) .
\end{aligned}
$$

We decompose $R$ into the slowly varying part $R_{s}$ and the fast varying part $R_{f}$ as

$$
R=R_{s}+R_{f},
$$

where

$$
\begin{aligned}
& R_{s}=\sum_{\boldsymbol{k}_{0}=\mathbf{0}} R_{\mathbf{0} \boldsymbol{k}_{1}}\left(\boldsymbol{p}^{(m+1)}, t\right) \exp \left[i \boldsymbol{k}_{1} \cdot \boldsymbol{q}_{1}^{(m+1)}\right], \\
& R_{f}=\sum_{\boldsymbol{k}_{0} \neq \mathbf{0}} R_{\boldsymbol{k}_{0} \boldsymbol{k}_{1}}\left(\boldsymbol{p}^{(m+1)}, t\right) \exp \left[i \boldsymbol{k}_{0} \cdot \boldsymbol{q}_{0}^{(m+1)}+i \boldsymbol{k}_{1} \cdot \boldsymbol{q}_{1}^{(m+1)}\right] .
\end{aligned}
$$

Since $R_{s}$ is written as

$$
R_{s}=\frac{1}{(2 \pi)^{n_{0}}} \int_{0}^{2 \pi} \cdots \int_{0}^{2 \pi} d^{n_{0}} \boldsymbol{q}_{0}^{(m+1)} R
$$

$R_{s}$ is bounded as

$$
\left|t^{m+1} R_{s}\right| \leq\left|t^{m+1} R\right| \leq \epsilon^{m} M .
$$

Then

$$
\left|t^{m+1} R_{f}\right| \leq\left|t^{m+1}\left(R-R_{s}\right)\right| \leq 2 \epsilon^{m} M .
$$

We define

$$
A_{m+1}\left(\boldsymbol{q}_{1}^{(m+1)}, \boldsymbol{p}^{(m+1)}, t\right)=A_{m}\left(\boldsymbol{q}_{1}^{(m+1)}, \boldsymbol{p}^{(m+1)}, t\right)+R_{s}\left(\boldsymbol{q}_{1}^{(m+1)}, \boldsymbol{p}^{(m+1)}, t\right),
$$

and

$$
B_{m+1}\left(\boldsymbol{q}^{(m+1)}, \boldsymbol{p}^{(m+1)}, t\right)=R_{f}\left(\boldsymbol{q}^{(m+1)}, \boldsymbol{p}^{(m+1)}, t\right) .
$$

We evaluate

$$
\begin{aligned}
& \left|t A_{m+1}\right| \leq M_{1}^{(m+1)}, \\
& \left|t^{m+1}\left\{A_{m+1}\left(\boldsymbol{q}_{1}^{(m+1)}, \boldsymbol{p}^{(m+1)}, t\right)-A_{m}\left(\boldsymbol{q}_{1}^{(m+1)}, \boldsymbol{p}^{(m+1)}, t\right)\right\}\right| \leq \epsilon^{m} \frac{M_{2}^{(m+1)}}{2}, \\
& \left|t^{m+1} B_{m+1}\right| \leq \epsilon^{m} M_{2}^{(m+1)},
\end{aligned}
$$


where

$$
\begin{aligned}
& M_{1}^{(m+1)}=M_{1}^{(m)}+\left(1+\rho_{m+1}\right)^{m} \epsilon^{m} M, \\
& M_{2}^{(m+1)}=2 M,
\end{aligned}
$$

on the domain $D\left(\rho_{m+1}\right)$. We complete the proof.

\section{$\S B \quad$ Appendix; Proof of Propositions 4.2A, 4.2B}

\section{B.1 Proof of the Proposition 4.2A}

We consider the system obtained by three times of canonical transformations.

$$
H^{(1)} \stackrel{S_{3}}{\rightarrow} H^{(2)} \stackrel{S_{2}}{\rightarrow} H^{(3)} \stackrel{S_{3}}{\rightarrow} H^{(4)} .
$$

We evaluate in how good accuracy the truncated system $H_{t r}^{(4)}$ approximates $H^{(4)}$.

$$
\begin{aligned}
H_{t r}^{(4)} & =\frac{2}{\epsilon}(\boldsymbol{\omega} \cdot \boldsymbol{P})^{1 / 2}+A_{4}\left(\boldsymbol{Q}_{1}, \boldsymbol{P}, t\right) \\
H^{(4)} & =\frac{2}{\epsilon}(\boldsymbol{\omega} \cdot \boldsymbol{p})^{1 / 2}+A_{4}\left(\boldsymbol{q}_{1}, \boldsymbol{p}, t\right)+B_{4}(\boldsymbol{q}, \boldsymbol{p}, t),
\end{aligned}
$$

where

$$
\left|t A_{4}\right| \leq M_{1}^{(4)}, \quad\left|t^{4} B_{4}\right| \leq \epsilon^{3} M_{2}^{(4)}
$$

By taking the difference between

$$
\frac{d \boldsymbol{p}_{0}}{d t}=-\frac{\partial B_{4}}{\partial \boldsymbol{q}_{0}}
$$

and

$$
\frac{d \boldsymbol{P}_{0}}{d t}=0
$$

we obtain

$$
\frac{d}{d t} \Delta \boldsymbol{P}_{0}=-\frac{\partial B_{4}}{\partial \boldsymbol{q}_{0}}
$$

By integrating the above inequality, we obtain

$$
\begin{aligned}
\left|\Delta \boldsymbol{P}_{0}(t)\right| & \leq\left|\Delta \boldsymbol{P}_{0}(1)\right|+\int_{1} d t \frac{\epsilon^{3}}{t^{4}} \\
& \leq\left|\Delta \boldsymbol{P}_{0}(1)\right|+\epsilon^{3} .
\end{aligned}
$$

If we fix

$$
\left|\Delta \boldsymbol{P}_{0}(1)\right| \leq \epsilon^{3}
$$

we obtain

$$
\left|\Delta \boldsymbol{P}_{0}\right| \leq \epsilon^{3}
$$


Next we evalutate $\Delta \boldsymbol{Q}_{1}=\boldsymbol{q}_{1}-\boldsymbol{Q}_{1}$. By taking the difference between

$$
\frac{d \boldsymbol{q}_{1}}{d t}=\frac{\boldsymbol{\omega}_{1}}{\epsilon} \frac{1}{(\boldsymbol{\omega} \cdot \boldsymbol{p})^{1 / 2}}+\frac{\partial A_{4}}{\partial \boldsymbol{p}_{1}}+\frac{\partial B_{4}}{\partial \boldsymbol{p}_{1}},
$$

and

$$
\frac{d \boldsymbol{Q}_{1}}{d t}=\frac{\boldsymbol{\omega}_{1}}{\epsilon} \frac{1}{(\boldsymbol{\omega} \cdot \boldsymbol{P})^{1 / 2}}+\frac{\partial A_{4}}{\partial \boldsymbol{P}_{1}}
$$

we obtain

$$
\frac{d}{d t} \Delta \boldsymbol{Q}_{1}=\frac{\boldsymbol{\omega}_{1}}{\epsilon}\left[\frac{1}{(\boldsymbol{\omega} \cdot \boldsymbol{p})^{1 / 2}}-\frac{1}{(\boldsymbol{\omega} \cdot \boldsymbol{P})^{1 / 2}}\right]+\left[\frac{\partial A_{4}}{\partial \boldsymbol{p}_{1}}-\frac{\partial A_{4}}{\partial \boldsymbol{P}_{1}}\right]+\frac{\partial B_{4}}{\partial \boldsymbol{p}_{1}} .
$$

By using the mean value theorem,

$$
\begin{aligned}
& \frac{1}{(\boldsymbol{\omega} \cdot \boldsymbol{p})^{1 / 2}}-\frac{1}{(\boldsymbol{\omega} \cdot \boldsymbol{P})^{1 / 2}}=-\frac{1}{2} \frac{1}{(\boldsymbol{\omega} \cdot \boldsymbol{P})^{3 / 2}}\left(\boldsymbol{\omega}_{0} \cdot \Delta \boldsymbol{P}_{0}+\boldsymbol{\omega}_{1} \cdot \Delta \boldsymbol{P}_{1}\right), \\
& \frac{\partial A_{4}}{\partial \boldsymbol{p}_{1}}-\frac{\partial A_{4}}{\partial \boldsymbol{P}_{1}}=\left[\Delta \boldsymbol{P}_{0} \cdot \frac{\partial}{\partial \boldsymbol{P}_{0}}+\Delta \boldsymbol{Q}_{1} \cdot \frac{\partial}{\partial \boldsymbol{Q}_{1}}+\Delta \boldsymbol{P}_{1} \cdot \frac{\partial}{\partial \boldsymbol{P}_{1}}\right] \frac{\partial A_{4}}{\partial \boldsymbol{P}_{1}},
\end{aligned}
$$

are obtained where the differentiations in the right hand side are taken at appropriate values between $\left(\boldsymbol{p}_{0}, \boldsymbol{z}\right)$ and $\left(\boldsymbol{P}_{0}, \boldsymbol{Z}\right)$. Since we are only interested in the upper bounds of the coefficients, we understand that the differentiations are taken at appropriate values between the original variables $\left(\boldsymbol{p}_{0}, \boldsymbol{z}\right)$ and the truncated variables $\left(\boldsymbol{P}_{0}, \boldsymbol{Z}\right)$ without notice from now on. Then we get

$$
\begin{aligned}
\frac{d}{d t} \Delta \boldsymbol{Q}_{1}= & -\frac{1}{2} \frac{\boldsymbol{\omega}_{1}}{\epsilon} \frac{1}{(\boldsymbol{\omega} \cdot \boldsymbol{P})^{3 / 2}}\left(\boldsymbol{\omega}_{0} \cdot \Delta \boldsymbol{P}_{0}+\boldsymbol{\omega}_{1} \cdot \Delta \boldsymbol{P}_{1}\right) \\
& +\left[\Delta \boldsymbol{P}_{0} \cdot \frac{\partial}{\partial \boldsymbol{P}_{0}}+\Delta \boldsymbol{Q}_{1} \cdot \frac{\partial}{\partial \boldsymbol{Q}_{1}}+\Delta \boldsymbol{P}_{1} \cdot \frac{\partial}{\partial \boldsymbol{P}_{1}}\right] \frac{\partial A_{4}}{\partial \boldsymbol{P}_{1}}+\frac{\partial B_{4}}{\partial \boldsymbol{p}_{1}} .
\end{aligned}
$$

In the same way, as for $\Delta \boldsymbol{P}_{1}=\boldsymbol{p}_{1}-\boldsymbol{P}_{1}$, we obtain

$$
\frac{d}{d t} \Delta \boldsymbol{P}_{1}=-\left[\Delta \boldsymbol{P}_{0} \cdot \frac{\partial}{\partial \boldsymbol{P}_{0}}+\Delta \boldsymbol{Q}_{1} \cdot \frac{\partial}{\partial \boldsymbol{Q}_{1}}+\Delta \boldsymbol{P}_{1} \cdot \frac{\partial}{\partial \boldsymbol{P}_{1}}\right] \frac{\partial A_{4}}{\partial \boldsymbol{Q}_{1}}-\frac{\partial B_{4}}{\partial \boldsymbol{q}_{1}} .
$$

By using the notation $\boldsymbol{z}=\left(\boldsymbol{q}_{1}, \boldsymbol{p}_{1}\right), \boldsymbol{Z}=\left(\boldsymbol{Q}_{1}, \boldsymbol{P}_{1}\right), \Delta \boldsymbol{Z}=\boldsymbol{z}-\boldsymbol{Z}$, we obtain

$$
\frac{d}{d t}|\Delta \boldsymbol{Z}| \leq\left(\frac{\boldsymbol{\omega}_{1}^{2}}{\epsilon}+\frac{\Gamma}{t}\right)|\Delta \boldsymbol{Z}|+\left(\frac{\boldsymbol{\omega}_{1}}{\epsilon}+\frac{1}{t}\right)\left|\Delta \boldsymbol{P}_{0}\right|+\frac{\epsilon^{3}}{t^{4}},
$$

Under the assumption $\boldsymbol{\omega}_{1} \sim \epsilon$, we get

$$
\frac{d}{d t}|\Delta \boldsymbol{Z}| \leq\left(\frac{\Gamma}{t}+\epsilon\right)|\Delta \boldsymbol{Z}|+\left|\Delta \boldsymbol{P}_{0}\right|+\frac{\epsilon^{3}}{t^{4}} .
$$

By integrating the above inequality, we obtain

$$
|\Delta \boldsymbol{Z}| \leq \exp \left[\int_{1} d t\left(\frac{\Gamma}{t}+\epsilon\right)\right]\left[|\Delta \boldsymbol{Z}(1)|+\int_{1} d t\left|\Delta \boldsymbol{P}_{0}\right|+\int_{1} d t \frac{\epsilon^{3}}{t^{4}}\right] .
$$


By using (B.10), and assuming

$$
|\Delta \boldsymbol{Z}(1)| \leq \epsilon^{3}
$$

we obtain

$$
|\Delta \boldsymbol{Z}| \leq t^{\Gamma} \exp [\epsilon(t-1)]\left[\epsilon^{3} t+\epsilon^{3}\right] .
$$

We consider the time interval as

$$
1 \leq t \leq \frac{1}{t^{\beta}}
$$

where

$$
\beta=\frac{1}{\Gamma+1}<1 .
$$

In this interval, we obtain the evaluation as

$$
|\Delta Z| \leq \epsilon^{2}
$$

The equation for $\Delta \boldsymbol{Q}_{0}=\boldsymbol{q}_{0}-\boldsymbol{Q}_{0}$ is given by

$$
\begin{aligned}
\frac{d}{d t} \Delta \boldsymbol{Q}_{0}= & -\frac{1}{2} \frac{\boldsymbol{\omega}_{0}}{\epsilon} \frac{1}{(\boldsymbol{\omega} \cdot \boldsymbol{P})^{3 / 2}}\left(\boldsymbol{\omega}_{0} \cdot \Delta \boldsymbol{P}_{0}+\boldsymbol{\omega}_{1} \cdot \Delta \boldsymbol{P}_{1}\right) \\
& +\left[\Delta \boldsymbol{P}_{0} \cdot \frac{\partial}{\partial \boldsymbol{P}_{0}}+\Delta \boldsymbol{Q}_{1} \cdot \frac{\partial}{\partial \boldsymbol{Q}_{1}}+\Delta \boldsymbol{P}_{1} \cdot \frac{\partial}{\partial \boldsymbol{P}_{1}}\right] \frac{\partial A_{4}}{\partial \boldsymbol{P}_{0}}+\frac{\partial B_{4}}{\partial \boldsymbol{p}_{0}},
\end{aligned}
$$

which yields

$$
\left|\frac{d}{d t} \Delta \boldsymbol{Q}_{0}\right| \leq\left(\frac{1}{\epsilon}+\frac{1}{t}\right)\left|\Delta \boldsymbol{P}_{0}\right|+\frac{1}{t}\left|\Delta \boldsymbol{Q}_{1}\right|+\left(1+\frac{1}{t}\right)\left|\Delta \boldsymbol{P}_{1}\right|+\frac{\epsilon^{3}}{t^{4}} .
$$

By integrating the above inequality, we obtain

$$
\left|\Delta \boldsymbol{Q}_{0}\right| \leq\left|\Delta \boldsymbol{Q}_{0}(1)\right|+\frac{1}{\epsilon} \int_{1} d t\left|\Delta \boldsymbol{P}_{0}\right|+\int_{1} \frac{d t}{t}\left|\Delta \boldsymbol{Q}_{1}\right|+\int_{1} d t\left|\Delta \boldsymbol{P}_{1}\right|+\int_{1} d t \frac{\epsilon^{3}}{t^{4}},
$$

which yields

$$
\begin{aligned}
\left|\frac{\Delta \boldsymbol{Q}_{0}}{t}\right| & \leq\left|\Delta \boldsymbol{Q}_{0}(1)\right|+\frac{1}{\epsilon}\left|\Delta \boldsymbol{P}_{0}\right|+|\Delta \boldsymbol{Z}|+\epsilon^{3} \\
& \leq \epsilon^{2},
\end{aligned}
$$

where we used (B.10), (B.25) and assumed that

$$
\left|\Delta \boldsymbol{Q}_{0}(1)\right| \leq \epsilon^{2} .
$$

By obtaining (B.10), (B.25), (B.29), we complete the proof of (4.59).

Next we consider in how good accuracy the perturbations of the truncated system $H_{t r}^{(4)}$ approximate the perturbations of the transformed system $H^{(4)}$. We try to obtain the equation for $\Delta \delta \boldsymbol{Q}_{0}=\delta \boldsymbol{q}_{0}-\delta \boldsymbol{Q}_{0}$. We take the difference between

$$
\begin{aligned}
\frac{d}{d t} \delta \boldsymbol{q}_{0}= & -\frac{1}{2} \frac{\boldsymbol{\omega}_{0}}{\epsilon} \frac{1}{(\boldsymbol{\omega} \cdot \boldsymbol{p})^{3 / 2}}\left(\boldsymbol{\omega}_{0} \cdot \delta \boldsymbol{p}_{0}+\boldsymbol{\omega}_{1} \cdot \delta \boldsymbol{p}_{1}\right) \\
& +\left(\delta \boldsymbol{p}_{0} \cdot \frac{\partial}{\partial \boldsymbol{p}_{0}}+\delta \boldsymbol{q}_{1} \cdot \frac{\partial}{\partial \boldsymbol{q}_{1}}+\delta \boldsymbol{p}_{1} \cdot \frac{\partial}{\partial \boldsymbol{p}_{1}}\right) \frac{\partial A_{4}}{\partial \boldsymbol{p}_{0}} \\
& +\left(\delta \boldsymbol{q}_{0} \cdot \frac{\partial}{\partial \boldsymbol{q}_{0}}+\delta \boldsymbol{p}_{0} \cdot \frac{\partial}{\partial \boldsymbol{p}_{0}}+\delta \boldsymbol{q}_{1} \cdot \frac{\partial}{\partial \boldsymbol{q}_{1}}+\delta \boldsymbol{p}_{1} \cdot \frac{\partial}{\partial \boldsymbol{p}_{1}}\right) \frac{\partial B_{4}}{\partial \boldsymbol{p}_{0}}
\end{aligned}
$$


and

$$
\frac{d}{d t} \delta \boldsymbol{Q}_{0}=-\frac{1}{2} \frac{\boldsymbol{\omega}_{0}}{\epsilon} \frac{1}{(\boldsymbol{\omega} \cdot \boldsymbol{P})^{3 / 2}}\left(\boldsymbol{\omega}_{0} \cdot \delta \boldsymbol{P}_{0}+\boldsymbol{\omega}_{1} \cdot \delta \boldsymbol{P}_{1}\right)+\left(\delta \boldsymbol{P}_{0} \cdot \frac{\partial}{\partial \boldsymbol{P}_{0}}+\delta \boldsymbol{Q}_{1} \cdot \frac{\partial}{\partial \boldsymbol{Q}_{1}}+\delta \boldsymbol{P}_{1} \cdot \frac{\partial}{\partial \boldsymbol{P}_{1}}\right) \frac{\partial A_{4}}{\partial \boldsymbol{P}_{0}} .
$$

By using the mean value theorem, we obtain

$$
\begin{aligned}
&- \frac{1}{2} \frac{\boldsymbol{\omega}_{0}}{\epsilon} \frac{1}{(\boldsymbol{\omega} \cdot \boldsymbol{p})^{3 / 2}}\left(\boldsymbol{\omega}_{0} \cdot \delta \boldsymbol{p}_{0}+\boldsymbol{\omega}_{1} \cdot \delta \boldsymbol{p}_{1}\right)+\frac{1}{2} \frac{\boldsymbol{\omega}_{0}}{\epsilon} \frac{1}{(\boldsymbol{\omega} \cdot \boldsymbol{P})^{3 / 2}}\left(\boldsymbol{\omega}_{0} \cdot \delta \boldsymbol{P}_{0}+\boldsymbol{\omega}_{1} \cdot \delta \boldsymbol{P}_{1}\right) \\
&=- \frac{1}{2} \frac{\boldsymbol{\omega}_{0}}{\epsilon} \frac{1}{(\boldsymbol{\omega} \cdot \boldsymbol{p})^{3 / 2}}\left(\boldsymbol{\omega}_{0} \cdot \Delta \delta \boldsymbol{P}_{0}+\boldsymbol{\omega}_{1} \cdot \Delta \delta \boldsymbol{P}_{1}\right) \\
&+\frac{3}{4} \frac{\boldsymbol{\omega}_{0}}{\epsilon} \frac{1}{(\boldsymbol{\omega} \cdot \boldsymbol{P})^{5 / 2}}\left(\boldsymbol{\omega}_{0} \cdot \Delta \boldsymbol{P}_{0}+\boldsymbol{\omega}_{1} \cdot \Delta \boldsymbol{P}_{1}\right)\left(\boldsymbol{\omega}_{0} \cdot \delta \boldsymbol{P}_{0}+\boldsymbol{\omega}_{1} \cdot \delta \boldsymbol{P}_{1}\right), \\
&\left(\delta \boldsymbol{p}_{0} \cdot \frac{\partial}{\partial \boldsymbol{p}_{0}}+\delta \boldsymbol{q}_{1} \cdot \frac{\partial}{\partial \boldsymbol{q}_{1}}+\delta \boldsymbol{p}_{1} \cdot \frac{\partial}{\partial \boldsymbol{p}_{1}}\right) \frac{\partial A_{4}}{\partial \boldsymbol{p}_{0}}-\left(\delta \boldsymbol{P}_{0} \cdot \frac{\partial}{\partial \boldsymbol{P}_{0}}+\delta \boldsymbol{Q}_{1} \cdot \frac{\partial}{\partial \boldsymbol{Q}_{1}}+\delta \boldsymbol{P}_{1} \cdot \frac{\partial}{\partial \boldsymbol{P}_{1}}\right) \frac{\partial A_{4}}{\partial \boldsymbol{P}_{0}} \\
&=\left(\Delta \delta \boldsymbol{P}_{0} \cdot \frac{\partial}{\partial \boldsymbol{p}_{0}}+\Delta \delta \boldsymbol{Q}_{1} \cdot \frac{\partial}{\partial \boldsymbol{q}_{1}}+\Delta \delta \boldsymbol{P}_{1} \cdot \frac{\partial}{\partial \boldsymbol{p}_{1}}\right) \frac{\partial A_{4}}{\partial \boldsymbol{p}_{0}} \\
&+\left(\Delta \boldsymbol{P}_{0} \cdot \frac{\partial}{\partial \boldsymbol{P}_{0}}+\Delta \boldsymbol{Q}_{1} \cdot \frac{\partial}{\partial \boldsymbol{Q}_{1}}+\Delta \boldsymbol{P}_{1} \cdot \frac{\partial}{\partial \boldsymbol{P}_{1}}\right) \\
&\left(\delta \boldsymbol{P}_{0} \cdot \frac{\partial}{\partial \boldsymbol{P}_{0}}+\delta \boldsymbol{Q}_{1} \cdot \frac{\partial}{\partial \boldsymbol{Q}_{1}}+\delta \boldsymbol{P}_{1} \cdot \frac{\partial}{\partial \boldsymbol{P}_{1}}\right) \frac{\partial A_{4}}{\partial \boldsymbol{P}_{0}},
\end{aligned}
$$

and

$$
\begin{aligned}
& \left(\delta \boldsymbol{q}_{0} \cdot \frac{\partial}{\partial \boldsymbol{q}_{0}}+\delta \boldsymbol{p}_{0} \cdot \frac{\partial}{\partial \boldsymbol{p}_{0}}+\delta \boldsymbol{q}_{1} \cdot \frac{\partial}{\partial \boldsymbol{q}_{1}}+\delta \boldsymbol{p}_{1} \cdot \frac{\partial}{\partial \boldsymbol{p}_{1}}\right) \frac{\partial B_{4}}{\partial \boldsymbol{p}_{0}} \\
& =\left(\delta \boldsymbol{Q}_{0} \cdot \frac{\partial}{\partial \boldsymbol{q}_{0}}+\delta \boldsymbol{P}_{0} \cdot \frac{\partial}{\partial \boldsymbol{p}_{0}}+\delta \boldsymbol{Q}_{1} \cdot \frac{\partial}{\partial \boldsymbol{q}_{1}}+\delta \boldsymbol{P}_{1} \cdot \frac{\partial}{\partial \boldsymbol{p}_{1}}\right) \frac{\partial B_{4}}{\partial \boldsymbol{p}_{0}} \\
& \quad+\left(\Delta \delta \boldsymbol{Q}_{0} \cdot \frac{\partial}{\partial \boldsymbol{q}_{0}}+\Delta \delta \boldsymbol{P}_{0} \cdot \frac{\partial}{\partial \boldsymbol{p}_{0}}+\Delta \delta \boldsymbol{Q}_{1} \cdot \frac{\partial}{\partial \boldsymbol{q}_{1}}+\Delta \delta \boldsymbol{P}_{1} \cdot \frac{\partial}{\partial \boldsymbol{p}_{1}}\right) \frac{\partial B_{4}}{\partial \boldsymbol{p}_{0}},
\end{aligned}
$$

where the differentiations in the right hand side are taken at the appropriate values between $(\boldsymbol{Q}, \boldsymbol{P})$ and $(\boldsymbol{q}, \boldsymbol{p})$, and it will not be noticed from now on. By evaluating the coefficients, we obtain

$$
\begin{aligned}
\left|\frac{d}{d t} \Delta \delta \boldsymbol{Q}_{0}\right| \leq & \frac{1}{\epsilon}\left(\left|\Delta \delta \boldsymbol{P}_{0}\right|+\boldsymbol{\omega}_{1}\left|\Delta \delta \boldsymbol{P}_{1}\right|\right)+\frac{1}{\epsilon}\left(\left|\Delta \boldsymbol{P}_{0}\right|+\omega_{1}\left|\Delta \boldsymbol{P}_{1}\right|\right)\left(\left|\delta \boldsymbol{P}_{0}\right|+\omega_{1}\left|\delta \boldsymbol{P}_{1}\right|\right) \\
& +\left(\left|\Delta \delta \boldsymbol{P}_{0}\right|+\left|\Delta \delta \boldsymbol{Q}_{1}\right|+\left|\Delta \delta \boldsymbol{P}_{1}\right|\right) \frac{1}{t} \\
& +\left(\left|\Delta \boldsymbol{P}_{0}\right|+\left|\Delta \boldsymbol{Q}_{1}\right|+\left|\Delta \boldsymbol{P}_{1}\right|\right)\left(\left|\delta \boldsymbol{P}_{0}\right|+\left|\delta \boldsymbol{Q}_{1}\right|+\left|\delta \boldsymbol{P}_{1}\right|\right) \frac{1}{t} \\
& +\left(\left|\delta \boldsymbol{Q}_{0}\right|+\left|\delta \boldsymbol{P}_{0}\right|+\left|\delta \boldsymbol{Q}_{1}\right|+\left|\delta \boldsymbol{P}_{1}\right|\right) \frac{\epsilon^{3}}{t^{4}} \\
& +\left(\left|\Delta \delta \boldsymbol{Q}_{0}\right|+\left|\Delta \delta \boldsymbol{P}_{0}\right|+\left|\Delta \delta \boldsymbol{Q}_{1}\right|+\left|\Delta \delta \boldsymbol{P}_{1}\right|\right) \frac{\epsilon^{3}}{t^{4}}
\end{aligned}
$$


By using the inequalities as

$$
\begin{aligned}
& \left|\Delta \boldsymbol{P}_{0}\right|+\left|\Delta \boldsymbol{Q}_{1}\right|+\left|\Delta \boldsymbol{P}_{1}\right| \leq \epsilon^{2}, \\
& \left|\Delta \boldsymbol{P}_{0}\right|+\boldsymbol{\omega}_{1}\left|\Delta \boldsymbol{P}_{1}\right| \leq \epsilon^{3},
\end{aligned}
$$

we obtain

$$
\begin{aligned}
\left|\frac{d}{d t} \Delta \delta \boldsymbol{Q}_{0}\right| \leq & \left(\frac{1}{\epsilon}+\frac{1}{t}\right)\left|\Delta \delta \boldsymbol{P}_{0}\right|+\left|\Delta \delta \boldsymbol{P}_{1}\right|+\frac{1}{t}\left|\Delta \delta \boldsymbol{Q}_{1}\right|+\frac{\epsilon^{3}}{t^{4}}\left|\Delta \delta \boldsymbol{Q}_{0}\right| \\
& +\epsilon^{2}\left|\delta \boldsymbol{P}_{0}\right|+\epsilon^{2}\left(\epsilon+\frac{1}{t}\right)\left|\delta \boldsymbol{P}_{1}\right|+\frac{\epsilon^{2}}{t}\left|\delta \boldsymbol{Q}_{1}\right|+\frac{\epsilon^{3}}{t^{4}}\left|\delta \boldsymbol{Q}_{0}\right| .
\end{aligned}
$$

In the same way as $\Delta \delta \boldsymbol{Q}_{0}$, we obtain

$$
\begin{aligned}
\left|\frac{d}{d t} \Delta \delta \boldsymbol{P}_{0}\right| \leq & \left(\left|\Delta \delta \boldsymbol{Q}_{0}\right|+\left|\Delta \delta \boldsymbol{P}_{0}\right|+\left|\Delta \delta \boldsymbol{Q}_{1}\right|+\left|\Delta \delta \boldsymbol{P}_{1}\right|\right) \frac{\epsilon^{3}}{t^{4}} \\
& +\left(\left|\delta \boldsymbol{Q}_{0}\right|+\left|\delta \boldsymbol{P}_{0}\right|+\left|\delta \boldsymbol{Q}_{1}\right|+\left|\delta \boldsymbol{P}_{1}\right|\right) \frac{\epsilon^{3}}{t^{4}}
\end{aligned}
$$

and

$$
\begin{aligned}
& \frac{d}{d t}|\Delta \delta \boldsymbol{Z}| \leq\left(\frac{\Gamma}{t}+\epsilon\right)|\Delta \delta \boldsymbol{Z}|+\left|\Delta \delta \boldsymbol{P}_{0}\right|+\frac{\epsilon^{3}}{t^{4}}\left|\Delta \delta \boldsymbol{Q}_{0}\right| \\
& +\epsilon^{2}\left(\epsilon+\frac{1}{t}\right)\left|\delta \boldsymbol{P}_{0}\right|+\frac{\epsilon^{2}}{t}\left|\delta \boldsymbol{Q}_{1}\right|+\epsilon^{2}\left(\epsilon^{2}+\frac{1}{t}\right)\left|\delta \boldsymbol{P}_{1}\right|+\frac{\epsilon^{3}}{t^{4}}\left|\delta \boldsymbol{Q}_{0}\right| .
\end{aligned}
$$

By using

$$
\left|\Delta \delta \boldsymbol{Q}_{1}\right|,\left|\Delta \delta \boldsymbol{P}_{1}\right| \leq|\Delta \delta \boldsymbol{Z}|, \quad\left|\delta \boldsymbol{Q}_{1}\right|,\left|\delta \boldsymbol{P}_{1}\right| \leq|\delta \boldsymbol{Z}|
$$

we obtain

$$
\begin{aligned}
\frac{d}{d t}|\Delta \delta \boldsymbol{Z}| \leq & \left(\epsilon+\frac{\Gamma}{t}\right)|\Delta \delta \boldsymbol{Z}|+\left|\Delta \delta \boldsymbol{P}_{0}\right|+\frac{\epsilon^{3}}{t^{4}}\left|\Delta \delta \boldsymbol{Q}_{0}\right| \\
+ & \epsilon^{2}\left|\delta \boldsymbol{P}_{0}\right|+\epsilon^{2}|\delta \boldsymbol{Z}|+\frac{\epsilon^{3}}{t^{4}}\left|\delta \boldsymbol{Q}_{0}\right|, \\
\left|\frac{d}{d t} \Delta \delta \boldsymbol{Q}_{0}\right| \leq & \frac{1}{\epsilon}\left|\Delta \delta \boldsymbol{P}_{0}\right|+|\Delta \delta \boldsymbol{Z}|+\frac{\epsilon^{3}}{t^{4}}\left|\Delta \delta \boldsymbol{Q}_{0}\right| \\
& +\epsilon^{2}\left|\delta \boldsymbol{P}_{0}\right|+\epsilon^{2}|\delta \boldsymbol{Z}|+\frac{\epsilon^{3}}{t^{4}}\left|\delta \boldsymbol{Q}_{0}\right|,
\end{aligned}
$$

and

$$
\begin{aligned}
\left|\frac{d}{d t} \Delta \delta \boldsymbol{P}_{0}\right| \leq & \left(\left|\Delta \delta \boldsymbol{Q}_{0}\right|+\left|\Delta \delta \boldsymbol{P}_{0}\right|+|\Delta \delta \boldsymbol{Z}|\right) \frac{\epsilon^{3}}{t^{4}} \\
& +\left(\left|\delta \boldsymbol{Q}_{0}\right|+\left|\delta \boldsymbol{P}_{0}\right|+|\delta \boldsymbol{Z}|\right) \frac{\epsilon^{3}}{t^{4}}
\end{aligned}
$$


The inequality (B.43) yields

$$
\begin{aligned}
|\Delta \delta \boldsymbol{Z}| \leq & \exp \left[\int_{1} d t\left(\epsilon+\frac{\Gamma}{t}\right)\right] \\
& \left\{|\Delta \delta \boldsymbol{Z}(1)|+\int_{1} d t\left|\Delta \delta \boldsymbol{P}_{0}\right|+\int_{1} d t \frac{\epsilon^{3}}{t^{4}}\left|\Delta \delta \boldsymbol{Q}_{0}\right|\right. \\
& \left.+\int_{1} d t \epsilon^{2}\left|\delta \boldsymbol{P}_{0}\right|+\int_{1} d t \epsilon^{2}|\delta \boldsymbol{Z}|+\int_{1} d t \frac{\epsilon^{3}}{t^{4}}\left|\delta \boldsymbol{Q}_{0}\right|\right\} \\
\leq & t^{\Gamma} \exp [\epsilon(t-1)] \\
& \left\{|\Delta \delta \boldsymbol{Z}(1)|+t\left\|\Delta \delta \boldsymbol{P}_{0}\right\|+\epsilon^{3}\left\|\frac{\Delta \delta \boldsymbol{Q}_{0}}{t^{2}}\right\|+\epsilon^{2} t\left\|\delta \boldsymbol{P}_{0}\right\|\right. \\
& \left.+\epsilon^{2} t\|\delta \boldsymbol{Z}\|+\epsilon^{3}\left\|\frac{\delta \boldsymbol{Q}_{0}}{t^{2}}\right\|\right\} .
\end{aligned}
$$

The inequality (B.44) yields

$$
\begin{aligned}
\left|\Delta \delta \boldsymbol{Q}_{0}\right| \leq & \exp \left[\int_{1} d t \frac{\epsilon^{3}}{t^{4}}\right] \\
& \left\{\left|\Delta \delta \boldsymbol{Q}_{0}(1)\right|+\frac{t}{\epsilon}\left\|\Delta \delta \boldsymbol{P}_{0}\right\|+t\|\Delta \delta \boldsymbol{Z}\|\right. \\
& \left.+\epsilon^{2} t\left\|\delta \boldsymbol{P}_{0}\right\|+\epsilon^{2} t\|\delta \boldsymbol{Z}\|+\epsilon^{3}\left\|\frac{\delta \boldsymbol{Q}_{0}}{t^{2}}\right\|\right\} \\
\leq & \left\{\left|\Delta \delta \boldsymbol{Q}_{0}(1)\right|+\frac{t}{\epsilon}\left\|\Delta \delta \boldsymbol{P}_{0}\right\|+t\|\Delta \delta \boldsymbol{Z}\|\right. \\
& \left.+\epsilon^{2} t\left\|\delta \boldsymbol{P}_{0}\right\|+\epsilon^{2} t\|\delta \boldsymbol{Z}\|+\epsilon^{3}\left\|\frac{\delta \boldsymbol{Q}_{0}}{t^{2}}\right\|\right\},
\end{aligned}
$$

and the inequality (B.45) yields

$$
\begin{aligned}
\left|\Delta \delta \boldsymbol{P}_{0}\right| \leq & \left|\Delta \delta \boldsymbol{P}_{0}(1)\right| \\
& +\epsilon^{3}\left(\left\|\frac{\Delta \delta \boldsymbol{Q}_{0}}{t^{2}}\right\|+\left\|\frac{\Delta \delta \boldsymbol{Z}}{t^{2}}\right\|+\left\|\frac{\delta \boldsymbol{Q}_{0}}{t^{2}}\right\|+\left\|\frac{\delta \boldsymbol{P}_{0}}{t^{2}}\right\|+\left\|\frac{\delta \boldsymbol{Z}}{t^{2}}\right\|\right) .
\end{aligned}
$$

If we consider the time interval

$$
1 \leq t \leq \frac{1}{t^{\beta}}
$$

where

$$
\beta=\frac{1}{\Gamma+1} \leq 1,
$$

the growth factor of $|\Delta \delta \boldsymbol{Z}|$ is bounded as

$$
t^{\Gamma} \exp [\epsilon(t-1)] t \leq \frac{1}{\epsilon} .
$$


Then in this interval (B.49), we obtain

$$
\begin{aligned}
\left|\frac{\Delta \delta \boldsymbol{Q}_{0}}{t}\right| \leq & \left|\Delta \delta \boldsymbol{Q}_{0}(1)\right|+\frac{1}{\epsilon}\left\|\Delta \delta \boldsymbol{P}_{0}\right\|+\|\Delta \delta \boldsymbol{Z}\| \\
& +\epsilon^{2}\left\|\delta \boldsymbol{P}_{0}\right\|+\epsilon^{2}\|\delta \boldsymbol{Z}\|+\epsilon^{3}\left\|\frac{\delta \boldsymbol{Q}_{0}}{t^{2}}\right\|, \\
\left|\Delta \delta \boldsymbol{P}_{0}\right| \leq & \left|\Delta \delta \boldsymbol{P}_{0}(1)\right|+\epsilon^{3}\left(\left\|\frac{\Delta \delta \boldsymbol{Q}_{0}}{t^{2}}\right\|+\left\|\frac{\Delta \delta \boldsymbol{Z}}{t^{2}}\right\|\right. \\
& \left.+\left\|\frac{\delta \boldsymbol{Q}_{0}}{t^{2}}\right\|+\left\|\frac{\delta \boldsymbol{P}_{0}}{t^{2}}\right\|+\left\|\frac{\delta \boldsymbol{Z}}{t^{2}}\right\|\right), \\
|\Delta \delta \boldsymbol{Z}| \leq & \frac{1}{\epsilon}|\Delta \delta \boldsymbol{Z}(1)|+\frac{1}{\epsilon}\left\|\Delta \delta \boldsymbol{P}_{0}\right\|+\epsilon^{2}\left\|\frac{\Delta \delta \boldsymbol{Q}_{0}}{t^{2}}\right\| \\
& +\epsilon\left\|\delta \boldsymbol{P}_{0}\right\|+\epsilon\|\delta \boldsymbol{Z}\|+\epsilon^{2}\left\|\frac{\delta \boldsymbol{Q}_{0}}{t^{2}}\right\|,
\end{aligned}
$$

By using (B.53) to (B.52) (B.54), we obtain

$$
\begin{aligned}
\left\|\frac{\Delta \delta \boldsymbol{Q}_{0}}{t}\right\| \leq & \left|\Delta \delta \boldsymbol{Q}_{0}(1)\right|+\frac{1}{\epsilon}\left|\Delta \delta \boldsymbol{P}_{0}(1)\right|+\|\Delta \delta \boldsymbol{Z}\| \\
& +\epsilon^{2}\left\|\frac{\delta \boldsymbol{Q}_{0}}{t^{2}}\right\|,+\epsilon^{2}\|\delta \boldsymbol{Z}\|+\epsilon^{2}\left\|\delta \boldsymbol{P}_{0}\right\|,
\end{aligned}
$$

and

$$
\begin{aligned}
\|\Delta \delta \boldsymbol{Z}\| \leq & \frac{1}{\epsilon}|\Delta \delta \boldsymbol{Z}(1)|+\frac{1}{\epsilon}\left|\Delta \delta \boldsymbol{P}_{0}(1)\right| \\
& +\epsilon^{2}\left\|\frac{\Delta \delta \boldsymbol{Q}_{0}}{t^{2}}\right\|+\epsilon^{2}\left\|\frac{\delta \boldsymbol{Q}_{0}}{t^{2}}\right\|+\epsilon\left\|\delta \boldsymbol{P}_{0}\right\|+\epsilon\|\delta \boldsymbol{Z}\| .
\end{aligned}
$$

By using (B.56) to (B.55), we get

$$
\begin{aligned}
\left\|\frac{\Delta \delta \boldsymbol{Q}_{0}}{t}\right\| \leq & \left|\Delta \delta \boldsymbol{Q}_{0}(1)\right|+\frac{1}{\epsilon}\left|\Delta \delta \boldsymbol{P}_{0}(1)\right|+\frac{1}{\epsilon}|\Delta \delta \boldsymbol{Z}(1)| \\
& +\epsilon^{2}\left\|\frac{\delta \boldsymbol{Q}_{0}}{t^{2}}\right\|,+\epsilon\left\|\delta \boldsymbol{P}_{0}\right\|+\epsilon\|\delta \boldsymbol{Z}\|,
\end{aligned}
$$

By using (B.57) to (B.56), we obtain

$$
\begin{aligned}
\|\Delta \delta \boldsymbol{Z}\| \leq & \epsilon^{2}\left|\Delta \delta \boldsymbol{Q}_{0}(1)\right|+\frac{1}{\epsilon}\left|\Delta \delta \boldsymbol{P}_{0}(1)\right|+\frac{1}{\epsilon}|\Delta \delta \boldsymbol{Z}(1)| \\
& +\epsilon^{2}\left\|\frac{\delta \boldsymbol{Q}_{0}}{t^{2}}\right\|+\epsilon\left\|\delta \boldsymbol{P}_{0}\right\|+\epsilon\|\delta \boldsymbol{Z}\| .
\end{aligned}
$$


By using (B.57) (B.58) to (B.53), we obtain

$$
\begin{aligned}
\left\|\Delta \delta \boldsymbol{P}_{0}\right\| \leq & \epsilon^{3}\left|\Delta \delta \boldsymbol{Q}_{0}(1)\right|+\left|\Delta \delta \boldsymbol{P}_{0}(1)\right|+\epsilon^{2}|\Delta \delta \boldsymbol{Z}(1)| \\
& +\epsilon^{3}\left\|\frac{\delta \boldsymbol{Q}_{0}}{t^{2}}\right\|+\epsilon^{3}\left\|\delta \boldsymbol{P}_{0}\right\|+\epsilon^{3}\|\delta \boldsymbol{Z}\| .
\end{aligned}
$$

By substituting

$$
\Delta \delta \boldsymbol{Q}_{0}(1)=\Delta \delta \boldsymbol{P}_{0}(1)=\Delta \delta \boldsymbol{Z}(1)=0
$$

to (B.57) (B.58) (B.59), we obtain

$$
\begin{aligned}
\left\|\frac{\Delta \delta \boldsymbol{Q}_{0}}{t}\right\| & \leq \epsilon^{2}\left\|\frac{\delta \boldsymbol{Q}_{0}}{t^{2}}\right\|+\epsilon\left\|\delta \boldsymbol{P}_{0}\right\|+\epsilon\|\delta \boldsymbol{Z}\|, \\
\left\|\Delta \delta \boldsymbol{P}_{0}\right\| & \leq \epsilon^{3}\left\|\frac{\delta \boldsymbol{Q}_{0}}{t^{2}}\right\|+\epsilon^{3}\left\|\delta \boldsymbol{P}_{0}\right\|+\epsilon^{3}\|\delta \boldsymbol{Z}\|, \\
\|\Delta \delta \boldsymbol{Z}\| & \leq \epsilon^{2}\left\|\frac{\delta \boldsymbol{Q}_{0}}{t^{2}}\right\|+\epsilon\left\|\delta \boldsymbol{P}_{0}\right\|+\epsilon\|\delta \boldsymbol{Z}\| .
\end{aligned}
$$

Next we evaluate $\left\|\delta \boldsymbol{Q}_{0} / t^{2}\right\|$ by

$$
\begin{aligned}
\frac{d}{d t} \delta \boldsymbol{Q}_{0}= & -\frac{1}{2} \frac{\boldsymbol{\omega}_{0}}{\epsilon} \frac{1}{(\boldsymbol{\omega} \cdot \boldsymbol{P})^{3 / 2}}\left(\boldsymbol{\omega}_{0} \cdot \delta \boldsymbol{P}_{0}+\boldsymbol{\omega}_{1} \cdot \delta \boldsymbol{P}_{1}\right) \\
& +\left(\delta \boldsymbol{P}_{0} \cdot \frac{\partial}{\partial \boldsymbol{P}_{0}}+\delta \boldsymbol{Q}_{1} \cdot \frac{\partial}{\partial \boldsymbol{Q}_{1}}+\delta \boldsymbol{P}_{1} \cdot \frac{\partial}{\partial \boldsymbol{P}_{1}}\right) \frac{\partial A_{4}}{\partial \boldsymbol{P}_{0}}
\end{aligned}
$$

which yields

$$
\left\|\frac{\delta \boldsymbol{Q}_{0}}{t}\right\| \leq\left|\delta \boldsymbol{Q}_{0}(1)\right|+\frac{1}{\epsilon}\left\|\delta \boldsymbol{P}_{0}\right\|+\|\delta \boldsymbol{Z}\| .
$$

By using (B.65) to (B.61) (B.62) (B.63), we get

$$
\begin{aligned}
\left\|\frac{\Delta \delta \boldsymbol{Q}_{0}}{t}\right\| & \leq \epsilon^{2}\left|\delta \boldsymbol{Q}_{0}(1)\right|+\epsilon\left\|\delta \boldsymbol{P}_{0}\right\|+\epsilon\|\delta \boldsymbol{Z}\|, \\
\left\|\Delta \delta \boldsymbol{P}_{0}\right\| & \leq \epsilon^{3}\left|\delta \boldsymbol{Q}_{0}(1)\right|+\epsilon^{2}\left\|\delta \boldsymbol{P}_{0}\right\|+\epsilon^{3}\|\delta \boldsymbol{Z}\|, \\
\|\Delta \delta \boldsymbol{Z}\| & \leq \epsilon^{2}\left|\delta \boldsymbol{Q}_{0}(1)\right|+\epsilon\left\|\delta \boldsymbol{P}_{0}\right\|+\epsilon\|\delta \boldsymbol{Z}\| .
\end{aligned}
$$

These complete the proof of (4.60).

\section{B.2 Proof of Proposition 4.2B}

We evaluate how the errors $\Delta \boldsymbol{Q}^{(4)}, \Delta \boldsymbol{P}^{(4)}, \Delta \delta \boldsymbol{Q}^{(4)}, \Delta \delta \boldsymbol{P}^{(4)}$ are transmitted to $\Delta \boldsymbol{Q}^{(1)}$, $\Delta \boldsymbol{P}^{(1)}, \Delta \delta \boldsymbol{Q}^{(1)}, \Delta \delta \boldsymbol{P}^{(1)}$ by the canonical transformations

$$
\begin{aligned}
\boldsymbol{p}^{(m)} & =\boldsymbol{p}^{(m+1)}+\frac{\partial S_{m}}{\partial \boldsymbol{q}^{(m)}}, \\
\boldsymbol{q}^{(m+1)} & =\boldsymbol{q}^{(m)}+\frac{\partial S_{m}}{\partial \boldsymbol{p}^{(m+1)}}, \quad(m \geq 1)
\end{aligned}
$$


where

$$
\left|t^{m} S_{m}\right| \leq \epsilon^{m} L_{1}^{(m)}
$$

Lemma

$$
\begin{aligned}
\left|\Delta \boldsymbol{P}^{(1)}-\Delta \boldsymbol{P}^{(m)}\right| & \leq \frac{\epsilon}{t}\left|\Delta \boldsymbol{Q}^{(m)}\right|+\frac{\epsilon}{t}\left|\Delta \boldsymbol{P}^{(m)}\right|, \\
\left|\Delta \boldsymbol{Q}^{(1)}-\Delta \boldsymbol{Q}^{(m)}\right| & \leq \frac{\epsilon}{t}\left|\Delta \boldsymbol{Q}^{(m)}\right|+\frac{\epsilon}{t}\left|\Delta \boldsymbol{P}^{(m)}\right|, \\
\left|\delta \boldsymbol{P}^{(1)}-\delta \boldsymbol{P}^{(m)}\right| & \leq \frac{\epsilon}{t}\left|\delta \boldsymbol{Q}^{(m)}\right|+\frac{\epsilon}{t}\left|\delta \boldsymbol{P}^{(m)}\right|, \\
\left|\delta \boldsymbol{Q}^{(1)}-\delta \boldsymbol{Q}^{(m)}\right| & \leq \frac{\epsilon}{t}\left|\delta \boldsymbol{Q}^{(m)}\right|+\frac{\epsilon}{t}\left|\delta \boldsymbol{P}^{(m)}\right|,
\end{aligned}
$$

Proof of Lemma

We can prove (B.72), (B.73) in the almost same way. So we prove (B.72) as the representative. We consider the difference $\Delta \boldsymbol{P}=\boldsymbol{p}-\boldsymbol{P}$. Taking the difference between

$$
\boldsymbol{p}^{(m)}=\boldsymbol{p}^{(m+1)}+\frac{\partial S_{m}}{\partial \boldsymbol{q}^{(m)}},
$$

and

$$
\boldsymbol{P}^{(m)}=\boldsymbol{P}^{(m+1)}+\frac{\partial S_{m}}{\partial \boldsymbol{Q}^{(m)}}
$$

yields

$$
\begin{aligned}
\Delta \boldsymbol{P}^{(m)} & =\Delta \boldsymbol{P}^{(m+1)}+\frac{\partial S_{m}}{\partial \boldsymbol{q}^{(m)}}-\frac{\partial S_{m}}{\partial \boldsymbol{Q}^{(m)}} \\
& =\Delta \boldsymbol{P}^{(m+1)}+\left(\Delta \boldsymbol{Q}^{(m)} \cdot \frac{\partial}{\partial \boldsymbol{Q}^{(m)}}+\Delta \boldsymbol{P}^{(m+1)} \cdot \frac{\partial}{\partial \boldsymbol{P}^{(m+1)}}\right) \frac{\partial S_{m}}{\partial \boldsymbol{Q}^{(m)}}
\end{aligned}
$$

where differentiations in the right hand side are taken at the appropriate values between $(\boldsymbol{q}, \boldsymbol{p})$ and $(\boldsymbol{Q}, \boldsymbol{P})$ according the mean value theorem. So we obtain

$$
\begin{aligned}
& \left|\Delta \boldsymbol{P}^{(m)}-\Delta \boldsymbol{P}^{(m+1)}\right| \leq \frac{\epsilon^{m}}{t^{m}}\left(\left|\Delta \boldsymbol{Q}^{(m)}\right|+\left|\Delta \boldsymbol{P}^{(m+1)}\right|\right), \\
& \left|\Delta \boldsymbol{Q}^{(m)}-\Delta \boldsymbol{Q}^{(m+1)}\right| \leq \frac{\epsilon^{m}}{t^{m}}\left(\left|\Delta \boldsymbol{Q}^{(m)}\right|+\left|\Delta \boldsymbol{P}^{(m+1)}\right|\right),
\end{aligned}
$$

As for the first term in the right hand side of the above inequalities, we obtain

$$
\begin{aligned}
\left|\Delta \boldsymbol{Q}^{(m)}\right| & \leq\left|\Delta \boldsymbol{Q}^{(m)}-\Delta \boldsymbol{Q}^{(m+1)}\right|+\left|\Delta \boldsymbol{Q}^{(m+1)}\right| \\
& \leq \frac{\epsilon^{m}}{t^{m}}\left(\left|\Delta \boldsymbol{Q}^{(m)}\right|+\left|\Delta \boldsymbol{P}^{(m+1)}\right|\right)+\left|\Delta \boldsymbol{Q}^{(m+1)}\right|
\end{aligned}
$$

which yields

$$
\left|\Delta \boldsymbol{Q}^{(m)}\right| \leq\left|\Delta \boldsymbol{Q}^{(m+1)}\right|+\frac{\epsilon^{m}}{t^{m}}\left|\Delta \boldsymbol{P}^{(m+1)}\right|
$$


By using (B.79) to (B.77), we obtain

$$
\begin{aligned}
& \left|\Delta \boldsymbol{P}^{(m)}-\Delta \boldsymbol{P}^{(m+1)}\right| \leq \frac{\epsilon^{m}}{t^{m}}\left|\Delta \boldsymbol{X}^{(m+1)}\right|, \\
& \left|\Delta \boldsymbol{Q}^{(m)}-\Delta \boldsymbol{Q}^{(m+1)}\right| \leq \frac{\epsilon^{m}}{t^{m}}\left|\Delta \boldsymbol{X}^{(m+1)}\right|,
\end{aligned}
$$

where

$$
\left|\Delta \boldsymbol{X}^{(m)}\right|=\left|\Delta \boldsymbol{Q}^{(m)}\right|+\left|\Delta \boldsymbol{P}^{(m)}\right| .
$$

In the same way as (B.79), we obtain

$$
\left|\Delta \boldsymbol{P}^{(m)}\right|=\left|\Delta \boldsymbol{P}^{(m+1)}\right|+\frac{\epsilon^{m}}{t^{m}}\left|\Delta \boldsymbol{Q}^{(m+1)}\right| .
$$

From (B.79) (B.82), we obtain

$$
\left|\Delta \boldsymbol{X}^{(m)}\right| \leq\left|\Delta \boldsymbol{X}^{(m+1)}\right|
$$

We evaluate

$$
\begin{aligned}
\left|\Delta \boldsymbol{P}^{(1)}-\Delta \boldsymbol{P}^{(m)}\right| & \leq\left|\Delta \boldsymbol{P}^{(1)}-\Delta \boldsymbol{P}^{(2)}\right|+\left|\Delta \boldsymbol{P}^{(2)}-\Delta \boldsymbol{P}^{(3)}\right|+\cdots+\left|\Delta \boldsymbol{P}^{(m-1)}-\Delta \boldsymbol{P}^{(m)}\right| \\
& \leq \frac{\epsilon}{t}\left|\Delta \boldsymbol{X}^{(2)}\right|+\frac{\epsilon^{2}}{t^{2}}\left|\Delta \boldsymbol{X}^{(3)}\right|+\cdots+\frac{\epsilon^{m-1}}{t^{m-1}}\left|\Delta \boldsymbol{X}^{(m)}\right| \\
& \leq \frac{\epsilon}{t}\left|\Delta \boldsymbol{X}^{(m)}\right|
\end{aligned}
$$

where we used (B.80) (B.83). In the same way, we get

$$
\left|\Delta \boldsymbol{Q}^{(1)}-\Delta \boldsymbol{Q}^{(m)}\right| \leq \frac{\epsilon}{t}\left|\Delta \boldsymbol{X}^{(m)}\right| .
$$

These complete the proof.

Proof of Lemma

From the inequalities (4.59), we obtain

$$
\frac{1}{t}\left|\Delta \boldsymbol{Q}^{(4)}\right|+\frac{1}{t}\left|\Delta \boldsymbol{P}^{(4)}\right| \leq \epsilon^{2}
$$

So

$$
\begin{aligned}
\left|\Delta \boldsymbol{P}^{(1)}-\Delta \boldsymbol{P}^{(4)}\right| & \leq \epsilon^{3} \\
\left|\Delta \boldsymbol{Q}^{(1)}-\Delta \boldsymbol{Q}^{(4)}\right| & \leq \epsilon^{3}
\end{aligned}
$$

are obtained. Therefore we obtain

$$
\begin{aligned}
\left|\Delta \boldsymbol{P}_{0}^{(1)}\right| & \leq\left|\Delta \boldsymbol{P}_{0}^{(4)}\right|+\left|\Delta \boldsymbol{P}_{0}^{(1)}-\Delta \boldsymbol{P}_{0}^{(4)}\right| \leq \epsilon^{3}, \\
\left|\Delta \boldsymbol{Z}^{(1)}\right| & \leq\left|\Delta \boldsymbol{Z}^{(4)}\right|+\left|\Delta \boldsymbol{Z}^{(1)}-\Delta \boldsymbol{Z}^{(4)}\right| \leq \epsilon^{2}, \\
\left|\frac{\Delta \boldsymbol{Q}_{0}^{(1)}}{t}\right| & \leq\left|\frac{\Delta \boldsymbol{Q}_{0}^{(4)}}{t}\right|+\left|\frac{\Delta \boldsymbol{Q}_{0}^{(1)}}{t}-\frac{\Delta \boldsymbol{Q}_{0}^{(4)}}{t}\right| \leq \epsilon^{2},
\end{aligned}
$$


We have proved (4.63).

Lemma

$$
\begin{aligned}
\left|\Delta \delta \boldsymbol{P}^{(1)}-\Delta \delta \boldsymbol{P}^{(m)}\right| & \leq \frac{\epsilon}{t}\left(\left|\delta \boldsymbol{X}^{(m)}\right|\left|\Delta \boldsymbol{X}^{(m)}\right|+\left|\Delta \delta \boldsymbol{X}^{(m)}\right|\right), \\
\left|\Delta \delta \boldsymbol{Q}^{(1)}-\Delta \delta \boldsymbol{Q}^{(m)}\right| & \leq \frac{\epsilon}{t}\left(\left|\delta \boldsymbol{X}^{(m)}\right|\left|\Delta \boldsymbol{X}^{(m)}\right|+\left|\Delta \delta \boldsymbol{X}^{(m)}\right|\right),
\end{aligned}
$$

where

$$
\left|\Delta \delta \boldsymbol{X}^{(m)}\right|=\left|\Delta \delta \boldsymbol{Q}^{(m)}\right|+\left|\Delta \delta \boldsymbol{P}^{(m)}\right|
$$

Proof of Lemma

By using (B.79), we obtain

$$
\left|\Delta \boldsymbol{Q}^{(m)}\right|+\left|\Delta \boldsymbol{P}^{(m+1)}\right| \leq\left|\Delta \boldsymbol{X}^{(m+1)}\right|
$$

In the same way,

$$
\left|\delta \boldsymbol{Q}^{(m)}\right|+\left|\delta \boldsymbol{P}^{(m+1)}\right| \leq\left|\delta \boldsymbol{X}^{(m+1)}\right|
$$

is obtained. We take the difference between

$$
\delta \boldsymbol{p}^{(m)}=\delta \boldsymbol{p}^{(m+1)}+\left(\delta \boldsymbol{q}^{(m)} \cdot \frac{\partial}{\partial \boldsymbol{q}^{(m)}}+\delta \boldsymbol{p}^{(m+1)} \cdot \frac{\partial}{\partial \boldsymbol{p}^{(m+1)}}\right) \frac{\partial S_{m}}{\partial \boldsymbol{q}^{(m)}},
$$

and

$$
\delta \boldsymbol{P}^{(m)}=\delta \boldsymbol{P}^{(m+1)}+\left(\delta \boldsymbol{Q}^{(m)} \cdot \frac{\partial}{\partial \boldsymbol{Q}^{(m)}}+\delta \boldsymbol{P}^{(m+1)} \cdot \frac{\partial}{\partial \boldsymbol{P}^{(m+1)}}\right) \frac{\partial S_{m}}{\partial \boldsymbol{Q}^{(m)}} .
$$

By the mean value theorem, we get

$$
\begin{aligned}
& \left(\delta \boldsymbol{q}^{(m)} \cdot \frac{\partial}{\partial \boldsymbol{q}^{(m)}}+\delta \boldsymbol{p}^{(m+1)} \cdot \frac{\partial}{\partial \boldsymbol{p}^{(m+1)}}\right) \frac{\partial S_{m}}{\partial \boldsymbol{q}^{(m)}} \\
& -\left(\delta \boldsymbol{Q}^{(m)} \cdot \frac{\partial}{\partial \boldsymbol{Q}^{(m)}}+\delta \boldsymbol{P}^{(m+1)} \cdot \frac{\partial}{\partial \boldsymbol{P}^{(m+1)}}\right) \frac{\partial S_{m}}{\partial \boldsymbol{Q}^{(m)}} \\
= & \left(\delta \boldsymbol{Q}^{(m)} \cdot \frac{\partial}{\partial \boldsymbol{Q}^{(m)}}+\delta \boldsymbol{P}^{(m+1)} \cdot \frac{\partial}{\partial \boldsymbol{P}^{(m+1)}}\right)\left(\Delta \boldsymbol{Q}^{(m)} \cdot \frac{\partial}{\partial \boldsymbol{Q}^{(m)}}+\Delta \boldsymbol{P}^{(m+1)} \cdot \frac{\partial}{\partial \boldsymbol{P}^{(m+1)}}\right) \frac{\partial S_{m}}{\partial \boldsymbol{Q}^{(m)}} \\
+ & \left(\Delta \delta \boldsymbol{Q}^{(m)} \cdot \frac{\partial}{\partial \boldsymbol{q}^{(m)}}+\Delta \delta \boldsymbol{P}^{(m+1)} \cdot \frac{\partial}{\partial \boldsymbol{p}^{(m+1)}}\right) \frac{\partial S_{m}}{\partial \boldsymbol{q}^{(m)}},
\end{aligned}
$$

where the differentiations in the right hand side are taken at the appropriate values between $(\boldsymbol{q}, \boldsymbol{p})$ and $(\boldsymbol{Q}, \boldsymbol{P})$. So we obtain

$$
\begin{aligned}
\left|\Delta \delta \boldsymbol{P}^{(m)}-\Delta \delta \boldsymbol{P}^{(m+1)}\right| \leq & \left(\left|\delta \boldsymbol{Q}^{(m)}\right|+\left|\delta \boldsymbol{P}^{(m+1)}\right|\right)\left(\left|\Delta \boldsymbol{Q}^{(m)}\right|+\left|\Delta \boldsymbol{P}^{(m+1)}\right|\right) \frac{\epsilon^{m}}{t^{m}} \\
& +\left(\left|\Delta \delta \boldsymbol{Q}^{(m)}\right|+\left|\Delta \delta \boldsymbol{P}^{(m+1)}\right|\right) \frac{\epsilon^{m}}{t^{m}} \\
\leq & \frac{\epsilon^{m}}{t^{m}}\left|\delta \boldsymbol{X}^{(m+1)}\right|\left|\Delta \boldsymbol{X}^{(m+1)}\right| \\
& +\frac{\epsilon^{m}}{t^{m}}\left(\left|\Delta \delta \boldsymbol{Q}^{(m)}\right|+\left|\Delta \delta \boldsymbol{P}^{(m+1)}\right|\right),
\end{aligned}
$$


where (B.93) (B.94) are used. In the same way, we get

$$
\begin{aligned}
\left|\Delta \delta \boldsymbol{Q}^{(m)}-\Delta \delta \boldsymbol{Q}^{(m+1)}\right| \leq & \frac{\epsilon^{m}}{t^{m}}\left|\delta \boldsymbol{X}^{(m+1)}\right|\left|\Delta \boldsymbol{X}^{(m+1)}\right| \\
& +\frac{\epsilon^{m}}{t^{m}}\left(\left|\Delta \delta \boldsymbol{Q}^{(m)}\right|+\left|\Delta \delta \boldsymbol{P}^{(m+1)}\right|\right) .
\end{aligned}
$$

From the above inequality,

$$
\begin{aligned}
\left|\Delta \delta \boldsymbol{Q}^{(m)}\right| \leq & \left|\Delta \delta \boldsymbol{Q}^{(m)}-\Delta \delta \boldsymbol{Q}^{(m+1)}\right|+\left|\Delta \delta \boldsymbol{Q}^{(m)}\right| \\
\leq & \frac{\epsilon^{m}}{t^{m}}\left|\delta \boldsymbol{X}^{(m+1)}\right|\left|\Delta \boldsymbol{X}^{(m+1)}\right|+\frac{\epsilon^{m}}{t^{m}}\left(\left|\Delta \delta \boldsymbol{Q}^{(m)}\right|+\left|\Delta \delta \boldsymbol{P}^{(m+1)}\right|\right) \\
& +\left|\Delta \delta \boldsymbol{Q}^{(m+1)}\right|
\end{aligned}
$$

which yields

$$
\left|\Delta \delta \boldsymbol{Q}^{(m)}\right| \leq \frac{\epsilon^{m}}{t^{m}}\left|\delta \boldsymbol{X}^{(m+1)}\right|\left|\Delta \boldsymbol{X}^{(m+1)}\right|+\left|\Delta \delta \boldsymbol{Q}^{(m+1)}\right|+\frac{\epsilon^{m}}{t^{m}}\left|\Delta \delta \boldsymbol{P}^{(m+1)}\right| .
$$

Then we get

$$
\left|\Delta \delta \boldsymbol{Q}^{(m)}\right|+\left|\Delta \delta \boldsymbol{P}^{(m+1)}\right| \leq \frac{\epsilon^{m}}{t^{m}}\left|\delta \boldsymbol{X}^{(m+1)}\right|\left|\Delta \boldsymbol{X}^{(m+1)}\right|+\left|\Delta \delta \boldsymbol{X}^{(m+1)}\right| .
$$

By using (B.102) to (B.98) (B.99), we obtain

$$
\begin{aligned}
\left|\Delta \delta \boldsymbol{P}^{(m)}-\Delta \delta \boldsymbol{P}^{(m+1)}\right| & \leq \frac{\epsilon^{m}}{t^{m}}\left(\left|\delta \boldsymbol{X}^{(m+1)}\right|\left|\Delta \boldsymbol{X}^{(m+1)}\right|+\left|\Delta \delta \boldsymbol{X}^{(m+1)}\right|\right) \\
\left|\Delta \delta \boldsymbol{Q}^{(m)}-\Delta \delta \boldsymbol{Q}^{(m+1)}\right| & \leq \frac{\epsilon^{m}}{t^{m}}\left(\left|\delta \boldsymbol{X}^{(m+1)}\right|\left|\Delta \boldsymbol{X}^{(m+1)}\right|+\left|\Delta \delta \boldsymbol{X}^{(m+1)}\right|\right)
\end{aligned}
$$

By using the above inequalities,

$$
\begin{aligned}
\left|\Delta \delta \boldsymbol{X}^{(m)}\right| \leq & \left|\Delta \delta \boldsymbol{X}^{(m+1)}\right| \\
& +\frac{\epsilon^{m}}{t^{m}}\left(\left|\delta \boldsymbol{X}^{(m+1)}\right|\left|\Delta \boldsymbol{X}^{(m+1)}\right|+\left|\Delta \delta \boldsymbol{X}^{(m+1)}\right|\right),
\end{aligned}
$$

is obtained. Then we get

$$
\begin{aligned}
\left|\Delta \delta \boldsymbol{P}^{(1)}-\Delta \delta \boldsymbol{P}^{(m)}\right| & \leq\left|\Delta \delta \boldsymbol{P}^{(1)}-\Delta \delta \boldsymbol{P}^{(2)}\right|+\cdots+\left|\Delta \delta \boldsymbol{P}^{(m-1)}-\Delta \delta \boldsymbol{P}^{(m)}\right| \\
& \leq \frac{\epsilon}{t}\left(\left|\delta \boldsymbol{X}^{(m)}\right|\left|\Delta \boldsymbol{X}^{(m)}\right|+\left|\Delta \delta \boldsymbol{X}^{(m)}\right|\right) .
\end{aligned}
$$

In the same way, we obtain

$$
\left|\Delta \delta \boldsymbol{Q}^{(1)}-\Delta \delta \boldsymbol{Q}^{(m)}\right| \leq \frac{\epsilon}{t}\left(\left|\delta \boldsymbol{X}^{(m)}\right|\left|\Delta \boldsymbol{X}^{(m)}\right|+\left|\Delta \delta \boldsymbol{X}^{(m)}\right|\right) .
$$

These complete the proof.

Proof End of Lemma

From the inequalities (4.59), we obtain

$$
\left|\frac{\Delta \boldsymbol{X}^{(4)}}{t}\right| \leq \epsilon^{2} .
$$


From the inequalities (B.65), we obtain

$$
\left|\delta \boldsymbol{X}^{(4)}\right| \leq\left|\delta \boldsymbol{Q}_{0}(1)\right| t+\frac{1}{\epsilon}\left\|\delta \boldsymbol{P}_{0}\right\| t+\|\delta \boldsymbol{Z}\| t .
$$

From the inequalities (4.60), we obtain

$$
\frac{1}{t}\left|\Delta \delta \boldsymbol{X}^{(4)}\right| \leq \epsilon^{2}\left|\delta \boldsymbol{Q}_{0}^{(4)}(1)\right|+\epsilon\left\|\delta \boldsymbol{P}_{0}^{(4)}\right\|+\epsilon\left\|\delta \boldsymbol{Z}^{(4)}\right\|
$$

By using the above three inequlities, we obtain

$$
\begin{aligned}
&\left|\Delta \delta \boldsymbol{P}^{(1)}-\Delta \delta \boldsymbol{P}^{(4)}\right| \leq \epsilon^{2}\left|\delta \boldsymbol{Q}_{0}^{(4)}(1)\right|+\epsilon\left\|\delta \boldsymbol{P}_{0}^{(4)}\right\|+\epsilon^{2}\left\|\delta \boldsymbol{Z}^{(4)}\right\| \\
&\left|\Delta \delta \boldsymbol{Q}^{(1)}-\Delta \delta \boldsymbol{Q}^{(4)}\right| \leq \epsilon^{2}\left|\delta \boldsymbol{Q}_{0}^{(4)}(1)\right|+\epsilon\left\|\delta \boldsymbol{P}_{0}^{(4)}\right\|+\epsilon^{2}\left\|\delta \boldsymbol{Z}^{(4)}\right\|,
\end{aligned}
$$

for $1 \leq t \leq 1 / \epsilon$. By combining (4.60) (B.110) (B.111), we obtain

$$
\begin{aligned}
\left\|\frac{\Delta \delta \boldsymbol{Q}_{0}^{(1)}}{t}\right\| & \leq \epsilon^{2}\left|\delta \boldsymbol{Q}_{0}^{(4)}(1)\right|+\epsilon\left\|\delta \boldsymbol{P}_{0}^{(4)}\right\|+\epsilon\left\|\delta \boldsymbol{Z}^{(4)}\right\|, \\
\left\|\Delta \delta \boldsymbol{P}_{0}^{(1)}\right\| & \leq \epsilon^{2}\left|\delta \boldsymbol{Q}_{0}^{(4)}(1)\right|+\epsilon\left\|\delta \boldsymbol{P}_{0}^{(4)}\right\|+\epsilon^{2}\left\|\delta \boldsymbol{Z}^{(4)}\right\|, \\
\left\|\Delta \delta \boldsymbol{Z}^{(1)}\right\| & \leq \epsilon^{2}\left|\delta \boldsymbol{Q}_{0}^{(4)}(1)\right|+\epsilon\left\|\delta \boldsymbol{P}_{0}^{(4)}\right\|+\epsilon\left\|\delta \boldsymbol{Z}^{(4)}\right\| .
\end{aligned}
$$

This completes the proof of the propositionB.

\section{$\S \mathrm{C} \quad$ Appendix; Proof of Proposition 5.3}

The proof is essentially the same as that of the preceding proposition 4.1. So in this proof we estimate the generating function of the canonical transformation, and the residual parts which cannot be eliminated by the canonical transformation only roughly. Later we omit $m$. We consider the canonical transformation induced by the generating function given by

$$
T_{l}\left(\boldsymbol{q}_{1}^{(l)}, \boldsymbol{p}^{(l+1)}, t\right)=\sum_{\boldsymbol{k}_{1} \neq \mathbf{0}} T_{\boldsymbol{k}_{1}}\left(\boldsymbol{p}^{(l+1)}, t\right) e^{i \boldsymbol{k}_{1} \cdot \boldsymbol{q}_{1}^{(l)}}
$$

where $T_{l}$ does not depend on $\boldsymbol{q}_{0}^{(l)}$.

The transformed Hamiltonian is

$$
\begin{aligned}
H^{(l+1)} & =H^{(l)}+\frac{\partial T_{l}}{\partial t} \\
& =\frac{2}{\epsilon^{2}}\left(\boldsymbol{\omega} \cdot \boldsymbol{p}^{(l+1)}\right)^{1 / 2}+\frac{1}{\epsilon^{2}} \frac{1}{\left(\boldsymbol{\omega} \cdot \boldsymbol{p}^{(l+1)}\right)^{1 / 2}} \boldsymbol{\omega}_{1} \cdot \frac{\partial T_{l}\left(\boldsymbol{q}_{1}^{(l)}, \boldsymbol{p}^{(l+1)}, t\right)}{\partial \boldsymbol{q}_{1}^{(l)}}+R_{1} \\
& +D_{l}\left(\boldsymbol{p}^{(l+1)}, t\right)+R_{2}+E_{l}\left(\boldsymbol{q}_{1}^{(l)}, \boldsymbol{p}^{(l+1)}, t\right)+R_{3} \\
& +B_{l}\left(\boldsymbol{q}^{(l+1)}, \boldsymbol{p}^{(l+1)}, t\right)+R_{4}+\frac{\partial T_{l}\left(\boldsymbol{q}_{1}^{(l)}, \boldsymbol{p}^{(l+1)}, t\right)}{\partial t}
\end{aligned}
$$


where $R_{1}, \cdots R_{4}$ are defined as

$$
\begin{aligned}
& R_{1}=\frac{2}{\epsilon^{2}}\left(\boldsymbol{\omega} \cdot \boldsymbol{p}^{(l)}\right)^{1 / 2}-\frac{2}{\epsilon^{2}}\left(\boldsymbol{\omega} \cdot \boldsymbol{p}^{(l+1)}\right)^{1 / 2}-\frac{1}{\epsilon^{2}} \frac{\boldsymbol{\omega}_{1}}{\left(\boldsymbol{\omega} \cdot \boldsymbol{p}^{(l+1)}\right)^{1 / 2}} \cdot\left(\boldsymbol{p}_{1}^{(l)}-\boldsymbol{p}_{1}^{(l+1)}\right), \\
& R_{2}=D_{l}\left(\boldsymbol{p}^{(l)}, t\right)-D_{l}\left(\boldsymbol{p}^{(l+1)}, t\right) \\
& R_{3}=E_{l}\left(\boldsymbol{q}_{1}^{(l)}, \boldsymbol{p}^{(l)}, t\right)-E_{l}\left(\boldsymbol{q}_{1}^{(l)}, \boldsymbol{p}^{(l+1)}, t\right) \\
& R_{4}=B_{l}\left(\boldsymbol{q}^{(l)}, \boldsymbol{p}^{(l)}, t\right)-B_{l}\left(\boldsymbol{q}^{(l+1)}, \boldsymbol{p}^{(l+1)}, t\right) .
\end{aligned}
$$

We determine the generating function $T_{l}$ so that the leading term depending on the slow angle variables $\boldsymbol{q}_{1}^{(l)}$ can be eliminated;

$$
\frac{1}{\epsilon^{2}} \frac{1}{\left(\boldsymbol{\omega} \cdot \boldsymbol{p}^{(l+1)}\right)^{1 / 2}} \boldsymbol{\omega}_{1} \cdot \frac{\partial T_{l}\left(\boldsymbol{q}_{1}^{(l)}, \boldsymbol{p}^{(l+1)}, t\right)}{\partial \boldsymbol{q}_{1}^{(l)}}=-E_{l}\left(\boldsymbol{q}_{1}^{(l)}, \boldsymbol{p}^{(l+1)}, t\right) .
$$

When $E_{l}$ is decomposed as

$$
E_{l}=\sum_{\boldsymbol{k}_{1} \neq \mathbf{0}} e_{\boldsymbol{k}_{1}}\left(\boldsymbol{p}^{(l+1)}, t\right) e^{i \boldsymbol{k}_{1} \cdot \boldsymbol{q}_{1}^{(l)}}
$$

$T_{l}$ is written as

$$
T_{l}=\sum_{\boldsymbol{k}_{1} \neq \mathbf{0}} i \epsilon^{2}\left(\boldsymbol{\omega} \cdot \boldsymbol{p}^{(l+1)}\right)^{1 / 2} \frac{1}{\left(\boldsymbol{\omega}_{1} \cdot \boldsymbol{k}_{1}\right)} e_{\boldsymbol{k}_{1}} e^{i \boldsymbol{k}_{1} \cdot \boldsymbol{q}_{1}^{(l)}} .
$$

Since $T_{l}$ is roughly estimated as

$$
T_{l} \sim \frac{\epsilon^{l}}{t^{l}},
$$

the differences between the original variables and the transformed variables are estimated as

$$
\begin{aligned}
& \boldsymbol{p}_{0}^{(l)}-\boldsymbol{p}_{0}^{(l+1)}=\mathbf{0}, \\
& \boldsymbol{p}_{1}^{(l)}-\boldsymbol{p}_{1}^{(l+1)} \sim \frac{\epsilon^{l}}{t^{l}}, \\
& \boldsymbol{q}^{(l+1)}-\boldsymbol{q}^{(l)} \sim \frac{\epsilon^{l}}{t^{l}} .
\end{aligned}
$$

By using these estimates, we evaluate the residual parts $R_{i}$ and $\partial T_{l} / \partial t$;

$$
\left|R_{1}\right| \leq\left[-\frac{1}{4} \frac{1}{\epsilon^{2}} \frac{\boldsymbol{\omega}_{1}^{i} \boldsymbol{\omega}_{1}^{j}}{(\boldsymbol{\omega} \cdot \boldsymbol{p})^{3 / 2}}\left(\boldsymbol{p}_{1}^{(l)}-\boldsymbol{p}_{1}^{(l+1)}\right)^{i}\left(\boldsymbol{p}_{1}^{(l)}-\boldsymbol{p}_{1}^{(l+1)}\right)^{j}\right] \sim \frac{\epsilon^{2 l}}{t^{2 l}},
$$

where we use $\boldsymbol{\omega}_{1} \sim \epsilon$,

$$
\begin{aligned}
\left|R_{2}\right| & \leq\left[\frac{\partial D_{l}(\boldsymbol{p}, t)}{\partial \boldsymbol{p}_{1}} \cdot\left(\boldsymbol{p}_{1}^{(l)}-\boldsymbol{p}_{1}^{(l+1)}\right)\right] \sim \frac{\epsilon^{l+1}}{t^{l+2}} \\
\left|R_{3}\right| & \leq\left[\frac{\partial E_{l}}{\partial \boldsymbol{p}_{1}} \cdot\left(\boldsymbol{p}_{1}^{(l)}-\boldsymbol{p}_{1}^{(l+1)}\right)\right] \sim \frac{\epsilon^{2 l-1}}{t^{2 l}} \\
\left|R_{4}\right| & \leq\left[\frac{\partial B_{l}}{\partial \boldsymbol{q}} \cdot\left(\boldsymbol{q}^{(l)}-\boldsymbol{q}^{(l+1)}\right)+\frac{\partial B_{l}}{\partial \boldsymbol{p}_{1}} \cdot\left(\boldsymbol{p}_{1}^{(l)}-\boldsymbol{p}_{1}^{(l+1)}\right)\right] \sim \frac{\epsilon^{2(m-1)}}{t^{m}} \frac{\epsilon^{l}}{t^{l}} \\
\frac{\partial T_{l}}{\partial t} & \sim \frac{\epsilon^{l}}{t^{l+1}} .
\end{aligned}
$$


Then $\boldsymbol{q}_{0}^{(l)}$ independent (so $\boldsymbol{q}_{0}^{(l+1)}$ independent) part is estimated as

$$
R_{1}+R_{2}+R_{3}+\frac{\partial T_{l}}{\partial t} \sim \frac{\epsilon^{l}}{t^{l+1}}
$$

while $\boldsymbol{q}_{0}^{(l)}$ dependent (so $\boldsymbol{q}_{0}^{(l+1)}$ dependent) part is estimated as

$$
R_{4} \sim \frac{\epsilon^{2(m-1)}}{t^{m}} \frac{\epsilon^{l}}{t^{l}}
$$

The residual part

$$
R=R_{1}+R_{2}+R_{3}+R_{4}+\frac{\partial T_{l}}{\partial t}
$$

is decomposed into non-oscillating part $R_{0}$, slowly oscillating part $R_{s}$ and fast oscillating part $R_{f}$;

$$
R=R_{0}+R_{s}+R_{f}
$$

where

$$
\begin{aligned}
& R_{0}=R_{\mathbf{0}}\left(\boldsymbol{p}^{(l+1)}, t\right) \sim \frac{\epsilon^{l}}{t^{l+1}}, \\
& R_{s}=\sum_{\boldsymbol{k}_{1} \neq \mathbf{0}} R_{\boldsymbol{k}_{1}}\left(\boldsymbol{p}^{(l+1)}, t\right) \exp \left[i \boldsymbol{k}_{1} \cdot \boldsymbol{q}_{1}^{(l+1)}\right] \sim \frac{\epsilon^{l}}{t^{l+1}}, \\
& R_{f}=\sum_{\boldsymbol{k}_{0} \neq \mathbf{0}} R_{\boldsymbol{k}_{0} \boldsymbol{k}_{1}}\left(\boldsymbol{p}^{(l+1)}, t\right) \exp \left[i \boldsymbol{k}_{0} \cdot \boldsymbol{q}_{0}^{(l+1)}+i \boldsymbol{k}_{1} \cdot \boldsymbol{q}_{1}^{(l+1)}\right] \sim \frac{\epsilon^{2(m-1)}}{t^{m}} \frac{\epsilon^{l}}{t^{l}}
\end{aligned}
$$

We define

$$
\begin{aligned}
D_{l+1}\left(\boldsymbol{p}^{(l+1)}, t\right) & =D_{l}\left(\boldsymbol{p}^{(l+1)}, t\right)+R_{0}, \\
E_{l+1}\left(\boldsymbol{q}_{1}^{(l+1)}, \boldsymbol{p}^{(l+1)}, t\right) & =R_{s}, \\
B_{l+1}\left(\boldsymbol{q}^{(l+1)}, \boldsymbol{p}^{(l+1)}, t\right) & =B_{l}\left(\boldsymbol{q}^{(l+1)}, \boldsymbol{p}^{(l+1)}, t\right)+R_{f},
\end{aligned}
$$

By transforming canonically, we succeeded in lowering the residual part $E_{l} \sim \epsilon^{l-1} / t^{l}$ to $E_{l+1} \sim \epsilon^{l} / t^{l+1}$.

\section{$\S$ D Appendix; Proof of Propositions 5.5, 5.6}

\section{D.1 Proof of Proposition 5.5}

\section{Lemma}

$$
\begin{aligned}
\left\|\frac{\delta \boldsymbol{q}_{0}}{t}\right\| & \leq\left|\delta \boldsymbol{q}_{0}(1)\right|+\epsilon\left|\delta \boldsymbol{q}_{1}(1)\right|+\frac{1}{\epsilon^{2}}\left|\delta \boldsymbol{p}_{0}(1)\right|+\frac{1}{\epsilon}\left|\delta \boldsymbol{p}_{1}(1)\right|, \\
\left\|\delta \boldsymbol{p}_{0}\right\| & \leq \epsilon^{4}\left|\delta \boldsymbol{q}_{0}(1)\right|+\epsilon^{4}\left|\delta \boldsymbol{q}_{1}(1)\right|+\left|\delta \boldsymbol{p}_{0}(1)\right|+\epsilon^{3}\left|\delta \boldsymbol{p}_{1}(1)\right| \\
\left\|\frac{\delta \boldsymbol{q}_{1}}{t}\right\| & \leq \epsilon^{3}\left|\delta \boldsymbol{q}_{0}(1)\right|+\left|\delta \boldsymbol{q}_{1}(1)\right|+\frac{1}{\epsilon}\left|\delta \boldsymbol{p}_{0}(1)\right|+\left|\delta \boldsymbol{p}_{1}(1)\right| \\
\left\|\delta \boldsymbol{p}_{1}\right\| & \leq \epsilon^{4}\left|\delta \boldsymbol{q}_{0}(1)\right|+\epsilon^{2}\left|\delta \boldsymbol{q}_{1}(1)\right|+\epsilon\left|\delta \boldsymbol{p}_{0}(1)\right|+\left|\delta \boldsymbol{p}_{1}(1)\right|
\end{aligned}
$$


Proof

From the variational equations of $H^{(3,3)}$, we obtain

$$
\begin{aligned}
\left|\frac{\delta \boldsymbol{q}_{0}}{t}\right| & \leq\left|\delta \boldsymbol{q}_{0}(1)\right|+\frac{1}{\epsilon^{2}}\left\|\delta \boldsymbol{p}_{0}\right\|+\frac{1}{\epsilon}\left\|\delta \boldsymbol{p}_{1}\right\|+\epsilon^{4}\left\|\frac{\delta \boldsymbol{q}_{0}}{t}\right\|+\epsilon^{2}\left\|\frac{\delta \boldsymbol{q}_{1}}{t}\right\|, \\
\left|\delta \boldsymbol{p}_{0}\right| & \leq\left|\delta \boldsymbol{p}_{0}(1)\right|+\epsilon^{4}\left\|\frac{\delta \boldsymbol{q}_{0}}{t}\right\|+\epsilon^{4}\left\|\frac{\delta \boldsymbol{p}_{0}}{t}\right\|+\epsilon^{4}\left\|\frac{\delta \boldsymbol{q}_{1}}{t}\right\|+\epsilon^{4}\left\|\frac{\delta \boldsymbol{p}_{1}}{t}\right\|, \\
\left|\frac{\delta \boldsymbol{q}_{1}}{t}\right| & \leq\left|\delta \boldsymbol{q}_{1}(1)\right|+\frac{1}{\epsilon}\left\|\delta \boldsymbol{p}_{0}\right\|+\left\|\delta \boldsymbol{p}_{1}\right\|+\epsilon^{2}\left\|\frac{\delta \boldsymbol{q}_{1}}{t}\right\|+\epsilon^{4}\left\|\frac{\delta \boldsymbol{q}_{0}}{t}\right\|, \\
\left|\delta \boldsymbol{p}_{1}\right| & \leq\left|\delta \boldsymbol{p}_{1}(1)\right|+\epsilon^{2}\left\|\frac{\delta \boldsymbol{p}_{0}}{t}\right\|+\epsilon^{2}\left\|\frac{\delta \boldsymbol{q}_{1}}{t}\right\|+\epsilon^{2}\left\|\frac{\delta \boldsymbol{p}_{1}}{t}\right\|+\epsilon^{4}\left\|\frac{\delta \boldsymbol{q}_{0}}{t}\right\| .
\end{aligned}
$$

From these inequalities, we can deduce the inequalities of the Lemma by tedious manipulations.

Proof End

We assume that

$$
\left|\delta \boldsymbol{q}_{0}(1)\right| \sim\left|\delta \boldsymbol{p}_{0}(1)\right| \sim\left|\delta \boldsymbol{q}_{1}(1)\right| \sim\left|\delta \boldsymbol{p}_{1}(1)\right| \sim 1
$$

since in the linear perturbation, the scale of the perturbation variables is arbitrary. Then we obtain the proposition below.

\section{Lemma}

$$
\begin{aligned}
\left\|\frac{\delta \boldsymbol{q}_{0}}{t}\right\| & \leq \frac{1}{\epsilon^{2}} \\
\left\|\delta \boldsymbol{p}_{0}\right\| & \leq 1 \\
\left\|\frac{\delta \boldsymbol{q}_{1}}{t}\right\| & \leq \frac{1}{\epsilon} \\
\left\|\delta \boldsymbol{p}_{1}\right\| & \leq 1
\end{aligned}
$$

By using the above lemma to the evolution equations of perturbation variables, we obtain the evaluation of proposition 5.5.

\section{D.2 Proof of Proposition 5.6}

Since $S_{m} \sim \epsilon^{2 m} / t^{m}$, we obtain

$$
\begin{aligned}
\left|\delta \boldsymbol{q}^{(1,1)}-\delta \boldsymbol{q}^{(m, 1)}\right| & \leq \frac{\epsilon^{2}}{t}\left|\delta \boldsymbol{x}^{(m, 1)}\right| \\
\left|\delta \boldsymbol{p}^{(1,1)}-\delta \boldsymbol{p}^{(m, 1)}\right| & \leq \frac{\epsilon^{2}}{t}\left|\delta \boldsymbol{x}^{(m, 1)}\right|
\end{aligned}
$$

where

$$
|\delta \boldsymbol{x}|=|\delta \boldsymbol{q}|+|\delta \boldsymbol{p}|
$$


On the other hand, since $T_{l} \sim \epsilon^{l} / t^{l}$ and $T_{l}$ does not depend on $\boldsymbol{q}_{0}$, we obtain

$$
\begin{aligned}
\left|\delta \boldsymbol{q}^{(m, 1)}-\delta \boldsymbol{q}^{(m, l)}\right| & \leq \frac{\epsilon}{t}\left|\delta \boldsymbol{y}^{(m, l)}\right| \\
\left|\delta \boldsymbol{p}_{0}^{(m, 1)}-\delta \boldsymbol{p}_{0}^{(m, l)}\right| & =0 \\
\left|\delta \boldsymbol{p}_{1}^{(m, 1)}-\delta \boldsymbol{p}_{1}^{(m, l)}\right| & \leq \frac{\epsilon}{t}\left|\delta \boldsymbol{y}^{(m, l)}\right|
\end{aligned}
$$

where

$$
|\delta \boldsymbol{y}|=\left|\delta \boldsymbol{q}_{1}\right|+|\delta \boldsymbol{p}|
$$

By using the above inequalities, we obtain the lemma below.

\section{Lemma}

$$
\begin{aligned}
\left|\delta \boldsymbol{q}^{(1,1)}-\delta \boldsymbol{q}^{(m, l)}\right| & \leq \frac{\epsilon}{t}\left|\delta \boldsymbol{y}^{(m, l)}\right|+\frac{\epsilon^{2}}{t}\left|\delta \boldsymbol{q}_{0}^{(m, l)}\right|, \\
\left|\delta \boldsymbol{p}_{0}^{(1,1)}-\delta \boldsymbol{p}_{0}^{(m, l)}\right| & =\frac{\epsilon^{2}}{t}\left|\delta \boldsymbol{x}^{(m, l)}\right| \\
\left|\delta \boldsymbol{p}_{1}^{(1,1)}-\delta \boldsymbol{p}_{1}^{(m, l)}\right| & \leq \frac{\epsilon}{t}\left|\delta \boldsymbol{y}^{(m, l)}\right|+\frac{\epsilon^{2}}{t}\left|\delta \boldsymbol{q}_{0}^{(m, l)}\right|,
\end{aligned}
$$

Since

$$
\begin{aligned}
\frac{1}{t}\left|\delta \boldsymbol{y}^{(3,3)}\right| & \leq \frac{1}{\epsilon} \\
\frac{1}{t}\left|\delta \boldsymbol{q}_{0}^{(3,3)}\right| & \leq \frac{1}{\epsilon^{2}} \\
\frac{1}{t}\left|\delta \boldsymbol{x}^{(3,3)}\right| & \leq \frac{1}{\epsilon^{2}}
\end{aligned}
$$

we obtain the proposition 5.6.

\section{$\S \mathrm{E} \quad$ Growth Index of Perturbations}

In this appendix, we calculate the growth rates of perturbations in the first model $\left(\lambda \phi_{1}^{2} \phi_{2}\right.$, $\left.2 \mu_{1} \approx \mu_{2}\right)$ and the second model $\left(\lambda \phi_{1}^{2} \phi_{2}^{2}, \mu_{1} \approx \mu_{2}\right)$ which are presented in the beginning of $\S 4$, assuming that $\lambda / \epsilon$ is of order unity.

The fourth order Hamiltonians $H^{(4)}$ of the first model and the second model are written by

$$
\begin{aligned}
H & =\frac{2}{\epsilon}(\boldsymbol{\omega} \cdot \boldsymbol{P})^{1 / 2}+A+R \\
A & =\frac{\eta}{\epsilon} \frac{1}{t^{\gamma}} \frac{1}{(\boldsymbol{\omega} \cdot \boldsymbol{P})^{1 / 2}}\left(M+N \cos k Q_{1}\right), \\
|R| & \leq \frac{\epsilon}{t^{2}}
\end{aligned}
$$

where

$$
\begin{aligned}
& \gamma=1 \quad k=1 \\
& M=0 \quad N=\left(P_{0}-2 P_{1}\right) P_{1}^{1 / 2}
\end{aligned}
$$


and

$$
\begin{aligned}
& \gamma=2 \quad k=2 \\
& M=2 N=\left(P_{0}-2 P_{1}\right) P_{1},
\end{aligned}
$$

respectively. The coefficients of the perturbation equations

$$
\begin{aligned}
\frac{d}{d t}\left(\begin{array}{c}
\delta Q_{1} \\
\delta P_{1}
\end{array}\right)= & \left(\begin{array}{cc}
\frac{\partial^{2} A}{\partial P_{1} \partial Q_{1}} & -\frac{1}{2} \frac{\omega_{1}^{2}}{\epsilon} \frac{1}{(\boldsymbol{\omega} \cdot \boldsymbol{P})^{3 / 2}}+\frac{\partial^{2} A}{\partial P_{1}^{2}} \\
-\frac{\partial^{2} A}{\partial Q_{1}^{2}} & -\frac{\partial^{2} A}{\partial Q_{1} \partial P_{1}}
\end{array}\right)\left(\begin{array}{c}
\delta Q_{1} \\
\delta P_{1}
\end{array}\right) \\
& +\left(\begin{array}{cc}
-\frac{1}{2} \frac{\omega_{0} \omega_{1}}{\epsilon} \frac{1}{(\boldsymbol{\omega} \cdot \boldsymbol{P})^{3 / 2}}+\frac{\partial^{2} A}{\partial P_{1} \partial P_{0}}
\end{array}\right) \delta P_{0}+\boldsymbol{R}, \\
\frac{d}{d t} \delta Q_{0}= & \left(-\frac{\partial^{2} A}{\partial Q_{1} \partial P_{0}} \frac{\omega_{0}^{2}}{\epsilon} \frac{1}{(\boldsymbol{\omega} \cdot \boldsymbol{P})^{3 / 2}}+\frac{\partial^{2} A}{\partial P_{0}^{2}}\right) \delta P_{0}+\frac{\partial^{2} A}{\partial P_{0} \partial Q_{1}} \delta Q_{1} \\
& +\left(-\frac{1}{2} \frac{\omega_{0} \omega_{1}}{\epsilon} \frac{1}{(\boldsymbol{\omega} \cdot \boldsymbol{P})^{3 / 2}}+\frac{\partial^{2} A}{\partial P_{0} \partial P_{1}}\right) \delta P_{1}+R, \\
\delta P_{0}= & \delta P_{0}(1), \\
|R| \leq & \frac{\epsilon}{t^{2}}\left(\left|\delta P_{0}\right|+\left|\delta Q_{1}\right|+\left|\delta P_{1}\right|\right),
\end{aligned}
$$

are given by

$$
\begin{aligned}
\left(\frac{\eta}{\epsilon} \frac{1}{t^{\gamma}}\right)^{-1} \frac{\partial^{2} A}{\partial P_{1} \partial Q_{1}} & =-k \frac{1}{(\boldsymbol{\omega} \cdot \boldsymbol{P})^{1 / 2}} \frac{\partial N}{\partial P_{1}} \sin k Q_{1}+\frac{1}{2} k \frac{\omega_{1}}{(\boldsymbol{\omega} \cdot \boldsymbol{P})^{3 / 2}} N \sin k Q_{1}, \quad \text { (E.14) } \\
\left(\frac{\eta}{\epsilon} \frac{1}{t^{\gamma}}\right)^{-1} \frac{\partial^{2} A}{\partial P_{1}^{2}}= & \frac{3}{4} \frac{\omega_{1}^{2}}{(\boldsymbol{\omega} \cdot \boldsymbol{P})^{5 / 2}}\left(M+N \cos k Q_{1}\right)-\frac{\omega_{1}}{(\boldsymbol{\omega} \cdot \boldsymbol{P})^{3 / 2}}\left(\frac{\partial M}{\partial P_{1}}+\frac{\partial N}{\partial P_{1}} \cos k Q_{1}\right) \\
+ & \frac{1}{(\boldsymbol{\omega} \cdot \boldsymbol{P})^{1 / 2}}\left(\frac{\partial^{2} M}{\partial P_{1}^{2}}+\frac{\partial^{2} N}{\partial P_{1}^{2}} \cos k Q_{1}\right), \\
\left(\frac{\eta}{\epsilon} \frac{1}{t^{\gamma}}\right)^{-1} \frac{\partial^{2} A}{\partial P_{1} \partial P_{0}}= & \frac{3}{4} \frac{\omega_{0} \omega_{1}}{(\boldsymbol{\omega} \cdot \boldsymbol{P})^{5 / 2}}\left(M+N \cos k Q_{1}\right)-\frac{1}{2} \frac{\omega_{0}}{(\boldsymbol{\omega} \cdot \boldsymbol{P})^{3 / 2}}\left(\frac{\partial M}{\partial P_{1}}+\frac{\partial N}{\partial P_{1}} \cos k Q_{1}\right) \\
& -\frac{1}{2} \frac{\omega_{1}}{(\boldsymbol{\omega} \cdot \boldsymbol{P})^{3 / 2}}\left(\frac{\partial M}{\partial P_{0}}+\frac{\partial N}{\partial P_{0}} \cos k Q_{1}\right) \\
& +\frac{1}{(\boldsymbol{\omega} \cdot \boldsymbol{P})^{1 / 2}}\left(\frac{\partial^{2} M}{\partial P_{1} \partial P_{0}}+\frac{\partial^{2} N}{\partial P_{1} \partial P_{0}} \cos k Q_{1}\right),
\end{aligned}
$$




$$
\begin{gathered}
\left(\frac{\eta}{\epsilon} \frac{1}{t^{\gamma}}\right)^{-1} \frac{\partial^{2} A}{\partial Q_{1}^{2}}=-k^{2} \frac{1}{(\boldsymbol{\omega} \cdot \boldsymbol{P})^{1 / 2}} N \cos k Q_{1}, \\
\left(\frac{\eta}{\epsilon} \frac{1}{t^{\gamma}}\right)^{-1} \frac{\partial^{2} A}{\partial Q_{1} \partial P_{0}}=\frac{1}{2} k \frac{\omega_{0}}{(\boldsymbol{\omega} \cdot \boldsymbol{P})^{3 / 2}} N \sin k Q_{1}-k \frac{1}{(\boldsymbol{\omega} \cdot \boldsymbol{P})^{1 / 2}} \frac{\partial N}{\partial P_{0}} \sin k Q_{1},
\end{gathered}
$$

and

$$
\begin{aligned}
\left(\frac{\eta}{\epsilon} \frac{1}{t^{\gamma}}\right)^{-1} \frac{\partial^{2} A}{\partial P_{0}^{2}}= & \frac{3}{4} \frac{\omega_{0}^{2}}{(\boldsymbol{\omega} \cdot \boldsymbol{P})^{5 / 2}}\left(M+N \cos k Q_{1}\right)-\frac{\omega_{0}}{(\boldsymbol{\omega} \cdot \boldsymbol{P})^{3 / 2}}\left(\frac{\partial M}{\partial P_{0}}+\frac{\partial N}{\partial P_{0}} \cos k Q_{1}\right) \\
& +\frac{1}{(\boldsymbol{\omega} \cdot \boldsymbol{P})^{1 / 2}}\left(\frac{\partial^{2} M}{\partial P_{0}^{2}}+\frac{\partial^{2} N}{\partial P_{0}^{2}} \cos k Q_{1}\right) .
\end{aligned}
$$

Since the correction to the growth rate of perturbations by $R$ is of order $\epsilon$ as long as we consider the finite small time range, we consider the fixed points with dropping $R$.

For simplicity, we consider the case $\omega_{1}=0$.

In the first model, around the fixed point

$$
Q_{1}=\frac{\pi}{2}+k \pi \quad 2 P_{1}=P_{0}=c
$$

where $k$ is integer, the perturbations are given by

$$
\begin{aligned}
\delta Q_{1}= & t^{(-)^{k} \Gamma_{1}} \delta Q_{1}(1) \\
\delta P_{1}= & t^{(-)^{k+1} \Gamma_{1}}\left(\delta P_{1}(1)+\frac{1}{2} \delta P_{0}(1)\right)-\frac{1}{2} \delta P_{0}(1), \\
\delta Q_{0}= & \delta Q_{0}(1)-\frac{1}{2 \epsilon} \frac{\omega_{0}^{1 / 2}}{c^{3 / 2}} \delta P_{0}(1)(t-1) \\
& -\frac{1}{2}\left(t^{(-)^{k} \Gamma_{1}}-1\right) \delta Q_{1}(1), \\
\delta P_{0}= & \delta P_{0}(1),
\end{aligned}
$$

where $\Gamma_{1}$ is given by

$$
\Gamma_{1}=\sqrt{2} \frac{\eta}{\epsilon} \frac{1}{\omega_{0}^{1 / 2}}
$$

The lines $Q_{1}=\pi / 2+k \pi$ and $P_{1}=c / 2$ are the heteroclinic orbits. Since the definition of the Bardeen parameter $\zeta$ contains the prefactor $1 / t$, when $\Gamma_{1}$ is larger than $1, \zeta$ grows in proportion to $t^{\Gamma_{1}-1}$. Around the elliptic fixed point

$$
Q_{1}=k \pi \quad 6 P_{1}=P_{0}=c,
$$

the evolution of perturbations is oscillatory.

In the second model, the fixed points are given by

$$
Q_{1}=\frac{k}{2} \pi \quad 2 P_{1}=P_{0}=c,
$$


where for odd $k$ hyperbolic, for even $k$ elliptic. Around this hyperbolic fixed point the perturbations are given by

$$
\begin{aligned}
\left(\begin{array}{c}
\delta Q_{1} \\
\delta P_{1}
\end{array}\right)= & c_{1} \exp \left[\Gamma_{2}\left(1-\frac{1}{t}\right)\right]\left(\begin{array}{c}
1 \\
-\frac{\sqrt{2}}{2} c
\end{array}\right) \\
& +c_{2} \exp \left[-\Gamma_{2}\left(1-\frac{1}{t}\right)\right]\left(\begin{array}{c}
1 \\
\frac{\sqrt{2}}{2} c
\end{array}\right)+\delta P_{0}(1)\left(\begin{array}{c}
0 \\
\frac{1}{2}
\end{array}\right)
\end{aligned}
$$

where

$$
\begin{aligned}
c_{1} & =\frac{1}{2} \delta Q_{1}(1)-\frac{\sqrt{2}}{2} \frac{1}{c} \delta P_{1}(1)+\frac{\sqrt{2}}{4} \frac{1}{c} \delta P_{0}(1), \\
c_{2} & =\frac{1}{2} \delta Q_{1}(1)+\frac{\sqrt{2}}{2} \frac{1}{c} \delta P_{1}(1)-\frac{\sqrt{2}}{4} \frac{1}{c} \delta P_{0}(1),
\end{aligned}
$$

and

$$
\begin{aligned}
\delta Q_{0}= & \delta Q_{0}(1)-\frac{1}{2} \frac{1}{\epsilon} \frac{\omega_{0}^{1 / 2}}{c^{3 / 2}} \delta P_{0}(1)(t-1) \\
& -\frac{5}{32} \frac{\eta}{\epsilon} \frac{1}{\left(\omega_{0} c\right)^{1 / 2}} \delta P_{0}(1)\left(1-\frac{1}{t}\right)+R \\
\delta P_{0}= & \delta P_{0}(1)
\end{aligned}
$$

where

$$
|R| \leq \frac{\eta}{\epsilon}\left\|\delta P_{1}\right\|
$$

The growth rate $\Gamma_{2}$ is given by

$$
\Gamma_{2}=\frac{\sqrt{2}}{2} \frac{\eta}{\epsilon} \frac{c^{1 / 2}}{\omega_{0}^{1 / 2}} .
$$

The contribution of the $\eta$ dependent part to the Bardeen parameter $\zeta$ is proportional to $f(t)$ :

$$
f(t)=\frac{1}{t} \exp \left[\Gamma_{2}\left(1-\frac{1}{t}\right)\right]
$$

which increases for $t \leq \Gamma_{2}$ and

$$
\frac{f\left(\Gamma_{2}\right)}{f(1)}=\frac{1}{\Gamma_{2}} \exp \left(\Gamma_{2}-1\right)
$$

In case $\omega_{1} \neq 0$, as the time proceeds the term originating from the unperturbed part becomes dominant while the perturbation parts decay as $1 / t^{\gamma}$, therefore the fixed points disappear.

\section{References}

[1] Kodama, H. and Sasaki, M., Prog. Theor. Phys. Suppl. 78, 1-166 (1984). 
[2] Bardeen, J.M., Phys. Rev. D 22, 1882 (1980).

[3] Taruya, A. and Nambu, Y., Phys. Lett. B428 37-43 (1998)

[4] Kodama, H. and Hamazaki, T., Phys. Rev. D57, 7177-7185 (1998).

[5] Sasaki, M. and Tanaka, T, Prog. Theor. Phys. 99,763-782 (1998)

[6] Kodama, H. and Hamazaki, T., Prog. Theor. Phys. 96,949-970 (1996)

[7] Hamazaki, T. and Kodama, H., Prog. Theor. Phys. 96,1123-1146 (1996)

[8] Hamazaki, T., Phys. Rev. D 66, 023529 (2002)

[9] Traschen, J. and Brandenberger, R.H. Phys. Rev. D42, 2491 (1990); Shtanov, Y., Traschen, J. and Brandenberger, R.H. Phys. Rev. D51, 5438 (1995); Kofman, K.A., Linde, A.D. and Starobinsky, A.A. Phys. Rev. Lett.73, 3195 (1994); Kofman, K.A., Linde, A.D. and Starobinsky, A.A. Phys. Rev. D56, 3258 (1997)

[10] Khlebnikov, S.Yu. and Tkachev, I.I. Phys. Rev. Lett.77, 219 (1996)

[11] Gordon, C., Wands, D., Bassett, B.A. and Maartens, R., Phys. Rev D63 123506 (2001)

[12] Linde, A.D., Phys. Lett. B158, 375 (1985); Polarski, D. and Starobinsky, A.A., Nucl. Phys. B385 623 (1992); Starobinski, A.A. and Yokoyama, J, gr-qc/9502002 Linde, A.D. and Kofman, K.A., Nucl. Phys. B282, 555 (1987); Liddle, A.R. and Mazumdar, A., Phys. Rev. D61, 123507 (2000)

[13] Zibin, J.P., Brandenberger, R and Scott, D, Phys. Rev. D63, 043511 (2001)

[14] Felder, G and Kofman, L, Phys. Rev. D63, 103503 (2001)

[15] Arnold, V.I., Mathematical Methods of Classical Mechanics, (Springer, New York) (1978); Arnold, V.I. and Avez, A. Problèmes ergodiques de la mécanique classique, (Ganthier-Villars, Paris) (1967) 